\title{
Mikro-Ionenstrahl-Apparatur zur Exposition lebender Zellen
}

\author{
Dissertation \\ zur Erlangung des Doktorgrades \\ der Mathematisch-Naturwissenschaftlichen Fakultäten \\ der Georg-August-Universität zu Göttingen
}

vorgelegt von

Klaus-Dieter Greif

aus Dinslaken

Göttingen 2002 
D7

Referent: $\quad$ Prof. Dr. G. Schmahl

Koreferent: $\quad$ Prof. Dr. D. Frankenberg

Tag der mündlichen Prüfung: 


\section{Inhaltsverzeichnis}

$\begin{array}{llc}1 & \text { Einleitung } & 6\end{array}$

1.1 Größen und Begriffe im Strahlenschutz und in der Strahlenbiologie 7

1.2 Motivation und Ziel . . . . . . . . . . . . . . . . . . 12

1.3 Die Ionenbeschleunigeranlage der PTB . . . . . . . . . . . 15

1.4 Prinzipieller Aufbau des Mikro-Ionenstrahls . . . . . . . . . . 17

2 Grundlagen der Ionenoptik 22

2.1 Koordinatensystem . . . . . . . . . . . . . . 22

2.2 Bahngleichungen . . . . . . . . . . . . . . . . 23

2.3 Magnetfelder in der Strahloptik . . . . . . . . . . . . . . . 25

2.4 Allgemeine Lösung in 1. Ordnung . . . . . . . . . . . . . . 26

2.5 Matrixschreibweise . . . . . . . . . . . . . 27

2.6 Teilchenstrahl und Emittanz . . . . . . . . . . . . . . 28

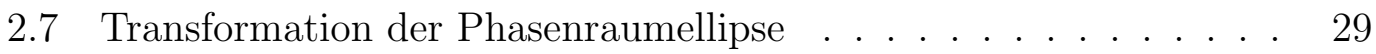

2.7.1 Dispersive Aufweitung der Phasenraumellipse . . . . . . 30

2.8 Transfermatrizen ausgewählter Elemente . . . . . . . . . . . . 31

2.8 .1 Driftstrecke . . . . . . . . . . . . . . 31

2.8.2 Quadrupolmagnet.................. 32

2.8.3 Analysiersystem . . . . . . . . . . . . . . 33

2.9 Transfermatrix 2. Ordnung . . . . . . . . . . . . . . . 35 
3 Optik des Mikrostrahls $\quad 36$

3.1 Abbildung in 1. Ordnung . . . . . . . . . . . . . . 36

3.2 Intrinsische Aberrationen . . . . . . . . . . . . . . . . 37

3.3 Parasitäre Aberrationen . . . . . . . . . . . . . . . . 39

3.4 Berechnung der Abbildungseigenschaften . . . . . . . . . . . 42

3.4.1 Kompensation der Dispersion ............ . 43

3.4.2 Optische Parameter des Mikro-Ionenstrahls . . . . . . . . . 45

4 Elemente der Strahlführung $\quad 48$

4.1 Objekt- und Aperturblende . . . . . . . . . . . . . . 48

4.2 Deflektor . . . . . . . . . . . . . . . . . 51

4.3 Magnete .......................... 55

4.3 .1 Dipolmagnet . . . . . . . . . . . . . . 55

4.3.2 Quadrupolmagnete ................ 56

4.4 Schlitzstrommessung . . . . . . . . . . . . . . . 57

$\begin{array}{lll}5 & \text { Experimentierplatz } & 60\end{array}$

5.1 Strahldiagnose mit Szintillator und CCD-Kamera . . . . . . . 61

5.1 .1 Einfluss der Schärfentiefe . . . . . . . . . . . . . 62

5.2 Detektion einzelner Ionen . . . . . . . . . . . . 63

5.3 Streuung der Ionen nach dem Austritt aus dem Vakuum . . . . . 67

5.4 Fenstermechanik . . . . . . . . . . . . . . . 69

5.5 Ablaufsteuerung . . . . . . . . . . . . . . . 71

5.6 Zellerkennung und Zellpositionierung . . . . . . . . . . . . . 72

5.6.1 Genauigkeit des Mikropositioniertisches . . . . . . . . . 76 
$\begin{array}{lll}6 & \text { Messungen der Strahlparameter } & 79\end{array}$

6.1 Fokussierung . . . . . . . . . . . . . . . . . . . . . . . . 79

6.1 .1 Justierung des Folienstrahls . . . . . . . . . . . . . . . . . 79

6.1.2 Optimierung des Mikrostrahls ............ 80

6.1.3 Auswirkungen von parasitären Aberrationen . . . . . . . . 82

6.2 Teilchendetektion . . . . . . . . . . . . . . . . . 84

6.2 .1 Spurdetektor . . . . . . . . . . . . . . . 84

6.2.2 Silizium-Halbleiterzähler . . . . . . . . . . . . . . 85

6.2.3 Szintillator und Photomultiplier . . . . . . . . . . 87

6.3 Ortsauflösung . . . . . . . . . . . . . . . . . . 91

6.4 Irrläufer . . . . . . . . . . . . . . . . . . . . 94

$\begin{array}{lll}7 & \text { Zusammenfassung und Ausblick } & 97\end{array}$

7.1 Status der Anlage . . . . . . . . . . . . . . . . . . 97

7.2 Apparative Verbesserungen . . . . . . . . . . . . . . . . . . . 98

7.3 Weitere Anwendungsgebiete . . . . . . . . . . . . 100

$\begin{array}{lr}\text { Danksagung } & 109\end{array}$ 


\section{Kapitel 1}

\section{Einleitung}

Die Entstehung von Krebs ist ein komplexer und mehrstufiger Prozess, und erst nach und nach beginnt die Wissenschaft, seine Details zu verstehen. Es gilt aber als sicher, dass Tumoren aus einer einzigen bösartig transformierten Zelle hervorgehen. Die Transformation wird durch mehrere spezifische Änderungen der Erbinformation verursacht, bei denen krebsverhindernde Gene (Tumorsuppressorgene) durch Mutation inaktiviert werden und aus bestimmten anderen Genen krebsauslösende Gene, sogenannte Onkogene, entstehen. Als auslösende Agenzien für diese Mutationen kommen chemische, meist oxidativ wirkende Substanzen oder Viren, aber auch ionisierende Strahlung in Frage. Epidemiologische Studien sowie Tierversuche haben die karzinogene Wirkung vieler Agenzien, auch von ionisierender Strahlung, nachgewiesen.

Im Fall der ionisierenden Strahlung kann deren Wirkung durch die Bestrahlung von lebenden Zellen in vitro untersucht und quantifiziert werden. Die Quantifizierung geschieht mittels verschiedener biologischer Endpunkte, die ein Maß für die Wirkung der ionisierenden Strahlen bieten können. Anhand solcher Messungen können Dosis-Wirkungs-Beziehungen auf zellulärer Ebene ermittelt werden.

Neuere Untersuchungen haben gezeigt, dass das Verhalten der Zellen eine wichtige Rolle bei der Wirkung ionisierender Strahlung spielt. Zellen setzen bei einer Bestrahlung eine Reihe von Mechanismen in Gang, u. a. die enzymatische Reparatur von DNA-Schäden, die Variation des eigenen Zellzyklus oder interzelluläre Kommunikation. Diese für die Karzinogenese möglicherweise bedeutsamen Effekte sind noch nicht im Detail verstanden.

Zu ihrer Untersuchung ist es notwendig, die verschiedenen Parameter einer in vitro Bestrahlung möglichst genau zu kontrollieren und einzustellen. Bei herkömmlicher Bestrahlung können nur statistische Erwartungswerte der Bestrahlungsparameter eingestellt werden, so dass eine Zuordnung von primären Bestrahlungsereignissen und erzielter Wirkung nur eingeschränkt möglich ist. Wenn es 
bei einer Bestrahlung mit Ionen gelingt, die Anzahl, die Energie, die genaue Position und den Trefferzeitpunkt der Ionen zu kontrollieren, kann ein Teil der stochastischen Natur der primären Bestrahlungsereignisse ausgeschaltet werden. Ein besseres Verständnis der zellulären und molekularen Effekte ionisierender Strahlung wird dann möglich.

Ziel dieser Arbeit ist es daher, eine Apparatur aufzubauen, die es ermöglicht, lebende Zellen mit einer wohldefinierten Anzahl von Ionen mit einer Ortsaufösung im subzellularen Bereich zu bestrahlen. Diese wird im Folgenden als MikroIonenstrahl bezeichnet.

\subsection{Größen und Begriffe im Strahlenschutz und in der Strahlenbiologie}

Für die Quantifizierung und das Verständnis der Effekte ionisierender Strahlung sind detaillierte Kenntnisse der Wechselwirkungsmechanismen von Strahlung mit Materie unabdingbare Voraussetzung. Im Folgenden sollen einige grundlegende Begriffe eingeführt werden.

Ionisierende Strahlung kann durch eine Vielzahl von Größen beschrieben werden, wie z.B. Teilchenfluenz, Ionendosis, Energiedosis oder Äquivalentdosis. Das Konzept der Äquivalentdosis $H$ wurde 1980 eingeführt [1]. Im Gegensatz zu anderen Dosiseinheiten, die allein auf physikalischen Effekten beruhen, berücksichtigt die Äquivalentdosis die Wirksamkeit der verschiedenen Arten ionisierender Strahlung auf lebende Organismen. Sie ist gegeben durch die Energiedeposition ionisierender Strahlung pro Masseelement, auch Energiedosis $D$ genannt, multipliziert mit einem Qualitätsfaktor $Q$, der die unterschiedliche biologische Wirksamkeit verschiedener Strahlenarten und -energien beschreibt [2].1]

$$
H=Q D=Q \frac{d E}{d m}
$$

Die Einheit der Äquivalentdosis ist das Sievert (Sv), wodurch sie von der Energiedosis, deren Einheit das Gray (Gy) ist, unterschieden werden kann. Für den

\footnotetext{
${ }^{1}$ Hier ist die allgemeine Definition der Äquivalentdosis angegeben. Die neuen Dosisgrößen im Strahlenschutz sind aus dieser Definition unter detaillierter Spezifizierung der Randbedingungen abgeleitet.
} 
Qualitätsfaktor ergibt sich somit die Einheit Sv/Gy. Die Äquivalentdosis setzt sich aus einer physikalischen Messgröße, der Energiedosis, und einem biologischen Anteil, der einer direkten Messung nicht zugänglich ist, zusammen. In der radiobiologischen Forschung wird in der Regel die Energiedosis verwendet. Aus dem Vergleich der Wirkungen von Energiedosen verschiedener Strahlenarten werden die Qualitätsfaktoren abgeleitet, welche die Definition der Äquivalentdosis erst erlauben.

Die Energiedosis ist für verschiedene Strahlenarten mit Primärmessinstrumenten und Referenzfeldern mit einer relativen Unsicherheit zwischen 0,1\% und $5 \%$ bestimmbar. Eine Ausnahme bildet die Neutronenstrahlung, bei der die Messunsicherheit je nach Energiebereich zwischen $2 \%$ und $18 \%$ beträgt 2 . Das heißt, der Term $\frac{d E}{d m}$ aus Gleichung 1.1 ist in der Regel mit hoher Genauigkeit bestimmbar.

Der Qualitätsfaktor $Q$ beinhaltet, dass bei gleichem Energieübertrag verschieden starke Wirkungen in biologischen Systemen hervorgerufen werden können. Zur Festlegung des Qualitätsfaktors für eine bestimmte Strahlung muss deren Wirksamkeit in Bezug auf die für den Strahlenschutz relevanten Strahlenschäden bestimmt werden. Diese kann anhand epidemiologischen Studien und mit Modellrechnungen allerdings nur abgeschätzt werden.

Um die Wirkung verschiedener Arten ionisierender Strahlung in Experimenten (in vitro oder in vivo) zu vergleichen, wird der Begriff Relative Biologische Wirksamkeit RBW verwendet (engl.: RBE). Die RBW gibt das Verhältnis von zwei Energiedosen verschiedener Strahlung an, die eine gleich starke Reaktion hervorrufen [2]. Als Maß für die Wirkung können mehrere biologische Messgrößen ${ }^{3}$ verwendet werden. Hierzu gehören u. a. Zellinaktivierung, diverse Chromosomenaberrationen, Mutationen, neoplastische Zelltransformation, DNAFragmentierung oder die Veränderung der Expression bestimmter Gene. Als Referenzstrahlung wird Röntgen- oder Gammastrahlung genommen, deren RBW wird somit zu 1 gesetzt.

$$
R B W=\frac{D_{r e f}}{D}
$$

Es ist zu beachten, dass man für eine bestimmte Strahlung keineswegs einen einzigen RBW-Wert angeben kann. Vielmehr kommen für verschiedene biologische

\footnotetext{
${ }^{2}$ Die Spezifikationen verschiedener Kalibrierfelder sind im Internet unter www.ptb.de veröffentlicht.

${ }^{3}$ Der Auswerteparameter in radiobiologischen Experimenten wird oftmals als Endpunkt bezeichnet.
} 
Endpunkte verschiedene RBW mit erheblichen Abweichungen bei ein und derselben Strahlung heraus [2]. Zudem ist die RBW auch von der Dosis und der Dosisleistung abhängig und insbesondere im Bereich kleiner Dosen mit erheblichen Unsicherheiten behaftet. Die Inhomogenität gemessener RBW-Werte spiegelt die Komplexität und die Individualität biologischer Systeme wider.

Ein weiterer Parameter, der zur Charakterisierung verschiedener Strahlenarten herangezogen wird, ist das Lineare Energieübertragungsvermögen LET (engl.: Linear Energy Transfer). Das LET bezeichnet den Energietransfer entlang eines kurzen Abschnittes der Teilchenspur in Materie [3]. Es ist eine wichtige Kenngröße, da die räumliche Verteilung der Energiedeposition einen wesentlichen Einfluss auf die Wirkung der Strahlung hat. Für verschiedene Strahlenarten variiert das LET erheblich, da dem Energietransfer verschiedene physikalische Effekte zugrunde liegen. Geladene Teilchen geben ihre Energie zumeist durch Ionisation des durchquerten Materials ab. Die hierbei freigesetzten Elektronen entstehen nahe der Teilchenbahn und haben eine statistische Energieverteilung (Sekundärteilchenspektrum), die je nach Teilchenenergie variiert. Die Wechselwirkung von $\gamma$-Strahlung dagegen kann Photoeffekt, Comptoneffekt und Paarbildung beinhalten. Je nach Energie eines $\gamma$-Quants dominiert ein anderer Mechanismus. Neutronen verlieren ihre Energie durch elastische Stöße an Atomkernen (Moderation) und erzeugen so schwere Sekundärteilchen [4].

Man kann das LET eines Teilchens auf verschiedene Arten definieren, je nachdem welche Sekundärelektronenenergien mit berücksichtigt werden sollen. Man bezeichnet dies als beschränktes LET und kennzeichnet es durch den Großbuchstaben L und die tiefgestellte Grenzenergie. $\mathrm{L}_{100}$ bedeutet z.B., dass Sekundärelektronen mit Energien über $100 \mathrm{eV}$ ( $\delta$-Teilchen) nicht berücksichtigt werden, da sie eine hohe Reichweite haben und ihre Energie im wesentlichen fernab der primären Teilchenbahn abgeben. Untersuchungen haben gezeigt, dass das beschränkte Energieübertragungsvermögen $\mathrm{L}_{100}$ ein geeigneter Parameter ist, um die Strahlenqualität zu charakterisieren [5].

Es zeigt sich, dass Strahlung mit einem niedrigen LET $(\leq 10 \mathrm{keV} / \mu \mathrm{m})$ eine RBW nahe eins hat, Hoch-LET-Strahlung $(50 \mathrm{keV} / \mu \mathrm{m}-200 \mathrm{keV} / \mu \mathrm{m})$ die höchsten RBW-Werte hat und die RBW bei sehr hohem LET $(\geq 200 \mathrm{keV} / \mu \mathrm{m})$ wieder abnimmt. Die Zunahme der RBW mit dem LET ist verständlich, da die Dosis bei hohem LET räumlich inhomogen verteilt ist und lokal hohe Dosen auftreten. Die Abnahme bei sehr hohem LET beruht darauf, dass die biologischen Endpunkte 


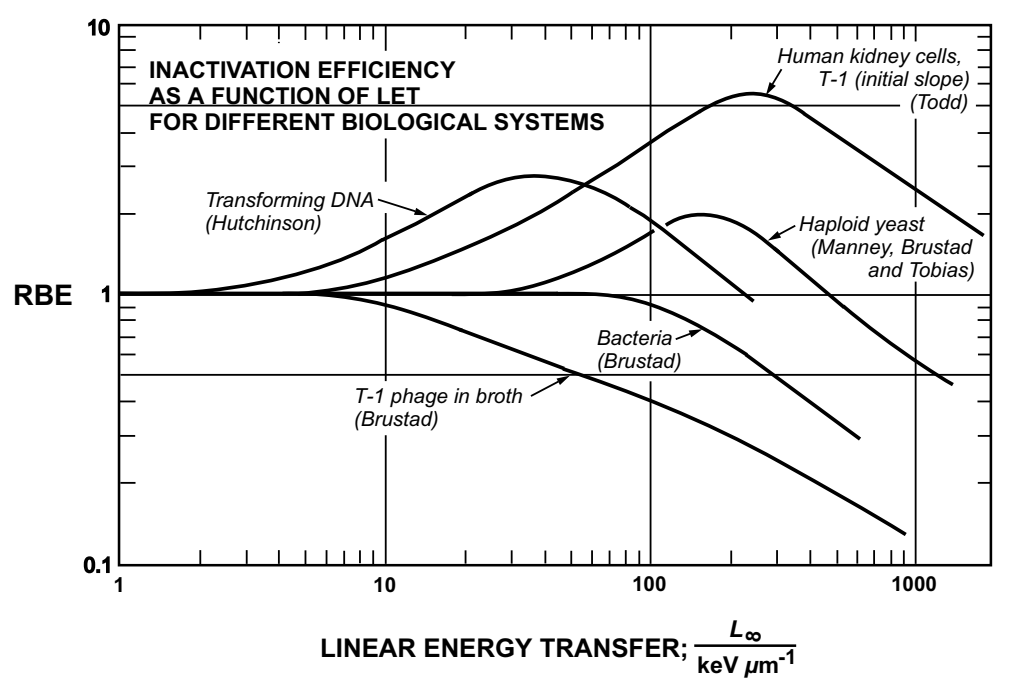

Abbildung 1.1: LET-Abhängigkeit der Relativen Biologischen Wirksamkeit [3].

oft einen Sättigungs- oder Maximalwert erreichen und eine Steigerung der lokalen Energiedeposition die Wirkung nicht erhöhen kann. In Abbildung 1.1 sind einige LET-RBW-Kurven dargestellt. Bis auf eine Kurve handelt es sich um den gleichen Endpunkt (Zellinaktivierung) für verschiedene Zelltypen.

In der seit August 2001 gültigen Strahlenschutzverordnung wurden die Strahlungswichtungsfaktoren ${ }^{4}$ festgelegt, die in Tabelle 1.1 wiedergegeben sind. Diese Werte spiegeln in etwa die mittleren RBW-Werte der aufgelisteten Strahlenarten wider, die in einer Vielzahl radiobiologischer Experimente gewonnen wurden. Die Festlegung dieser Werte erfolgte auf Empfehlung der Internationalen Strahlenschutzkommission ICRP 5 .

Ein weiterer wichtiger Begriff im Strahlenschutz ist der Grenzwert. Die Festlegung der gesetzlich zulässigen Grenzwerte erfolgt durch nationale oder internationale Fachgremien. Eine wichtige Entscheidungsgrundlage hierzu bilden epidemiologische Studien, die sich mit den Überlebenden der Atombombenabwürfe in Hiroshima und Nagasaki [7] sowie Bergleuten aus dem Uranabbau [8] beschäftigen. Die Dosen, die diese Personengruppen erhalten haben, liegen jedoch in der Regel weit über dem Niveau, das für den Strahlenschutz und die Umweltüberwachung

\footnotetext{
${ }^{4}$ Die Strahlungswichtungsfaktoren stellen eine Vereinfachung der Qualitätsfaktoren für den praktischen Strahlenschutz dar.

${ }^{5}$ International Comission on Radiation Protection.
} 


\begin{tabular}{|l|r|}
\hline Strahlung & $w_{R}$ \\
\hline \hline Photonen & 1 \\
\hline Elektronen & 1 \\
\hline Protonen & 5 \\
\hline Alpha-Teilchen & 20 \\
\hline Schwere Ionen \& Spaltfragmente & 20 \\
\hline Neutronen (je nach Energie) & $5-20$ \\
\hline
\end{tabular}

Tabelle 1.1: Strahlungswichtungsfaktoren $w_{R}$ für verschiedene Strahlenarten [6].

relevant ist. Zudem waren die Atombombenopfer im Gegensatz zu beruflich strahlenexponierten Personen einer hohen Dosisleistung ausgesetzt. Die Aussagekraft dieser Daten für den Bereich geringer Dosen und Dosisleistungen ist daher begrenzt [9]. Zudem sind die Dosen, welche anhand von Messungen und Modellen nachträglich rekonstruiert werden, mit erheblichen Unsicherheiten behaftet [10].

Die Schädigung eines Organismus durch ionisierende Strahlung wird in zwei Arten aufgeteilt: stochastische und deterministische Schäden. Deterministische Schäden treten bei hohen Energiedosen (ab ca. 0,5 Gy) und mit hoher Vorhersagbarkeit ein. Sie beinhalten u. a. Hautverbrennungen, Haarausfall und Augenlinsentrübung. Deterministische Schäden können als Nebenwirkung in der medizinischen Strahlentherapie oder bei Unfällen im Umgang mit ionisierender Strahlung auftreten. Als stochastische Strahlenschäden werden solche bezeichnet, die auch bei geringen Dosen mit einer gewissen Wahrscheinlichkeit auftreten können. Strahleninduzierte Karzinogenese und Mutationen in der Keimbahn sind die relevanten stochastischen Strahlenschäden. Die zulässigen Dosisgrenzwerte liegen weit unterhalb des Bereiches, bei dem deterministische Schäden auftreten. Bei der Festlegung von Grenzwerten im Strahlenschutz hat man es daher mit der Risikoabschätzung der stochastischen Schäden zu tun.

Die Strahlenexposition der Bevölkerung liegt in Deutschland im Mittelwert bei ca. $4 \mathrm{mSv}$ pro Jahr, wobei ca. $60 \%$ aus natürlichen und $40 \%$ aus zivilisatorischen Quellen stammt [11]. Medizinische Anwendungen und Inhalation von Radon und Radonfolgeprodukten stellen mit jeweils ca. $1,5 \mathrm{mSv}$ die größten Einzelposten dar. Die Belastung hängt allerdings stark von der Höhe über dem Meeresspiegel (kosmische Strahlung) und dem geologischen Untergrund ab. Die Dosen der beruflich strahlenexponierten Personen sind zumeist in der gleichen Größenordnung. Im Jahre 1998 überschritten nur ca. 1 \% der gemessenen Ganzkörperdosen über- 
wachter Personen einen Wert von $5 m S v$ [12]. Aus diesen Werten lässt sich berechnen, dass der überwiegende Anteil der Körperzellen, die im Laufe ihrer Existenz einer Dosis durch dichtionisierende Strahlung ausgesetzt sind, den Durchgang von genau einem dichtionisierenden Teilchen erfahren. Die Untersuchung der Strahlenwirkung einzelner Teilchen ist daher von großer Bedeutung für die Beurteilung natürlicher, zivilisatorischer und beruflicher Strahlenexpositionen.

\subsection{Motivation und Ziel}

Tumoren entstehen aus einer gesunden Zelle durch eine Abfolge von mehreren Schritten, die nach derzeitigem Kenntnisstand Mutationen in Tumorgenen sind, wodurch genomische Instabilität induziert wird. Dies hat Chromosomenaberrationen oder sogar Chromosomenverluste zur Folge. Wenn durch eine spezifische Abfolge von Mutationen eine bösartige Zelle entstanden ist, so sind eine Reihe von weiteren Schritten bei deren Wachstum und Ausbreitung im Gewebe und im Gesamtorganismus notwendig, um Krebs hervorzubringen. Der Mehrschrittmechanismus der Karzinogenese hat zur Folge, dass die karzinogene Wirkung, beispielsweise einer Strahlenexposition, mit einer Verzögerung von oftmals vielen Jahren auftritt, wie es bei den Überlebenden von Hiroshima und Nagasaki beobachtet wurde [7]. Weiterhin muss berücksichtigt werden, dass der Hintergrund von bereits vorhandenen Mutationen im Organismus von Individuum zu Individuum verschieden ist und dass im jeweiligen Individuum von einer Verteilung der Körperzellen im Hinblick auf Mutationen in Tumorgenen ausgegangen werden muss. Dieser Sachverhalt erschwert die Abschätzung des Krebsrisikos durch ionisierende Strahlung auf der Basis epidemiologischer Studien. Es ist deshalb notwendig, die Abschätzung des Strahlenrisikos mithilfe von Erkenntnissen über die molekularen und zellularen Mechanismen, die zur Strahlenkarzinogenese führen, zu präzisieren.

Daher sind in vitro Bestrahlungen von Zellen oder Geweben seit vielen Jahren ein wichtiges Mittel in der radiobiologischen Forschung. Hierbei werden Zellen einem Strahlungsfeld, dessen Eigenschaften wie z.B. LET und Dosisleistung gut bekannt und messbar sind, ausgesetzt. Bei herkömmlicher Bestrahlung von Zellen mit dicht ionisierenden Teilchen werden die Zellen poissonverteilt getroffen, so dass in Bezug auf biologische Strahlenwirkung nur eine stochastische Aussage getroffen werden kann. Die relative Abweichung der tatsächlichen Dosis einer Zelle von der 
mittleren Dosis ist hierbei umso größer, je niedriger die Dosis ist. Zudem ist eine statistisch signifikante Auswirkung der Strahlung oftmals erst bei hohen Dosen messbar.

Eine wesentliche Verbesserung der experimentellen Bedingungen ergibt sich, wenn man die Dosis jeder einzelnen Zelle messen oder einstellen kann, und zudem die räumliche Dosisverteilung genauer kennt. Wenn man auf diese Weise die Stochastik ausschaltet, wird eine Präzisierung der Abfolge der angeführten biologischen Mechanismen und eine bessere Untersuchung der kausalen Zusammenhänge zwischen primären physikalischen Effekten und der biologischen Wirkung möglich. Ziel dieser Arbeit ist es, eine solche Bestrahlungsapparatur aufzubauen und erste Messungen durchzuführen. Zu Beginn der Arbeit wurden die Zielvorgaben aus Tabelle 1.2 für die Anlage festgelegt.

\begin{tabular}{|l|l|}
\hline Ortsauflösung & $\leq 1 \mu \mathrm{m}$ \\
Nachweiswahrscheinlichkeit der Ionen & $>95 \%$ \\
Teilchenrate im Experiment & $<1000 \mathrm{~s}^{-1}$ \\
Maximaler Strahlstrom & $>1 \mathrm{pA}$ \\
Schaltzeit des beam shutters & $<50 \mu \mathrm{s}$ \\
Durchsatz von bestrahlten Zellen & $\geq 500 \mathrm{~h}^{-1}$ \\
\hline
\end{tabular}

Tabelle 1.2: Angestrebte Parameter des Mikro-Ionenstrahls.

Einzelteilchen-Experimente mit hoher Ortsauflösung erlauben es, die Wirkung der ultimativ geringsten „Dosis" ${ }^{\star 6}$, nämlich der, hervorgerufen durch ein einzelnes Ion, zu untersuchen. Dies wird durch zwei wesentliche Unterschiede zu herkömmlicher Bestrahlung erreicht. Erstens kann die Strahlenbelastung jeder einzelnen Zelle genau auf ein Teilchen eingestellt werden. Zweitens wird der Stichprobenumfang für ein wohldefiniertes primäres Ereignis vergrößert, selbst wenn durch die experimentellen Gegebenheiten im Mikrostrahlexperiment nur eine geringe Anzahl von Zellen bestrahlt wird. So konnte z.B. durch die gezielte Bestrahlung der sensiblen Strukturen (DNA) selbst bei dem Durchgang eines einzelnen $\alpha$-Teilchens eine signifikante Zellantwort experimentell nachgewiesen werden [13].

Durch die hohe Ortsaufösung kann die Strahlensensitivität verschiedener Strukturen in einer Zelle untersucht werden. Mikrostrahl-Untersuchungen können so

\footnotetext{
${ }^{6}$ Der Dosisbegriff ist bei solchen Experimenten nicht adäquat, da er eine über ein makroskopisches Volumen gemittelte Größe darstellt.
} 
eine experimentelle Datengrundlage für verfeinerte mechanistische Modellrechnungen in der Mikro- und Nanodosimetrie liefern. Zur Zeit wird bei diesen noch vorausgesetzt, dass nur die Schädigung der Kern-DNA eine biologische Wirkung hervorruft. Neue Mikrostrahl-Untersuchungen zeigen eine Wirkung auch bei Bestrahlung des Zellplasmas oder gar des Nährmediums [14].

In den letzten Jahren wurden in der strahlenbiologischen Forschung im niedrigen Dosisbereich mehrere Phänomene beobachtet, die sowohl für den Strahlenschutz als auch für die Strahlentherapie von Bedeutung sein können: Die adaptive Zellantwort (engl.: adaptive response) und der 'bystander effect'.

Zellen zeigen eine adaptive Zellantwort, wenn sie nach einer kleinen Energiedosis (im Allgemeinen geringer als 0,2 Gy) und geeignetem zeitlichen Abstand zur eigentlichen Bestrahlungsdosis eine geringere strahlenbiologische Wirkung zeigen als ohne diese Vorbestrahlung [15]. Mit 'bystander effect' wird die Beobachtung bezeichnet, dass von Teilchen nicht getroffene Nachbarzellen eine Strahlenreaktion zeigen. Dieser Effekt wurde bisher für die Zellinaktivierung, die Mutation und die neoplastische Zelltransformation beschrieben [16, 17, 18]. Die molekularbiologischen Mechanismen dieser strahlenbiologischen Phänomene sind praktisch nicht bekannt. Der Mikro-Ionenstrahl stellt auch für diese Phänomene, die ja gerade im niedrigen Dosisbereich beobachtet werden, ein wichtiges Forschungsinstrument dar, um wesentliche Beiträge für ihre Aufklärung zu leisten.

Im Gegensatz zu herkömmlichen Bestrahlungstechniken erlauben Mikrostrahlexperimente auch den Einfluss der Zeitstruktur der Ionisationsereignisse zu untersuchen, da es möglich ist, die zeitliche Abfolge der Teilchen, die eine bestimmtes Zellkompartiment treffen, festzulegen und zu variieren. Diese Eigenschaft ist für die Untersuchung der enzymatischen Reparatur- und Falschreparaturprozesse, die in der fraktionierten Strahlentherapie eine wichtige Rolle spielen, von außerordentlicher Bedeutung.

Die Aufzählung und Erläuterung der Beispiele zeigen, dass der Mikro-Ionenstrahl für die radiobiologische Forschung ein wichtiges Instrument sein wird. Durch Mikrostrahl-Experimente lassen sich die Zusammenhänge zwischen den physikalischen Primäreffekten der verschiedenen Strahlenarten und den ausgelösten biologischen Reaktionen genauer als bisher untersuchen. Ziel ist es, die Strahlenwirkung bis hin zur Strahlenkarzinogenese bzw. zur Inaktivierung von Zellen (Hemmung der Zellproliferation) im Bereich kleiner Dosen verstehen zu lernen. 


\subsection{Die Ionenbeschleunigeranlage der PTB}

Die Fachabteilung 6 der Physikalisch-Technischen Bundesanstalt befasst sich mit der Metrologie ionisierender Strahlung. Diese hat große Bedeutung in der medizinischen Diagnostik und Therapie, im Bereich der beruflich strahlenexponierten Personen und in der Umweltüberwachung. Ein wichtiger Teilbereich ist die Darstellung der Dosisgrößen für den Strahlenschutz sowie die Zulassung und Kalibrierung von Strahlenschutzdosimetern. Als Quelle für ionisierende Strahlung stehen in der PTB verschiedene Beschleuniger sowie unterschiedliche Radionuklidquellen zur Verfügung.

Das Labor Neutronenmetrologie betreibt zwei Teilchenbeschleuniger, einen Vande-Graaff Generator (VdG) und ein Isochron-Zyklotron, die hochenergetische Protonen, Deuteronen und ${ }^{4} H e^{2+}-$ Kerne ${ }^{7}$ liefern können (s. Tabelle 1.3). Der Strahlstrom beträgt für Protonen und Deuteronen maximal $50 \mu \mathrm{A}$ bis $100 \mu \mathrm{A}$ bei Emittanzen zwischen $3 \mathrm{~mm}$ mrad und $8 \mathrm{~mm}$ mrad in den beiden transversalen Koordinaten. Die Beschleuniger werden in der Hauptsache dazu verwendet, verschiedene Neutronenfelder darzustellen [20].

\begin{tabular}{|c|c|c|c|c|c|c|}
\hline Teilchen & Energie / MeV & \multicolumn{2}{|c|}{$\mathrm{LET} / \mathrm{keV} / \mu \mathrm{m}$} & \multicolumn{3}{|c|}{ Reichweite / $\mu m$} \\
\hline Protonen & $0,2 \quad-$ & $3-$ & 70 & 3 & - & 4200 \\
\hline Deuteronen & 0,3 & 7 & 80 & 6 & - & 1100 \\
\hline${ }^{4} H e^{2+}$-Kerne & 0,4 & 25 & 200 & 3 & - & 630 \\
\hline
\end{tabular}

Tabelle 1.3: Teilchensorten und Energien am PTB Ionenbeschleuniger. Die Werte für LET und Reichweite beziehen sich auf Wasser.

Die Ionenbeschleunigeranlage der PTB ist in Abbildung 1.2 schematisch dargestellt. Der VdG-Generator befindet sich im Erdgeschoss. Mittels zweier Quadrupoldubletts und einem $90^{\circ}$-Dipol-Magneten wird der VdG-Strahl zum Schaltmagneten geführt, der den Strahl in die verschiedenen Strahlrohre lenken kann. Der 90-Dipol-Magnet dient zusammen mit zwei Schlitzpaaren als Analysiersystem. Hiermit kann die Strahlenergie bestimmt und die Terminalspannung des VdG nachgeregelt werden. Das energievariable Zyklotron befindet sich im Kellergeschoss. Mittels zweier $90^{\circ}$-Dipol-Magneten und mehrerer magnetischer Linsen wird der Zyklotronstrahl zum Schaltmagneten geführt und kann dort ebenfalls in

\footnotetext{
${ }^{7}{ }^{4} \mathrm{He}^{2+}$-Kerne werden im Folgenden auch als $\alpha$-Teilchen bezeichnet, da sie mit diesen von einigen Radioisotopen emittierten Teilchen physikalisch identisch sind.
} 


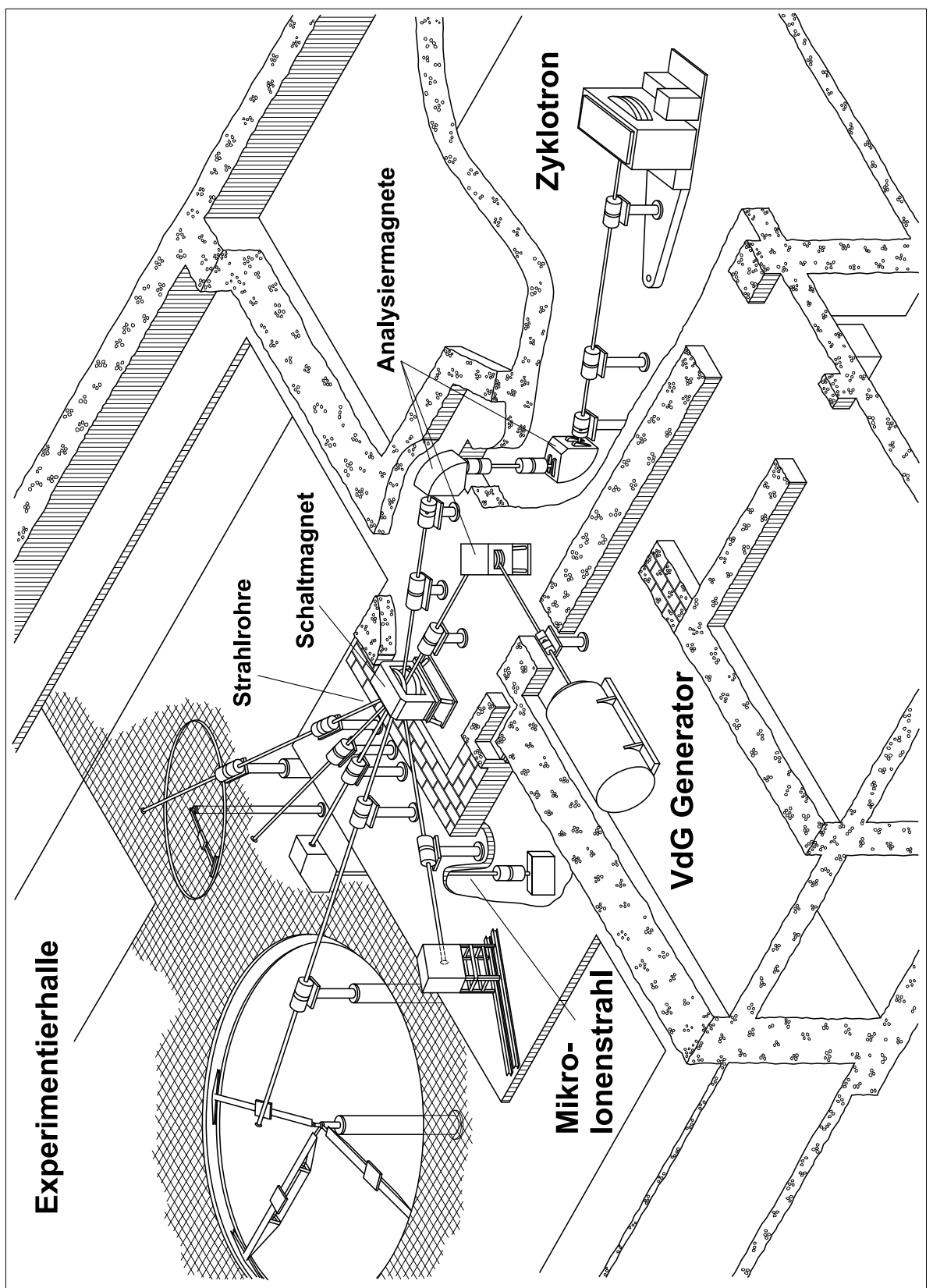

Abbildung 1.2: Übersicht über die Ionenbeschleunigeranlage der PTB.

die verschiedenen Strahlrohre gelenkt werden. In $0^{\circ}$-Richtung zum VdG-Strahl befindet sich das neue Strahlrohr 4, das den Mikro-Ionenstrahl beherbergt (s. Abb. 1.2). In der Experimentierhalle wurde ein 6,80 $\mathrm{m}$ hohes, massives Stahlgerüst aufgebaut, das einen Dipolmagneten trägt, der den Ionenstrahl um $90^{\circ}$ nach unten ablenkt. Der Experimentierplatz des Ionenstrahls befindet sich im Kellergeschoss der Experimentierhalle, etwa $1 \mathrm{~m}$ über dem Hallenboden. 
Eine wichtige Eigenschaft ist der weite LET-Bereich von $3 \mathrm{keV} / \mu \mathrm{m}$ bis $200 \mathrm{keV} / \mu \mathrm{m}$, der am Ionenbeschleunigerlabor der PTB zur Verfügung steht. Die für den Strahlenschutz relevanten Strahlenarten haben zumeist ein LET zwischen $1 \mathrm{keV} / \mu \mathrm{m}$ (Röntgenstrahlen) und $100 \mathrm{keV} / \mu \mathrm{m}$ ( $\alpha$-Teilchen). Höhere Ionisationsdichten kommen nur am Ende der Reichweite eines Teilchens (Bragg-Peak) und bei schweren Teilchen vor, welche auf der Erde nur künstlich erzeugt werden können. Mit dem PTB Mikro-Ionenstrahl ist es also möglich, biologische Experimente in einem weiten und zudem strahlenschutzrelevanten LET-Bereich durchzuführen. Hochenergetische Protonen können hierbei die Wirkung von $\gamma$-Strahlen simulieren, da sie ein ähnliches LET besitzen.

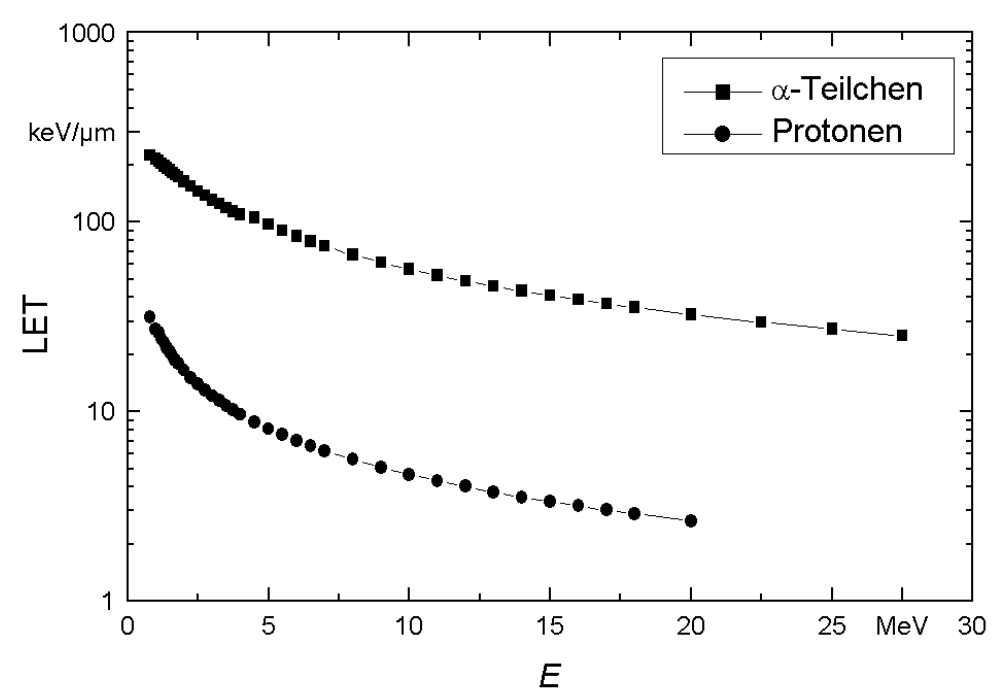

Abbildung 1.3: Das LET der Ionen der PTB in Wasser.

\subsection{Prinzipieller Aufbau des Mikro-Ionenstrahls}

Die Idee, Zellen mit einzelnen Ionen gezielt zu bestrahlen, reicht viele Jahre zurück [21]. Aber erst in den 90er Jahren ist es einigen Forschungseinrichtungen gelungen, Mikrostrahlexperimente im Routinebetrieb durchzuführen [22, 23, 24]. Weltweit gibt es nur eine kleine Zahl von Einrichtungen, die radiobiologische Mikrostrahlexperimente durchführen können. Die Tabelle 1.4 gibt einen Überblick über die weltweit betriebsbereiten Anlagen. 


\begin{tabular}{|l|l|r|c|l|}
\hline Institut & Strahlenart & $\begin{array}{r}\text { Energie } \\
\text { LET }\end{array}$ & Status \\
\hline $\begin{array}{l}\text { Gray Laboratory } \\
\text { (London) }\end{array}$ & $\begin{array}{l}\text { Protonen } \\
{ }^{3} \mathrm{He}^{2+},{ }^{4} \mathrm{He}^{2+}\end{array}$ & $\begin{array}{r}4 \mathrm{MeV} \\
(\mathrm{VdG})\end{array}$ & hoch & $\begin{array}{l}\text { Betrieb seit } \\
\text { ca. } 1996\end{array}$ \\
\hline Gray Laboratory & weiche & $270 \mathrm{eV}$ & mittel & Betrieb seit \\
(London) & Photonen & $-1,5 \mathrm{keV}$ & & ca. 2000 \\
\hline Columbia University & Protonen & $4 \mathrm{MeV}$ & hoch & Betrieb seit \\
(New York) & ${ }^{3} \mathrm{He}^{2+},{ }^{4} \mathrm{He}^{2+}$ & $(\mathrm{VdG})$ & & ca. 1994 \\
\hline JAERI (Tokyo) & schwere & 17,5 & hoch & Betrieb seit \\
& Ionen & $\mathrm{MeV} / u$ & & ca. 1998 \\
\hline PNNL (Seattle) & Elektronen & $80 \mathrm{kV}$ & niedrig & fertiggestellt \\
\hline
\end{tabular}

Tabelle 1.4: Mikrostrahlen in der radiobiologischen Forschung. Neben den aufgezählten befinden sich weitere Anlagen in Planung oder im Aufbau [26].

Um einen Ionenstrahl räumlich einzuengen, gibt es im Wesentlichen zwei Möglichkeiten: die Kollimation des Strahls durch mechanische Elemente und die Fokussierung des Strahls mittels elektrischer oder magnetischer Linsen. Die Kollimation ist für Ionen mit hohem LET die adäquate Methode. Neben der Begrenzung des Strahldurchmessers auf die Ausmaße des Kollimators beinhaltet sie die Festlegung der Strahlposition am Kollimatorausgang und die Reduzierung des Strahlstroms auf für radiobiologische Experimente sinnvolle Werte. Mit einem mikrokollimierten Ionenstrahl werden 1 bis $5 \mu \mathrm{m}$ Ortsauflösung für dichtionisierende Teilchen erreicht [25]. Ein Nachteil der Kollimation ist der unvermeidliche Anteil von gestreuten Teilchen, der hierbei entsteht und welcher die Orts- und Energieauflösung beeinträchtigt. Zudem ist eine Variation des Strahlstroms, insbesondere eine Erhöhung nur eingeschränkt möglich.

Für weniger dicht ionisierende Ionen, z.B. für Protonen mit mehr als $5 \mathrm{MeV}$, ist die Kollimation keine geeignete Methode, da diese Teilchen erhebliche Eindringtiefen in Material besitzen, was eine effektive Kollimation verhindert (s. Reichweiten in Tabelle 1.3). Hier können die fokussierenden Eigenschaften von Magneten genutzt werden, um einen Ionenstrahl räumlich einzuengen. Bei geeigneter Wahl der Magnete lassen sich die Ionen der verschiedenen Sorten und Energien der PTB Ionenbeschleuniger mit ein und demselben Strahlführungsystem fokussieren. Die Stärken der Magnetfelder müssen dazu mit der magnetischen Steifigkeit der betreffenden Ionen skaliert werden.

Um die volle Bandbreite der zur Verfügung stehenden Ionen und deren Energien für den Mikrostrahl auszunutzen, wurde das Prinzip der Fokussierung gewählt. 
Neben den oben beschriebenen Argumenten spielt hierbei auch die größere Flexibilität eines fokussierten Strahls für mögliche weitere Anwendungen eine Rolle. Die Anforderungen der Experimente und die baulichen Gegebenheiten in der Experimentierhalle lassen für die Lage des Bestrahlungsplatzes nur den Hallenboden aus Beton zu (s. Abb. 1.4). Das Erdgeschoss der Experimentierhalle besteht im wesentlichen aus Drahtgitter, eine Forderung für Neutronenexperimente, bei denen eine streuarme Umgebung benötigt wird. Ein vibrationsarmer Aufbau ist deshalb im Erdgeschoss nicht möglich. Die Details werden in den folgenden Kapiteln ausführlich erläutert. An dieser Stelle sollen nur einige wesentliche Merkmale des Aufbaus skizziert werden.

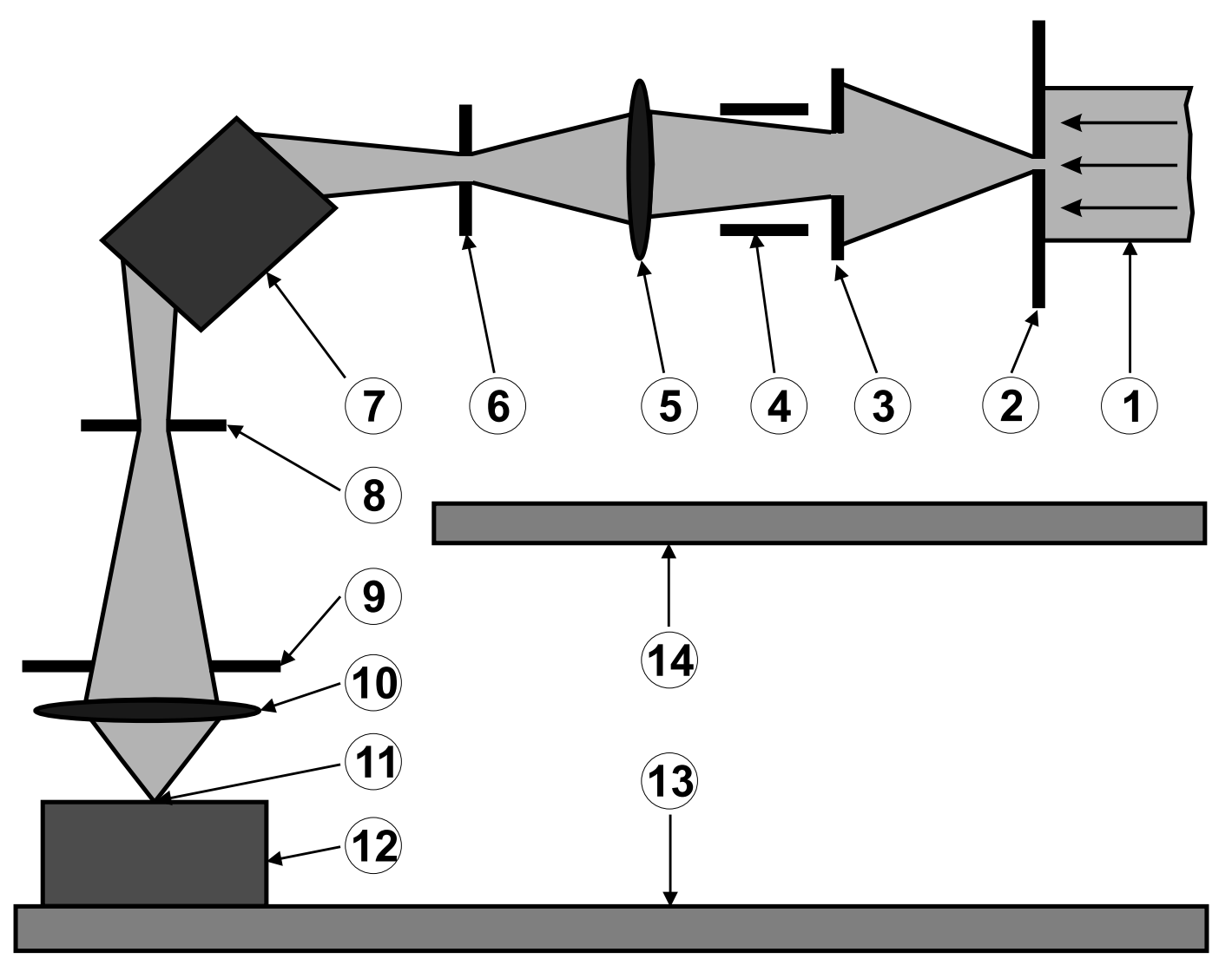

Abbildung 1.4: Prinzipieller Aufbau des Mikro-Ionenstrahls (Strahlrohr 4) mit den wesentlichen Strahlführungselementen.

- Der einkommende Ionenstrahl (1) wird durch die Objektblende (2) auf wenige Mikrometer eingeschränkt. Die folgenden ionenoptischen Elemente bilden die Objektblende verkleinert auf den Bildpunkt am Ende der Strahlführung ab. 
- Der Divergenzschlitz (Schlitz 41) (3) engt den Winkelbereich des Ionenstrahls ein und ermöglicht die Reduktion des Strahlstroms.

- Der elektrostatische Deflektor (4) erlaubt es, den Strahl schnell ein- und auszuschalten (beam shutter).

- Das Quadrupoldublett (5) im horizontalen Teil der Strahlführung fokussiert den Strahl.

- Der Dipolmagnet (7) lenkt den Strahl mit einem Biegeradius von $75 \mathrm{~cm}$ um $90^{\circ}$ senkrecht nach unten.

- Die Energieschlitze (Schlitz 42 \& 43) (6, 8) ermöglichen im Zusammenspiel mit dem Dipolmagneten die energieselektive Abbildung des Ionenstrahls.

- Die Anti-Streu-Schlitze (Schlitz 44) (9) eliminieren Streuteilchen, die sich weit entfernt von der Sollbahn befinden, ohne den Sollstrahl zu beeinträchtigen.

- Alle Schlitze $(3,6,8,9)$ dienen darüber hinaus auch als Messinstrumente für die Strahllage, da der Strom auf jeder Schlitzbacke gemessen wird.

- Ein Quadrupoldublett (10) mit hohem Feldgradienten fokussiert den Ionenstrahl mit einer Bildweite von weniger als $20 \mathrm{~cm}$.

- Der Ionenstrahl tritt durch ein Vakuumfenster (11) aus dem Vakuum aus.

- Der Experimentierplatz (12) mit der zu bestrahlenden biologischen Probe befindet sich im Untergeschoss der Experimentierhalle (13).

$\mathrm{Zu}$ Beginn der Arbeit stand ein ungenutzter Erweiterungsflansch hinter dem Schaltmagneten zur Verfügung. An diesen anschließend wurde die Strahlführung des Mikro-Ionenstrahls konzipiert und aufgebaut. Als vorbereitende Arbeiten waren bereits die Bestellung des Dipolmagneten und des ersten Quadrupoldubletts sowie die Konstruktion des Stahlgerüstes für den Dipolmagneten erledigt.

Die Aufgabenstellung dieser Arbeit umfasst daher:

- Konzeption der Strahlführung

- Berechnung der optischen Eigenschaften 
- Spezifizierung und Beschaffung oder Konstruktion der Komponenten

- Entwicklung geeigneter Strahldiagnoseinstrumente

- Aufbau des Experimentierplatzes

- Test der Anlage und Optimierung der Parameter

- Vorbereitung und Durchführung erster biologischer Experimente in Kooperation mit den Nutzern aus der Radiobiologie 


\section{Kapitel 2}

\section{Grundlagen der Ionenoptik}

In Anlehnung an die Terminologie der Geometrischen Optik bezeichnet man die Strahlführung von Teilchenstrahlen als optische Abbildung. In diesem Kapitel werden die grundlegenden Begriffe und Verfahren erläutert, die für das weitere Verständnis notwendig sind. Umfassendere Beschreibungen sind in den Arbeiten von Brown und Hinterberger zu finden [27, 28].

\subsection{Koordinatensystem}

Die Bahn, die ein Teilchen im idealen Fall im Beschleuniger beziehungsweise im Strahlrohr durchläuft, wird Sollbahn oder Orbit genannt. Die Koordinaten eines Teilchens werden relativ zu dieser Sollposition angegeben. Im Allgemeinen wird ein Teilchen an der Stelle $s$ eine Ortsabweichung $x(s), y(s)$ und einen Bahnwinkel $x^{\prime}(s), y^{\prime}(s)$ bezüglich der Sollbahn haben. Neben diesen transversalen Komponenten muss man im Falle eines zeitlich gepulsten Strahls noch die longitudinale Abweichung zur Sollposition $l(s)$ betrachten. Als weitere Größe ist der Impuls eines Teilchens wichtig. Auch hier wird der Impuls auf den Sollimpuls $p_{0}$ bezogen, so dass als charakteristische Größe die relative Impulsabweichung $\delta=\left(p-p_{0}\right) / p_{0}$ verwendet wird. Ein Teilchen wird demnach durch einen Zustandsvektor mit folgenden Größen beschrieben:

$$
r(s)=\left(\begin{array}{c}
r_{1} \\
r_{2} \\
r_{3} \\
r_{4} \\
r_{5} \\
r_{6}
\end{array}\right)=\left(\begin{array}{c}
x \\
x^{\prime} \\
y \\
y^{\prime} \\
l \\
\delta
\end{array}\right)=\left(\begin{array}{c}
\text { horizontale Ortsabweichung }(\mathrm{mm}) \\
\text { horizontale Richtungsabweichung }(\mathrm{mrad}) \\
\text { vertikale Ortsabweichung }(\mathrm{mm}) \\
\text { vertikale Richtungsabweichung }(\mathrm{mrad}) \\
\text { longitudinale Ortsabweichung }(\mathrm{mm}) \\
\text { relative Impulsabweichung }(\% \mathrm{o0})
\end{array}\right)
$$




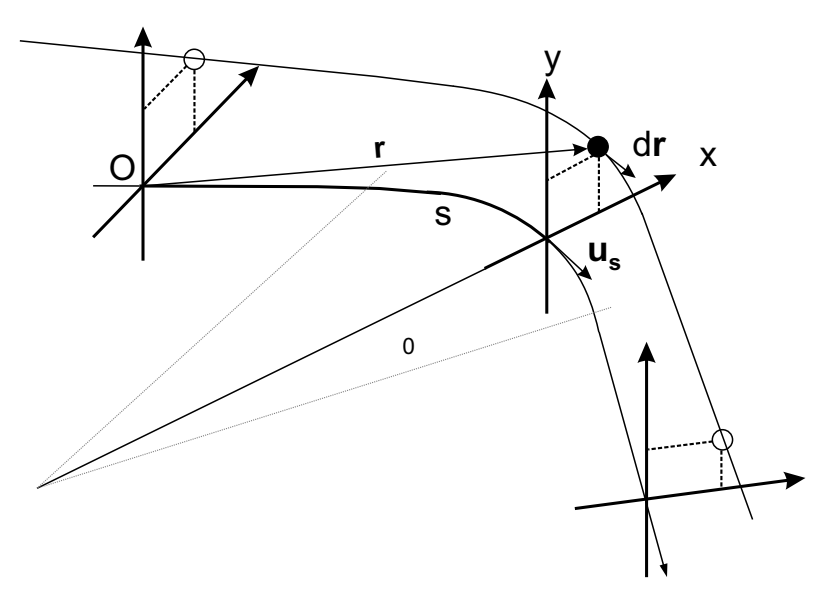

Abbildung 2.1: Koordinatensystem [28].

Das Linienelement im mitbewegten Koordinatensystem (s. Abbildung 2.1) ist gegeben durch

$$
d \vec{r}=\hat{\vec{x}} d x+\hat{\vec{y}} d y+\hat{\vec{s}}(1-h x) d s
$$

wenn $\hat{\vec{x}}, \hat{\vec{y}}, \hat{\vec{s}}$ die Orthonormalvektoren im mitbewegten Koordinatensystem und $h$ die Krümmung der Sollbahn ( $h=1 / \rho_{0}, \rho_{0}=$ Krümmungsradius) bedeuten. Es werden nur Sollbahnen betrachtet, die eine Krümmung in der y-Richtung aufweisen. Diese Richtung wird auch radial genannt, die Richtung ohne Sollbahnkrümmung axial.

\subsection{Bahngleichungen}

Verwendet man in einem Strahlführungssystem nur magnetische Elemente, so wird die Bewegung geladener Teilchen durch die Lorentzkraft bestimmt:

$$
\dot{\vec{p}}=q \vec{v} \times \vec{B}
$$

Bei der Beschränkung auf statische Magnetfelder ist der Betrag der Geschwindigkeit der Teilchen konstant. Somit kann man die zeitliche Ableitung durch die Ableitung nach dem Ortsvektor entlang der Teilchenbahn ersetzen:

$$
\frac{d}{d t}=v \frac{d}{d r}
$$


Transformiert man die Gleichung für die Lorentzkraft 2.3 mit der Beziehung 2.4, erhält man folgende Differentialgleichung, in der keine zeitliche Ableitung vorkommt:

$$
\frac{d^{2} \vec{r}}{d r^{2}}=\frac{q}{p} \frac{d \vec{r}}{d r} \times \vec{B}
$$

In dieser Gleichung muss das Bogenelement der zu beschreibenden Bahn $d r$ durch das Bogenelement der Sollbahn $d s$ ersetzt werden:

$$
\vec{r}^{\prime \prime}-\frac{1}{2} \frac{\vec{r}^{\prime}}{r^{\prime 2}} \frac{d}{d s}\left(r^{\prime}\right)^{2}=\frac{q}{p} r^{\prime}\left(\vec{r}^{\prime} \times \vec{B}\right) .
$$

Wird diese Gleichung in den Koordinaten des oben beschriebenen mitbewegten Koordinatensystems dargestellt, so ergibt sich nach einigen Rechenschritten [27]:

$$
\begin{aligned}
& \hat{\vec{x}}\left\{\left[x^{\prime \prime}-h(1+h x)\right]-\frac{x^{\prime}}{\left(r^{\prime}\right)^{2}}\left[x^{\prime} x^{\prime \prime}+y^{\prime} y^{\prime \prime}+(1+h x)\left(h x^{\prime}+h^{\prime} x\right)\right]\right\} \\
+ & \hat{\vec{y}}\left\{y^{\prime \prime}-\frac{y^{\prime}}{\left(r^{\prime}\right)^{2}}\left[x^{\prime} x^{\prime \prime}+y^{\prime} y^{\prime \prime}+(1+h x)\left(h x^{\prime}+h^{\prime} x\right)\right]\right\} \\
+ & \hat{\vec{s}}\left\{\left(2 h x^{\prime}-h^{\prime} x\right)-\frac{1+h x}{\left(r^{\prime}\right)^{2}}\left[x^{\prime} x^{\prime \prime}+y^{\prime} y^{\prime \prime}+(1+h x)\left(h x^{\prime}+h^{\prime} x\right)\right]\right\} \\
= & \frac{q}{p} r^{\prime}\left(\vec{r}^{\prime} \times \vec{B}\right) \\
= & \frac{q}{p} r^{\prime}\left\{\hat{\vec{x}}\left[y^{\prime} B_{s}-(1+h x) B_{y}\right]+\hat{\vec{y}}\left[(1+h x) B_{x}-x^{\prime} B_{s}\right]+\hat{\vec{s}}\left[x^{\prime} B_{y}-y^{\prime} B_{x}\right]\right\} .
\end{aligned}
$$

Diese Gleichung gilt exakt. In der Praxis reicht es meist aus, nur die Terme, die linear oder quadratisch in den Ortskoordinaten und deren Ableitungen sind (1. und 2. Ordnung), zu berücksichtigen.

Setzt man in diese Gleichung die Koordinaten der Sollbahn $\left(x=x^{\prime}=x^{\prime \prime}=y=\right.$ $\left.y^{\prime}=y^{\prime \prime}=0 ; p=p_{0}\right)$ ein, so ergibt sich:

$$
h(s)=\frac{q}{p_{0}} B_{y}(0,0, s) \quad \text { und } \quad 0=B_{x}(0,0, s)
$$

was die oben angenommene Bedingung der Krümmung nur in y-Richtung beschreibt. Die longitudinale Magnetfeldkomponente $B_{s}$ bleibt hierbei unbestimmt, da sie auf ein Sollteilchen keinen Einfluss hat. 


\subsection{Magnetfelder in der Strahloptik}

In der Strahloptik sind die folgenden drei ionenoptischen Elemente von großer Bedeutung:

- Driftstrecke: magnetfeldfreier Raum, $\vec{B}=\overrightarrow{0}$.

- Homogener Ablenkmagnet: räumlich konstantes Magnetfeld, $\vec{B}=\left(0, B_{y}, 0\right)=\left(0, \frac{p_{0}}{q \rho_{0}}, 0\right)$.

- Quadrupolmagnet: fokussierendes und defokussierendes Magnetfeld, $B_{x}=g y, B_{y}=g x, B_{z}=0$.

Vernachlässigt man alle Terme ab der 2. Ordnung und benutzt die Entwicklung $1 / p=(1-\delta) / p_{0}$ und $r^{\prime}=1+h x$, so ergibt sich aus den Gleichungen 2.7 für die Driftstrecke:

$$
\begin{aligned}
& x^{\prime \prime}=0, \\
& y^{\prime \prime}=0 .
\end{aligned}
$$

Im feldfreien Raum sind die Bahnkurven Geraden.

Für den homogenen Ablenkmagneten folgt:

$$
\begin{aligned}
x^{\prime \prime}+h^{2} x & =h \delta, \\
y^{\prime \prime} & =0
\end{aligned}
$$

und für den Quadrupolmagneten (Singulett)

$$
\begin{aligned}
& x^{\prime \prime}+\frac{q g}{p_{0}} x=0, \\
& y^{\prime \prime}-\frac{q g}{p_{0}} y=0 .
\end{aligned}
$$

Man beachte, dass die zweiten Terme in den Gleichungen 2.11 unterschiedliche Vorzeichen haben. Das entspricht der Tatsache, dass ein Quadrupol in einer Richtung fokussierend und in der anderen defokussierend wirkt. Durch das Vorzeichen von $g$ wird festgelegt, in welcher Richtung fokussiert wird. 


\subsection{Allgemeine Lösung in 1. Ordnung}

Die transversale Bahngleichung hat in 1. Ordnung die allgemeine Form [28]:

$$
\begin{aligned}
x^{\prime \prime}+k_{x} x & =h \delta, \\
y^{\prime \prime}+k_{y} y & =0 .
\end{aligned}
$$

Die Koeffizienten $k_{x}, k_{y}$ und $h$ werden durch Form und Stärke des jeweiligen Magnetfeldes bestimmt.

Man wählt für jede Koordinate zwei linear unabhängige Lösungen der homogenen Gleichungen (2.12), die folgende Anfangsbedingungen erfüllen:

$$
\begin{array}{ll}
c_{x}(0)=1 & c_{x}^{\prime}(0)=0 \\
s_{x}(0)=0 & s_{x}^{\prime}(0)=1 \\
c_{y}(0)=1 & c_{y}^{\prime}(0)=0 \\
s_{y}(0)=0 & s_{y}^{\prime}(0)=1 .
\end{array}
$$

Die Funktionen $s_{i}$ werden sinusartig, die $c_{i}$ kosinusartig genannt.

Beliebige Lösungen der homogenen Gleichung lassen sich dann als Linearkombination schreiben (für x entsprechend).

$$
\left(\begin{array}{c}
y(s) \\
y^{\prime}(s)
\end{array}\right)=\left(\begin{array}{ll}
c_{y} & s_{y} \\
c_{y}^{\prime} & s_{y}^{\prime}
\end{array}\right)\left(\begin{array}{c}
y(0) \\
y^{\prime}(0)
\end{array}\right) .
$$

Wenn die Impulsabweichung $\delta$ eines Teilchens nicht verschwindet, so ergibt sich für den Ablenkmagneten (Sollbahnkrümmung $h \neq 0$ ) eine inhomogene Gleichung. $\mathrm{Zu}$ deren Lösung muss eine partikuläre Lösung der inhomogenen Gleichung $\delta d_{x}(s)$ gefunden werden. Dies geschieht mittels der Greenschen Funktion [28]

$$
\begin{aligned}
d_{x}(s) & =\int_{0}^{s} h(\bar{s}) G_{x}(s, \bar{s}) d \bar{s} \\
& =s_{x}(s) \int_{0}^{s} h(\bar{s}) c_{x}(\bar{s}) d \bar{s}-c_{x}(s) \int_{0}^{s} h(\bar{s}) s_{x}(\bar{s}) d \bar{s} .
\end{aligned}
$$

Die Funktion $d_{x}(s)$ wird Dispersion genannt und erfüllt die Anfangsbedingungen

$$
d_{x}(0)=0, d_{x}^{\prime}(0)=0
$$

Eine allgemeine Lösung in $\mathrm{x}$-Richtung lässt sich somit wie folgt schreiben:

$$
\left(\begin{array}{c}
x(s) \\
x^{\prime}(s) \\
\delta
\end{array}\right)=\left(\begin{array}{ccc}
c_{x} & s_{x} & d_{x} \\
c_{x}^{\prime} & s_{x}^{\prime} & d_{x}^{\prime} \\
0 & 0 & 1
\end{array}\right)\left(\begin{array}{c}
x(0) \\
x^{\prime}(0) \\
\delta
\end{array}\right) .
$$




\subsection{Matrixschreibweise}

Wie in Abschnitt 2.4 gezeigt wurde, kann man die Lösung der Differentialgleichung in linearer Näherung durch Matrixmultiplikation der charakteristischen Lösungen mit dem Anfangsvektor der Phasenraumkoordinaten erhalten. Somit kann man die Transformation der Teilchenkoordinaten durch ein ionenoptisches Element als $6 \times 6$ Matrix schreiben. 1

$$
x(s)=R(s) x(0) .
$$

Man kann ein Strahlführungssystem in Abschnitte mit konstantem Magnetfeldverlauf unterteilen und die Bahngleichungen für jeden dieser Abschnitte lösen. Weil der Ausgangsvektor eines Abschnittes als Anfangsvektor für den nächsten dient, kann man die Lösung eines Systems mit mehreren aufeinanderfolgenden Abschnitten einfach durch sukzessive Matrixmultiplikation errechnen. Da die Matrixmultiplikation assoziativ ist, ist die Transformationsmatrix eines optischen Systems von zwei aufeinander folgenden Elementen durch das Produkt der Transformationsmatrizen gegeben:

$$
R\left(s_{0} \rightarrow s_{2}\right)=R\left(s_{1} \rightarrow s_{2}\right) R\left(s_{0} \rightarrow s_{1}\right)
$$

Im Falle der Ausrichtung aller magnetischen Komponenten an den gleichen Achsen haben Transformationsmatrizen zumeist das Aussehen wie in Gleichung 2.20 [28]. Einige Matrixelemente betragen in jedem Fall 0, da z.B. die beiden transversalen Richtungen voneinander unabhängig sind. Die Matrixelemente $R_{56}$ und $R_{66}$ betragen 1, da Magnetfelder keine Änderung des Betrages des Impulses hervorrufen können.

$$
R=\left(\begin{array}{cccccc}
R_{11} & R_{12} & 0 & 0 & 0 & R_{16} \\
R_{21} & R_{22} & 0 & 0 & 0 & R_{26} \\
0 & 0 & R_{33} & R_{34} & 0 & 0 \\
0 & 0 & R_{43} & R_{44} & 0 & 0 \\
R_{51} & R_{52} & 0 & 0 & 1 & R_{56} \\
0 & 0 & 0 & 0 & 0 & 1
\end{array}\right)
$$

\footnotetext{
${ }^{1}$ Die longitudinale Koordinate wurde bisher nicht genauer behandelt, da sie im Fall eines zeitlich ungepulsten Strahls keine Rolle spielt. Der Vollständigkeit halber wird sie bei der Matrixschreibweise mit angegeben.
} 


$$
=\left(\begin{array}{cccccc}
(x \mid x) & \left(x \mid x^{\prime}\right) & 0 & 0 & 0 & (x \mid \delta) \\
\left(x^{\prime} \mid x\right) & \left(x^{\prime} \mid x^{\prime}\right) & 0 & 0 & 0 & \left(x^{\prime} \mid \delta\right) \\
0 & 0 & (y \mid y) & \left(y \mid y^{\prime}\right) & 0 & 0 \\
0 & 0 & \left(y^{\prime} \mid y\right) & \left(y^{\prime} \mid y^{\prime}\right) & 0 & 0 \\
(l \mid x) & \left(l \mid x^{\prime}\right) & 0 & 0 & 1 & (l \mid \delta) \\
0 & 0 & 0 & 0 & 0 & 1
\end{array}\right) .
$$

Die zweite Schreibweise verdeutlicht, welche Komponenten über die Transformationsmatrix miteinander verknüpft sind. Da die Phasenraumkoordinaten nicht die gleichen Einheiten haben, sind die Matrixelemente einheitenbehaftet. Das Element $\left(x \mid x^{\prime}\right)$ hat z. B. die Einheit $\mathrm{mm} / \mathrm{mrad}$.

Einige Matrixelemente entsprechen direkt bestimmten Eigenschaften optischer Abbildungen, so dass hierfür feststehende Begriffe verwendet werden können. $(x \mid x)$ und $(y \mid y)$ sind die Abbildungsmaßstäbe und $(x \mid \delta)$ entspricht der Ortsdispersion, d.h. der Abhängigkeit des Ortes eines Teilchens von seiner Impulsabweichung.

\subsection{Teilchenstrahl und Emittanz}

In der Regel hat man es bei der Berechnung der Strahlführungselemente nicht mit einzelnen Ionen und ihren Trajektorien zu tun, sondern muss ein Ionenbündel betrachten, das aus einer großen Anzahl von Ionen besteht. Das Ionenbündel wird durch sein Volumen im Phasenraum beschrieben, das als $6 \times 6$ Matrix geschrieben werden kann. Oft kann diese Matrix in Untermatrizen aufgeteilt werden, nämlich dann, wenn die beiden Bewegungsrichtungen als unabhängig voneinander betrachtet werden können.

$$
\sigma=\left(\begin{array}{cccccc}
\sigma_{11} & \sigma_{12} & 0 & 0 & \sigma_{15} & \sigma_{16} \\
\sigma_{21} & \sigma_{22} & 0 & 0 & \sigma_{25} & \sigma_{26} \\
0 & 0 & \sigma_{33} & \sigma_{34} & 0 & 0 \\
0 & 0 & \sigma_{43} & \sigma_{44} & 0 & 0 \\
\sigma_{51} & \sigma_{52} & 0 & 0 & \sigma_{55} & \sigma_{56} \\
\sigma_{61} & \sigma_{62} & 0 & 0 & \sigma_{65} & \sigma_{66}
\end{array}\right)
$$

Die Matrix $\sigma$ ist symmetrisch, d.h. es gilt: $\sigma_{i j}=\sigma_{j i}$. Die Matrix gibt das Phasenraumvolumen an, in dem sich ein Teilchen des Strahls aufhalten kann. Es gilt $x_{\max }=\sqrt{\sigma_{11}}, x_{\max }^{\prime}=\sqrt{\sigma_{22}}, .$. etc. Das heißt, die Diagonalelemente geben die Ausdehnung des Strahls in den verschiedenen Phasenraumkoordinaten an. Die 
Nichtdiagonalelemente sind ein Maß für die Korrelation der verschiedenen Komponenten. Man kann einen dimensionslosen Korrelationsparameter $r_{i j}$ definieren, dessen Wertebereich das Intervall $[-1,1]$ umfasst:

$$
r_{i j}=\frac{\sigma_{i j}}{\sqrt{\sigma_{i i} \sigma_{j j}}} \text {. }
$$

Wenn man die Strahlmatrix in zweidimensionale Untermatrizen aufteilt, lässt sich der Strahl als Ellipse im zweidimensionalen Phasenraum darstellen. Die von der Phasenellipse umrandete Fläche wird als Emittanz bezeichnet.

$$
E_{x}=\pi \sqrt{\operatorname{det} \sigma_{x}}=\pi \sqrt{\sigma_{11} \sigma_{22}-\sigma_{12}^{2}} .
$$

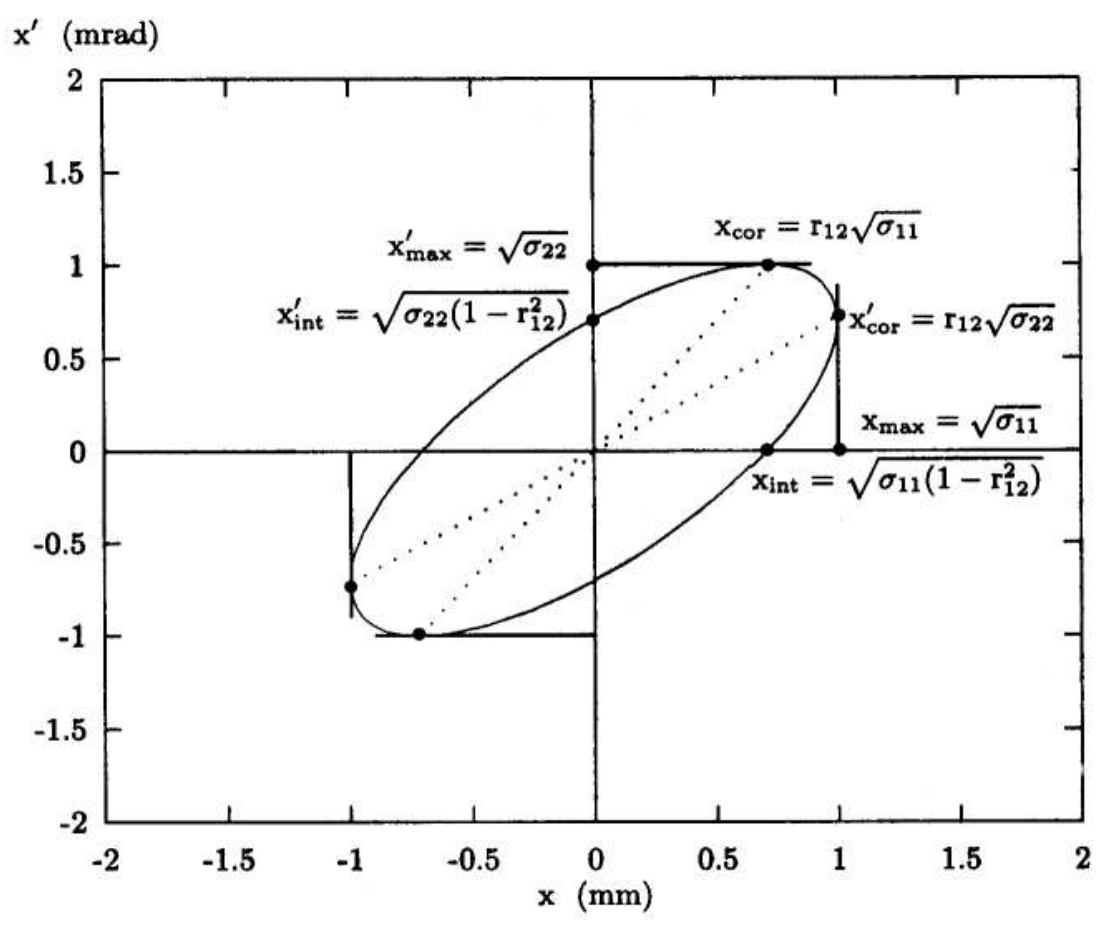

Abbildung 2.2: Phasenellipse [28].

\subsection{Transformation der Phasenraumellipse}

Die Transformation der Strahlmatrix durch ein ionenoptisches System ergibt sich aus der in Abschnitt 2.5 beschriebenen Transfermatrix [28].

$$
\sigma(s)=R(s) \sigma(0) R^{T}(s)
$$


worin $R^{T}(s)$ die transponierte Matrix bezeichnet. Durch diese Transformation ändert sich nur die Form der Phasenellipse, ihre Fläche bleibt konstant. Diese Tatsache wird nach dem Liouvilleschen Theorem gefordert, das aussagt, dass ein Phasenraumvolumen unter Wirkung von konservativen Kräften konstant bleibt [29].

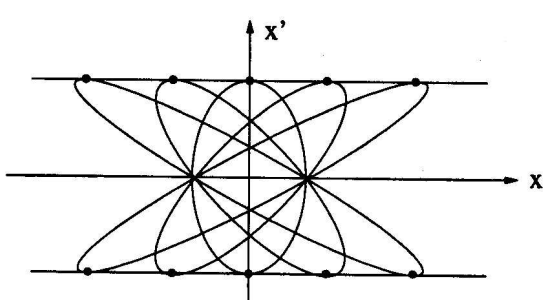

Abbildung 2.3: Transformation der Phasenellipse durch Driftstrecken [28].

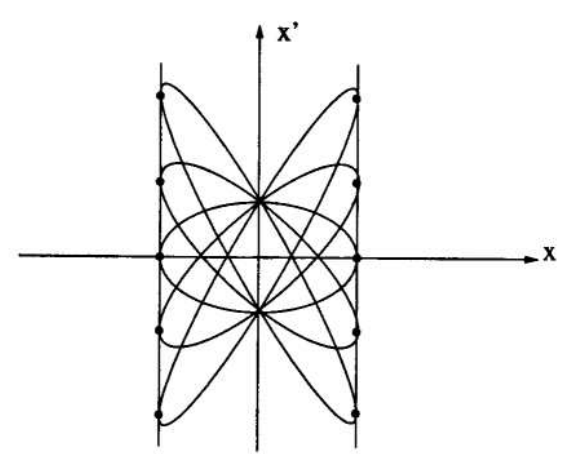

Abbildung 2.4: Transformation der Phasenellipse durch dünne Linsen [28].

Graphisch betrachtet handelt es sich bei den Formänderungen zumeist um Scherungen. Eine Driftstrecke bewirkt z. B. eine Scherung der Strahlellipse in horizontaler Richtung, d.h. die Winkelkoordinate eines jeden Teilchens bleibt konstant, die räumliche Ausdehnung des Strahls und die Korrelationskoeffizienten werden verändert. Eine dünne Linse bewirkt eine vertikale Scherung, d.h. die Ortsabweichung eines jeden Teilchens bleibt konstant. Für Sammellinsen ergibt sich ein negativer Korrelationskoeffizient, für Zerstreuungslinsen ein positiver.

\subsubsection{Dispersive Aufweitung der Phasenraumellipse}

Wenn in einem optischen System die Dispersion nicht null ist, wird die Strahlellipse entlang einer Linie, die durch die Terme $R_{16}$ und $R_{26}$ gegeben ist, aufgeweitet (s. Abb. 2.5. Dies widerspricht nicht dem Liouvilleschen Theorem. Die Darstellung in Abb. 2.5 ist eine Projektion in den zweidimensionalen $\left(x, x^{\prime}\right)$-Raum. Das 6-dimensionale Phasenraumvolumen bleibt konstant. Bei gegebener Impulsbreite lässt sich die Emittanzaufweitung minimieren, wenn die monoenergetischen Strahlellipsen an der Dispersionslinie ausgerichtet sind.

Die dispersive Emittranzaufweitung lässt sich auch quantifizieren, wenn man das Verhältnis der Ellipsenflächen mit und ohne Dispersion oder wahlweise mit und 


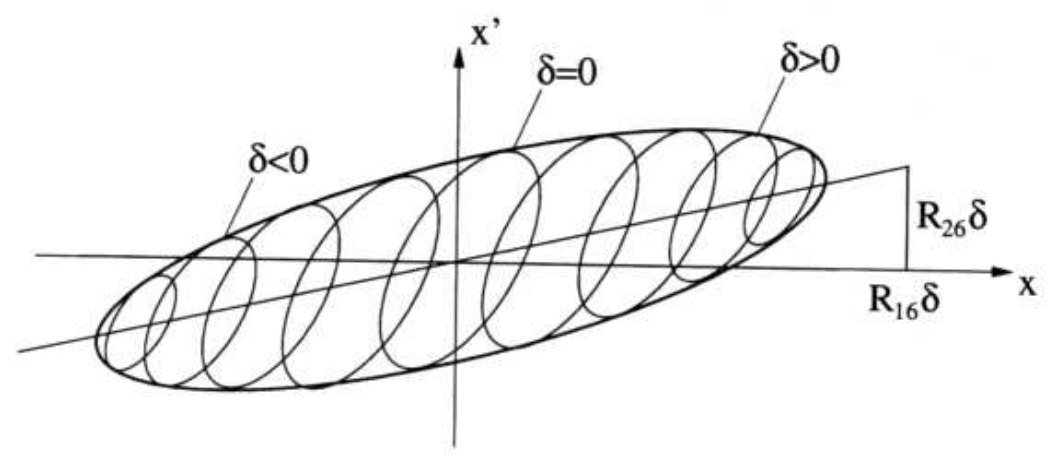

Abbildung 2.5: Dispersive Aufweitung der Phasenellipse [28].

ohne Impulsunsicherheit berechnet. Im Fall von aufrechten Strahlelipsen können die Ausdehnungen des Stahls genommen werden. Man kann dann definieren:

$$
\text { Emittanzaufweitung }=\sqrt{\frac{\sigma_{11}(s) \sigma_{22}(s)}{\sigma_{11}(0) \sigma_{22}(0)}} .
$$

\subsection{Transfermatrizen ausgewählter Elemente}

\subsubsection{Driftstrecke}

Wie aus Abschnitt 2.3 bekannt, sind die Lösungen der DGL für eine Driftstrecke Geraden:

$$
\begin{aligned}
& c_{i}(s)=a_{c} s+b_{c}, \\
& s_{i}(s)=a_{s} s+b_{s} .
\end{aligned}
$$

Die Parameter haben nach 2.13 folgende Werte

$$
\begin{aligned}
& a_{c}=0 \quad, \quad b_{c}=1, \\
& a_{s}=1 \quad, \quad b_{s}=0 \text {. }
\end{aligned}
$$

Daraus ergibt sich an der Stelle $s=L$ (L=Länge der Driftstrecke)

$$
\begin{array}{lll}
c_{i}(L)=1 & , & c_{i}^{\prime}(L)=0, \\
s_{i}(L)=L & s_{i}^{\prime}(L)=1 .
\end{array}
$$


Die komplette 6-dimensionale Transformationsmatrix lautet [28]:

$$
R_{\text {Drift }}=\left(\begin{array}{cccccc}
1 & L & 0 & 0 & 0 & 0 \\
0 & 1 & 0 & 0 & 0 & 0 \\
0 & 0 & 1 & L & 0 & 0 \\
0 & 0 & 0 & 1 & 0 & 0 \\
0 & 0 & 0 & 0 & 1 & L / \gamma^{2} \\
0 & 0 & 0 & 0 & 0 & 1
\end{array}\right)
$$

\subsubsection{Quadrupolmagnet}

Ein Quadrupolmagnet wird charakterisiert durch zwei Parameter, die Länge $L$ und den Gradienten $g$. Ersetzt man den Ausdruck $\frac{q g}{p_{0}}$ aus Gleichung 2.11 durch eine Konstante $k$, so lauten die Lösungen für einen horizontal fokussierenden Quadrupolmagneten:

$$
\begin{aligned}
& c_{x}(s)=A \cos (\sqrt{k} s) s_{x}(s)=B \sin (\sqrt{k} s), \\
& c_{y}(s)=A \cosh (\sqrt{k} s) \quad, \quad s_{y}(s)=B \sinh (\sqrt{k} s) .
\end{aligned}
$$

Aus den Anfangsbedingungen 2.13 ergeben sich:

$$
A=1 \quad, \quad B=\frac{1}{\sqrt{k}}
$$

Die komplette 6-dimensionale Transformationsmatrix lautet [28]:

$$
R_{Q}=\left(\begin{array}{cccccc}
\cos (\sqrt{k} L) & \frac{1}{\sqrt{k}} \sin (\sqrt{k} L) & 0 & 0 & 0 & 0 \\
-\sqrt{k} \sin (\sqrt{k} L) & \cos (\sqrt{k} L) & 0 & 0 & 0 & 0 \\
0 & 0 & \cosh (\sqrt{k} L) & \frac{1}{\sqrt{k}} \sinh (\sqrt{k} L) & 0 & 0 \\
0 & 0 & \sqrt{k} \sinh (\sqrt{k} L) & \cosh (\sqrt{k} L) & 0 & 0 \\
0 & 0 & 0 & 0 & 1 & L / \gamma^{2} \\
0 & 0 & 0 & 0 & 0 & 1
\end{array}\right)
$$

Durch eine geeignete Anordnung von mehreren Quadrupolen in Dubletts oder Tripletts kann man optische Elemente mit fokussierenden Eigenschaften in beiden Richtungen erhalten. 


\subsubsection{Analysiersystem}

Ein System bestehend aus zwei Schlitzen, zwei Driftstrecken und einem $90^{\circ}$ Dipolmagneten mit zwei fokussierenden Kanten wird Analysiersystem genannt, wenn die Parameter folgende Bedingungen erfüllen:

$$
L=2 \rho_{0} \quad, \quad \beta_{1}=\beta_{2}=26,6^{\circ} .
$$

L bezeichnet hierbei die Länge der Driftstrecken vor und hinter dem Dipolmagneten, $\rho_{0}$ den Biegeradius und $\beta_{1}$ und $\beta_{2}$ : die Kanten am Eingang und Ausgang des Dipolmagneten.

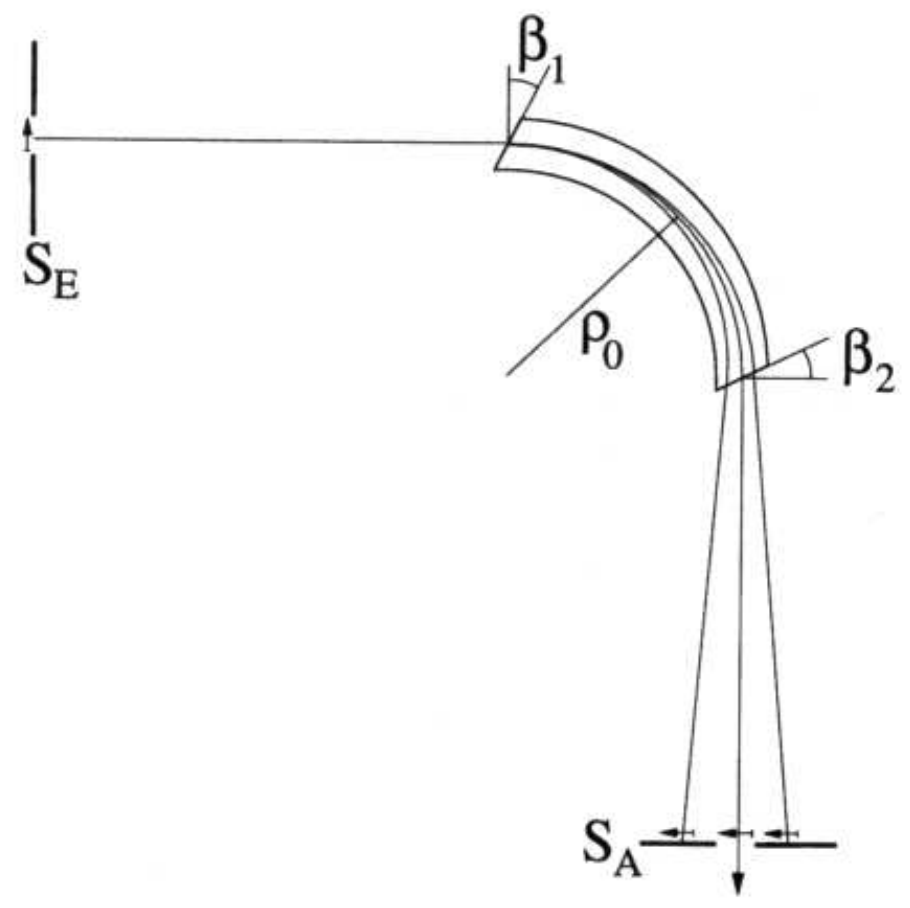

Abbildung 2.6: Analysiersystem [28]. $S_{E}$ und $S_{A}$ bezeichnen die Schlitze am Eingang und Ausgang des Analysiersystems.

Die Abbildungseigenschaften ergeben sich aus der Multiplikation der Transfermatrizen der fünf ionenoptischen Elemente. Ein homogener Ablenkmagnet (Sek- 
tormagnet) besitzt die Transformationsmatrix

$$
R_{\text {Dipol }}=\left(\begin{array}{cccccc}
\cos \alpha & \rho_{0} \sin \alpha & 0 & 0 & 0 & \rho_{0}(1-\cos \alpha) \\
-\frac{1}{\rho_{0}} \sin \alpha & \cos \alpha & 0 & 0 & 0 & \sin \alpha \\
0 & 0 & 1 & L & 0 & 0 \\
0 & 0 & 0 & 1 & 0 & 0 \\
-\sin \alpha & -\rho_{0}(1-\cos \alpha) & 0 & 0 & 1 & \rho_{0}\left[\frac{\alpha}{\gamma^{2}}-\rho_{0}(1-\sin \alpha)\right] \\
0 & 0 & 0 & 0 & 0 & 1
\end{array}\right)
$$

mit dem Ablenkwinkel $\alpha$, der effektiven Länge $L$ und dem Ablenkradius $\rho_{0}=L / \alpha$.

Die Transformationsmatrix spiegelt die Tatsachen wider, dass die Differentialgleichung der abgelenkten (radialen) Koordinate bis auf den inhomogenen Term die gleiche Form hat wie im Fall des fokussierenden Quadrupols und die DGL der nicht abgelenkten (axialen) Koordinate der einer Driftstrecke entspricht.

Die Transformationsmatrix einer Kante eines Dipolmagneten lautet [28]:

$$
R_{\text {Kante }}=\left(\begin{array}{cccccc}
1 & 0 & 0 & 0 & 0 & 0 \\
\frac{1}{\rho_{0}} \tan \beta & 1 & 0 & 0 & 0 & 0 \\
0 & 0 & 1 & 0 & 0 & 0 \\
0 & 0 & -\frac{1}{\rho_{0}} \tan \beta_{\text {eff }} & 1 & 0 & 0 \\
0 & 0 & 0 & 0 & 1 & 0 \\
0 & 0 & 0 & 0 & 0 & 1
\end{array}\right)
$$

Die Vorzeichenkonvention für $\beta$ ist aus der Abbildung $2.6 \mathrm{zu}$ ersehen. Der Wert $\beta_{\text {eff }}$ ist ein leicht von $\beta$ abweichender Winkel, der unter anderem von der Form des Polschuhs abhängt. Man sieht, dass man für $\beta=0$ die Einheitsmatrix erhält.

Wenn die Bedingungen 2.34 erfüllt sind, liefert das System in beiden Richtungen eine umgekehrt reelle Punkt-zu-Punkt Abbildung (s. Kapitel 3). Durch die Dispersion ist es möglich, mit den Schlitzen die Energiebreite eines Strahls zu verringern. Zur übersichtlicheren Darstellung ist die Matrix für nichtrelativistische Teilchen $(\gamma=1)$ und einen Ablenkwinkel von 90 angegeben.

$$
R_{A S}=\left(\begin{array}{cccccc}
-1 & 0 & 0 & 0 & 0 & 4 \rho_{0} \\
-0,75 / \rho_{0} & -1 & 0 & 0 & 0 & 1,5 \\
0 & 0 & -1 & 0 & 0 & 0 \\
0 & 0 & -0,61 / \rho_{0} & -1 & 0 & 0 \\
0 & 0 & 0 & 0 & 1 & 5 \rho_{0} \\
0 & 0 & 0 & 0 & 0 & 1
\end{array}\right)
$$


Das Auflösungsvermögen des Analysiersystems ist gegeben durch [28]:

$$
\left|\frac{R_{16}}{R_{11}}\right|=4 \rho_{0}
$$

\subsection{Transfermatrix 2. Ordnung}

Die Berechnung eines ionenoptischen Systems in 1. Ordnung ist für viele Zwecke ausreichend, und die Transfermatrix 1. Ordnung liefert ein anschauliches Bild der Abbildungseigenschaften. Die Berücksichtigung von Termen höherer Ordnung ist notwendig, wenn ein Teilchenbündel eine große Ausdehnung oder eine große Impulsbreite besitzt. Hierzu werden aus Gleichung 2.7 zusätzlich alle quadratischen Terme der Koordinaten und ihrer Ableitungen berücksichtigt. Die Transformation eines ionenoptischen Elements wird dann beschrieben durch die bereits besprochene Transformationsmatrix 1. Ordnung und einer zusätzlichen Matrix 2. Ordnung. Analog zu Gleichung 2.18 ergibt sich für eine Koordinate eines Teilchens:

$$
x_{i}(s)=\sum_{j=1}^{6} R_{i j}(s) x_{j}(0)+\sum_{k=1}^{6} \sum_{j=1}^{k} T_{i j k}(s) x_{j}(0) x_{k}(0) .
$$

Eine ausführliche Darstellung der Matrixelemente 2.Ordnung ist bei Brown [27] zu finden. Für die vorliegende Arbeit sind Rechnungen von 2.Ordnung nur von untergeordneter Bedeutung, wie im nächsten Kapitel deutlich werden wird. 


\section{Kapitel 3}

\section{Optik des Mikrostrahls}

Die Anforderungen, die an die Ionenoptik des PTB-Mikro-Ionenstrahls gestellt werden, ähneln weitgehend denen von mikrofokussierten Ionenstrahlen, wie sie in der Materialwissenschaft zum Einsatz kommen. Dort werden seit den 70er Jahren mikrofokussierte Ionenstrahlen als Werkzeug zur ortsaufgelösten Elementanalyse verwendet (Nuclear Micro Probes, NMP). Weltweit nimmt die Anzahl solcher Anlagen zu, und verbesserte Geräte erlauben inzwischen eine Ortsauflösung von unter $1 \mu m[30,31]$.

Das Prinzip dieser Anlagen ist, dass ein Ionenstrahl durch ein magnetoptisches System verkleinert auf die zu untersuchende Probe abgebildet wird und dort für das Targetmaterial charakteristische Reaktionen hervorruft. Inzwischen ist eine ganze Reihe von unterschiedlichen Untersuchungsmethoden etabliert. Als Beispiele für die Elementanalyse seien hier PIXE (Particle Induced X-ray Emission) und RBS (Rutherford Back Scattering) genannt [32, 33]. Obwohl dort die Randbedingungen andere sind, können einige apparative Prinzipien in wesentlichen Teilen für den PTB Mikro-Ionenstrahl übernommen werden.

\subsection{Abbildung in 1. Ordnung}

Wie bereits in Abschnitt 2.5 gezeigt, werden die Abbildungseigenschaften eines ionenoptischen Systems bestimmt durch die Anordnung von ionenoptischen Elementen (Magnete, Driftstrecken, etc.) und sind somit unabhängig von den Eigenschaften des Eingangsstrahls. Die Strahleigenschaften nach der Abbildung hingegen hängen von den Abbildungseigenschaften der Ionenoptik und vom Eingangstrahl ab.

Zur Mikrofokussierung wählt man in diesem Fall eine Punkt-zu-Punkt Abbildung, da der Eingangsstrahl, der durch Objekt- und Aperturblenden definiert wird, 
zumeist kleine Orts- und große Winkelausdehnungen besitzt. Die Bedingung für eine Punkt-zu-Punkt Abbildung lautet:

$$
\begin{aligned}
& R_{12}=0, \\
& R_{34}=0,
\end{aligned}
$$

d.h. die Ausdehnung des Strahls am Fokuspunkt hängt in 1. Ordnung nicht von der Eingangsdivergenz ab.

Der wichtigste Parameter der ionenoptischen Abbildung ist der Abbildungsmaßstab. Der Abbildungsmaßstab ist das Verhältnis von Bild- zu Objektgröße und ist in den beiden transversalen Koordinaten durch die Matrixelemente $R_{11}$ und $R_{33}$ gegeben. Bei der Mikrofokussierung ist ein sehr kleiner Abbildungsmaßstab erwünscht. Die Verkleinerung oder Demagnifikation ist gegeben durch:

$$
\left|\frac{1}{R_{11}}\right|=D_{x} \quad, \quad\left|\frac{1}{R_{33}}\right|=D_{y} .
$$

Da die Größe der Phasenellipse nach dem Liouvilleschen Theorem nicht geändert werden kann [29], muss die Winkelausdehnung sich um denselben Faktor vergrößern.

$$
\left|R_{22}\right| \geq D_{x} \quad, \quad\left|R_{44}\right| \geq D_{y}
$$

Bei der Fokussierung mit Quadrupolmagneten sind die Demagnifikationen in $x$ und $y$ im allgemeinen unterschiedlich. Je nach Aufbau der Strahlführung werden in existierenden Anlagen Werte zwischen 20 und 200 erreicht. Oftmals wird durch eine Anordnung von Linsen um Symmetriepunkte versucht, Magnete mit identischer Anregung zu verwenden und so die Anzahl der zu justierenden Parameter $\mathrm{zu}$ verringern.

\subsection{Intrinsische Aberrationen}

Bei der Mikrofokussierung wird die Ortsauflösung oft von Effekten höherer Ordnung, den Aberrationen, limitiert. Dies liegt daran, dass die Ausdehnung des Strahls im Fokus sehr klein ist und deshalb die Effekte der höheren Ordnung bemerkbar werden, die bei größeren Strahldimensionen keine Rolle spielen. 
Den wichtigsten Beitrag liefern zumeist die chromatischen Aberrationen, das sind die Terme $T_{i j 6}$, insbesondere $T_{126}=\left(x \mid x^{\prime} \delta\right)$ und $T_{346}=\left(y \mid y^{\prime} \delta\right)$. Diese Terme werden durch die ionenoptische Abbildung festgelegt. Man versucht, durch besondere, in der Regel symmetrische Anordnungen die chromatischen Aberrationen, das heißt die Größe der Koeffizienten, zu minimieren [31]. Ein anderer Ansatz ist die Verwendung von elektrostatischen Linsen, deren chromatische Aberrationen das umgekehrte Vorzeichen wie magnetische Linsen haben, so dass man durch eine Kombination eine Kompensation erreichen kann [34].

Da die Auswirkungen der intrinsischen Aberrationen auf die Strahleigenschaften mit steigendem Emittanzvolumen zunehmen, werden sie durch eine Verringerung der Eingangsemittanz reduziert. Die hiermit verbundene Reduzierung des Strahlstroms ist jedoch bei vielen Experimenten nicht tolerierbar. Um einen genügend großen Strahlstrom zu erreichen, arbeiten viele Institute, die mikrofokussierte Ionenstrahlen betreiben, an verbesserten Ionenquellen. Der Strom je Phasenraumvolumen, als Brillanz einer Ionenquelle bezeichnet, wird zunehmend zum Qualitätsmerkmal und zum Ansatzpunkt für weitere Verbesserungen in der Ortsauflösung von Nuklearen Mikrostrahlen [31].

Für Mikrostrahlen in der Materialforschung spielt die Minimierung der Aberrationen die dominante Rolle in der Ionenoptik, und die Strahlführungen werden im Hinblick auf diesen Gesichtspunkt optimiert. Der Aufbau des Mikro-Ionenstrahls der PTB lässt wenig Spielraum hierfür, da der Strahlverlauf einen Dipolmagneten mit fixierten Abbildungseigenschaften enthält. Hier stehen andere Parameter im Vordergrund.

Für radiobiologische Experimente mit einzelnen Ionen ist es notwendig, den Strahlstrom stark zu reduzieren, so dass die Aberrationen wegen des kleinen Emittanzvolumens nicht ins Gewicht fallen. Im Normalbetrieb liefern die Beschleuniger ca. $1 \mu$ A Strahlstrom. Um auf 1000 Ionen je Sekunde zu kommen, muss der Strom etwa um einen Faktor $10^{10}$ reduziert werden. Das heißt, dass in beiden Richtungen das Emittanzvolumen von ca. $5 \mathrm{~mm}$ mrad auf $50 \mu \mathrm{m} \mu \mathrm{rad}$ reduziert wird. Bei Objektgrößen von $1 \mu \mathrm{m}$ bis $5 \mu \mathrm{m}$ ergibt dies eine Eingangsdivergenz von $10 \mu \mathrm{rad}$ bis $50 \mu \mathrm{rad}$. Wie die Berechnung der Strahlführung in Abschnitt 3.4 .2 zeigen wird, ist bei einer geeigneten Optik nicht zu erwarten, dass die intrinsischen Aberrationen einen messbaren Effekt verursachen. 


\subsection{Parasitäre Aberrationen}

Die intrinsischen Aberrationen haben ihre Ursache in den nichtlinearen Termen der Bahngleichung 2.7 und sind somit physikalischen Ursprungs. Andere Effekte werden durch Limitierung der Genauigkeit und Mängel der Technik hervorgerufen. Diese werden unter dem Begriff parasitäre Aberrationen zusammengefasst. Die wichtigsten sind:

a. unerwünschte Multipolanteile in den Magnetfeldern der Strahlführungselemente

b. mechanische Fehljustierungen der Strahlführungselemente

c. magnetische Streufelder durch elektrische Geräte oder das Erdmagnetfeld

d. Vibrationen des Targets oder der Strahlführungselemente

e. Streuung des Teilchenstrahls am Restgas in der Vakuumkammer

f. Streuung im Material des Vakuumfensters

Einige dieser Effekte, wie Vibrationen und störende Magnetfelder, sind a priori nicht genau berechenbar, sondern nur anhand von Erfahrungswerten grob abschätzbar. Durch geeignete apparative Anordnungen und Vorkehrungen kann man versuchen, den Einfluss der parasitären Aberrationen gering zu halten.

Zu Punkt a: Parasitäre Multipolanteile spielen insbesondere bei Quadrupolmagneten eine Rolle. Sie nehmen von der Achse nach außen hin zu und erreichen ein Maximum in der Nähe des Polschuhs. Durch präzise Fabrikation und Montage des Magneten lassen sie sich verringern. Für Experimente mit hohem Strom muss ein Kompromiss gefunden werden zwischen der Notwendigkeit, eine kleine Magnetapertur zu verwenden, um eine große Fokussierstärke zu erreichen, und den Aberrationen, die durch die Multipolanteile entstehen.

Für die radiobiologischen Experimente mit einzelnen Ionen wird die Eingangsdivergenz zur Strahlstromreduzierung stark eingeschränkt. Deshalb befinden sich alle Strahlteilchen selbst nach einer langen Driftstrecke in der Nähe der Sollbahn, so dass parasitäre Multipolanteile kein Problem darstellen sollten. Der Hersteller 1

\footnotetext{
${ }^{1}$ Danfysik A/S, 4040 Jyllinge, Dänemark.
} 


\begin{tabular}{|l|c|c|}
\hline \multirow{2}{*}{ Harmonische } & \multicolumn{2}{|c|}{ Feldstärke relativ zur Hauptkomponente } \\
& 9 mm Achsabstand & 3,5 mm Achsabstand \\
\hline 3 & $0,1 \%$ & $0,015 \%$ \\
4 & $0,03 \%$ & $0,002 \%$ \\
5 & $0,03 \%$ & $0,0004 \%$ \\
6 & $0,2 \%$ & $0,002 \%$ \\
10 & $0,1 \%$ & $0,00002 \%$ \\
\hline
\end{tabular}

Tabelle 3.1: Multipolverunreinigungen der Quadrupole.

des mikrofokussierenden Quadrupoldubletts gibt die Werte aus Tabelle 3.1 für die Multipolverunreinigungen an.

Zu Punkt b: Die Mikrofokussierung stellt hohe Anforderungen an die mechanische Genauigkeit, mit der die ionenoptischen Komponenten justiert werden. Die Genauigkeit sollte besser als $0,1 \mathrm{~mm}$ in den Translationen und 0,1 mrad in den Verkippungen und Rotationen betragen [31]. Das mikrofokussierende Quadrupoldublett wurde deshalb auf einem Rahmen montiert, der die Justierung mit Feingewindeschrauben M8x1 erlaubt. Zudem werden alle Koordinaten von Messuhren mit einer Genauigkeit von $10 \mu \mathrm{m}$ angezeigt. Die anderen ionenoptischen Komponenten wie das erste Quadrupoldublett, der Dipol und die Schlitze sind ebenfalls mit Justiermechaniken ausgestattet und werden per optischem Verfahren eingemessen.

Zu Punkt c: Wenn im Bereich der Strahlführung unerwünschte magnetische Felder von elektrischen Geräten auftreten, die eine Störung des Strahls verursachen, kann man das Strahlrohr mit Mumetall ${ }^{\circledR 2} 2$ dagegen abschirmen oder versuchen, die Quelle das Störfeldes zu beseitigen.

Des Weiteren kann das Erdmagnetfeld einen Einfluss auf den Strahl haben. Die Stärke des Effektes soll im Folgenden grob abgeschätzt werden. In Deutschland beträgt die magnetische Induktion des Erdmagnetfeldes parallel und senkrecht zur Erdoberfläche ca. [35]:

$$
\begin{aligned}
& B_{\|}=20 \mu T, \\
& B_{\perp}=40 \mu T .
\end{aligned}
$$

Für ein Proton von $3 \mathrm{MeV}$ ergeben sich Biegeradien von $r_{\|}=1250 \mathrm{~m}$ und $r_{\perp}=625 \mathrm{~m}$. Da das Strahlrohr des Mikro-Ionenstrahls fast genau nach Norden

\footnotetext{
${ }^{2}$ Mumetall ist eine antimagnetische Schutzlegierung $(\mathrm{Ni}(77) \mathrm{Fe}(14) \mathrm{Cu}(5) \mathrm{Mo}(4))$.
} 
ausgerichtet ist, kann zur Vereinfachung angenommen werden, dass im horizontalen Teil nur die senkrechte Magnetfeldkomponente wirksam ist, und im vertikalen Teil nur die parallele. Für die Driftstrecke von der Lochblende bis zum Divergenzschlitz von ca. $2 \mathrm{~m}$ ergibt sich eine Abweichung von $0,3 \mathrm{~mm}$ und eine Richtungsabweichung von 0,15 mrad. Zur Zeit kann der Einfluss des Erdmagnetfeldes nicht von anderen Effekten wie mechanischen Fehljustierungen separiert werden. Wenn diese beseitigt sind, kann es sinnvoll werden, den Effekt des Erdmagnetfeldes zu veringern. Neben der Abschirmung mit Mumetal ${ }^{\circledR}$ kann die Ablenkung durch das Erdmagnetfeld durch Korrekturmagnete (Steerer) kompensiert werden.

Zu Punkt d: Vibrationen spielen bei Mikrostrahlen zur Materialforschung eine große Rolle, da die Proben in einem Manipulator im Vakuum angebracht sind, der in der Regel anfällig für Vibrationen ist. Durch solide Betonfundamente und einen schwingungsarmen Aufbau der Strahlführung versucht man die Anregung von Schwingungen zu vermeiden. Gegenüber der Vibration des Targets sind die Vibrationen der Strahlführungselemente zu vernachlässigen. Eine transversale Schwingung der Objektschlitze wird z.B. durch die verkleinerte Abbildung verringert, und Schwingungen der Magnete wirken sich nur in Form einer Erhöhung der intrinsischen Aberrationen aus.

Da die Experimentierhalle des PTB Ionenbeschleunigers im Erdgeschoss einen Boden aus Metallgitter hat, der keine ausreichende Vibrationsdämpfung zulässt, musste der Strahl mit einem Dipolmagneten nach unten geführt werden. Die zu bestrahlenden Proben befinden sich dort auf einem vibrationsarmen Tisch, der fest mit dem Hallenboden verbunden ist (vgl. Abbildung 5.1).

Zu Punkt e: Der gemessene Druck im Strahlführungssystem des MikroIonenstrahls beträgt ca. $10^{-4} \mathrm{~Pa}$. Die mittlere freie Weglänge eines Ions beträgt [4]:

$$
\lambda=\frac{1}{\sqrt{2}} \frac{k T}{\sigma_{0} p},
$$

$$
\begin{array}{llll}
\operatorname{mit}: & k & : & \text { Bolzmannkonstante } \\
& T & : & \text { Temperatur } \\
& : & \text { Gasdruck } \\
& \sigma_{0} & : & \text { totaler Wirkungsquerschnitt der Gasteilchen }
\end{array}
$$


Wenn man zur Vereinfachung annimmt, dass der totale Wirkungsquerschnitt durch die Atomquerschnittsfläche von Stickstoff gegeben ist (Atomradius $75 \mathrm{pm}$ [36]), lässt sich hieraus eine mittlere freie Weglänge von ca. 1,5 km abschätzen. Bei einer Gesamtlänge der Abbildungsstrecke von ca. $10 \mathrm{~m}$ sollte daher der Anteil der mit Restgas wechselwirkenden Ionen bei ca. 1\% liegen. Es ist aber zu beachten, dass der Druck an verschiedenen Stellen des Strahlführungssystems durch Lecks z.B. unmittelbar am Vakuumfenster unter Umständen deutlich höher sein kann.

Zu Punkt f: Da Zellen im Vakuum nicht überlebensfähig sind, ist die Streuung der Ionen im Material des Vakuumfensters unvermeidlich. Durch geeignete Maßnahmen muss sie möglichst gering gehalten werden. In Abschnitt 5.3 wird dies ausführlich behandelt.

\subsection{Berechnung der Abbildungseigenschaften}

Die Berechnung der Strahloptik erfolgte mit dem Programm TRANSPORT [37]. Das Programm ist beim Fermilab per Datentransfer über das Internet frei verfügbar ${ }^{3}$. Es beruht auf der Matrixmethode und berechnet Transformationsmatrizen von ionenoptischen Systemen bis zur 3. Ordnung. Neben der Berechnung der Abbildungseigenschaften von festgelegten Komponenten, erlaubt das Programm, Parameter wie z.B. die Position oder die Feldstärke von Magneten anzupassen, um bestimmte Abbildungs- oder Strahleigenschaften zu erreichen (fitten).

Zur Überprüfung der mit TRANSPORT erhaltenen Ergebnisse wurde zusätzlich das Programm TURTLE [38] verwendet. Dieses verfolgt den Weg einzelner Teilchen durch die komplette Strahlführung durch Lösung der entsprechenden Differentialgleichungen (ray tracing). Durch die Variation der Anfangsbedingungen im Rahmen der vorgegebenen Eingangsemittanz kann das resultierende Emittanzvolumen am Fokuspunkt dargestellt werden.

Zusätzlich zu den Bedingungen der Punkt-zu-Punkt Abbildung (Gleichung 3.1) muss die Ortsdispersions am Fokuspunkt minimiert werden:

$$
R_{16}=0,
$$

\footnotetext{
${ }^{3}$ http://www.fnal.gov/fermitools
} 
da diese durch die Verwendung eines Dipols im Allgemeinen nicht null ist.

Außerdem soll eine verkleinerte Darstellung des Objekts erreicht werden:

$$
D_{x}>1, \quad D_{y}>1
$$

Da die Gesamtlänge der Abbildungsstrecke durch die baulichen Gegebenheiten der Experimentierhalle auf ca. $10 \mathrm{~m}$ limitiert ist und der $90^{\circ}$-Magnet festgelegte Abbildungseigenschaften hat, bleiben als freie Parameter nur die Positionen und die Magnetfeldstärken der Quadrupole. Die einfachste doppelfokussierende Anordnung von Quadrupolen ist ein Dublett. Zur Verkleinerung ist ein Quadrupoldublett möglichst dicht vor dem Experimentierort notwendig. Es zeigt sich, dass die drei Bedingungen aus 3.1 und 3.6 durch eine geeignete Wahl der Magnetfelder an dieser Stelle nicht gleichzeitig erfüllt werden können. Im horizontalen Teil wurde deshalb ein zweites Quadrupoldublett installiert.

Zur einfacheren Handhabung wird die Abbildungsstrecke in zwei Abschnitte unterteilt, deren Abbildungseigenschaften separat berechnet werden. Der erste Abschnitt geht von der Objektblende bis zum Ende des Analysiersystems (vgl. Abb. 1.4 und 2.6), d.h. bis 1,50 $\mathrm{m}$ hinter dem Dipolmagneten (Schlitz 43). Er beinhaltet das Analysiersystem mit seinen unveränderlichen Abbildungseigenschaften sowie das horizontale Dublett mit den Driftstrecken davor und dahinter. Der zweite Teil enthält den unteren, vertikalen Teil der Strahlführung, der aus einer langen Driftstrecke, dem mikrofokussierenden Dublett und einer kurzen Driftstrecke, der Bildweite, besteht. Es gilt:

$$
R(\text { ges })=R(\text { ver }) R(\text { hor })
$$

\subsubsection{Kompensation der Dispersion}

Durch Multiplikation lässt sich leicht zeigen, dass die Matrixelemente $R_{12}($ ges $)$ und $R_{16}($ ges $)$ durch folgende Gleichungen gegeben sind:

$$
R_{12}(\text { ges })=R_{11}(\text { ver }) R_{12}(\text { hor })+R_{12}(\text { ver }) R_{22}(\text { hor }),
$$

und

$$
R_{16}(\text { ges })=R_{11}(\text { ver }) R_{16}(\text { hor })+R_{12}(\text { ver }) R_{26}(\text { hor })
$$


da die Abbildung im vertikalen Teil dispersionsfrei ist $\left(R_{16}(\right.$ ver $)=R_{26}($ ver $\left.)=0\right)$. Nach Gleichung 2.37 ergibt sich für die Position am Ausgangsschlitz des Analysiersystems (Schlitz 43)

$$
\begin{aligned}
R_{16}(\text { hor }) & =3 \frac{\mathrm{mm}}{0 / 00}, \\
R_{26}(\text { hor }) & =1,5 \frac{\mathrm{mrad}}{\% / 00} .
\end{aligned}
$$

Mit diesen Werten folgt aus Gleichung 3.10 und 3.6 :

$$
R_{11}(\text { ver })=-\frac{1}{2} R_{12}(\text { ver }) \frac{m r a d}{m m} .
$$

Diese Bedingung betrifft nur die Abbildungseigenschaften des Quadrupoldoubletts im vertikalen Abschnitt. Da mit dessen beiden Magnetströmen zwei freie Parameter zur Verfügung stehen, lassen sich verschiedene Wertepaare für die Magnetströme finden, mit denen sich diese Bedingung erfüllen lässt.

Mit dem Ergebnis aus 3.12 folgt aus 3.9 für die Transformationsmatrix des horizontalen Teils:

$$
R_{12}(\text { hor })=2 R_{22}(\text { hor }) \frac{m r a d}{m m} .
$$

Diese Bedingung betrifft nur die Abbildungseigenschaften des Quadupoldubletts im horizontalen Abschnitt. Auch hierfür können viele Wertepaare der Magnetfeldstärken gefunden werden.

Die Abbildung 3.1 veranschaulicht das Prinzip der so erhaltenen Abbildung in der radialen Richtung. Die dispersiv aufgeweitete Strahlellipse lässt sich durch eine rückwärtige Driftstrecke der Länge $R_{16}(h o r) / R_{26}(h o r)$ in eine aufrechte Strahlellipse transformieren. Dieser Punkt liegt $2 m$ über dem Ausgang des Dipolmagneten und wird im Folgenden als virtuelles Zwischenbild bezeichnet. Das virtuelle Zwischenbild wird durch die Driftstrecke und den unteren Teil des optischen Systems mittels einer Punkt-zu-Punkt Abbildung auf den Experimentierort fokussiert. Der obere Teil der Strahlführung wird so eingestellt, dass das Objekt mit einer Punkt-zu-Punkt Abbildung auf das virtuelle Zwischenbild abgebildet wird. Insgesamt ergibt sich hieraus eine ortsdispersionsfreie Punkt-zu-Punkt Abbildung. Zusätzlich ist die dispersive Aufweitung des Strahls minimiert.

Wie in der Abbildung 3.1 angedeutet ist, existiert auch ein reelles Zwischenbild. Dieses ist chromatisch, d.h. am Zwischenbild verschwinden weder Orts- noch Winkeldispersion, und es befindet sich genau in der Mitte des Dipolmagneten. Die 


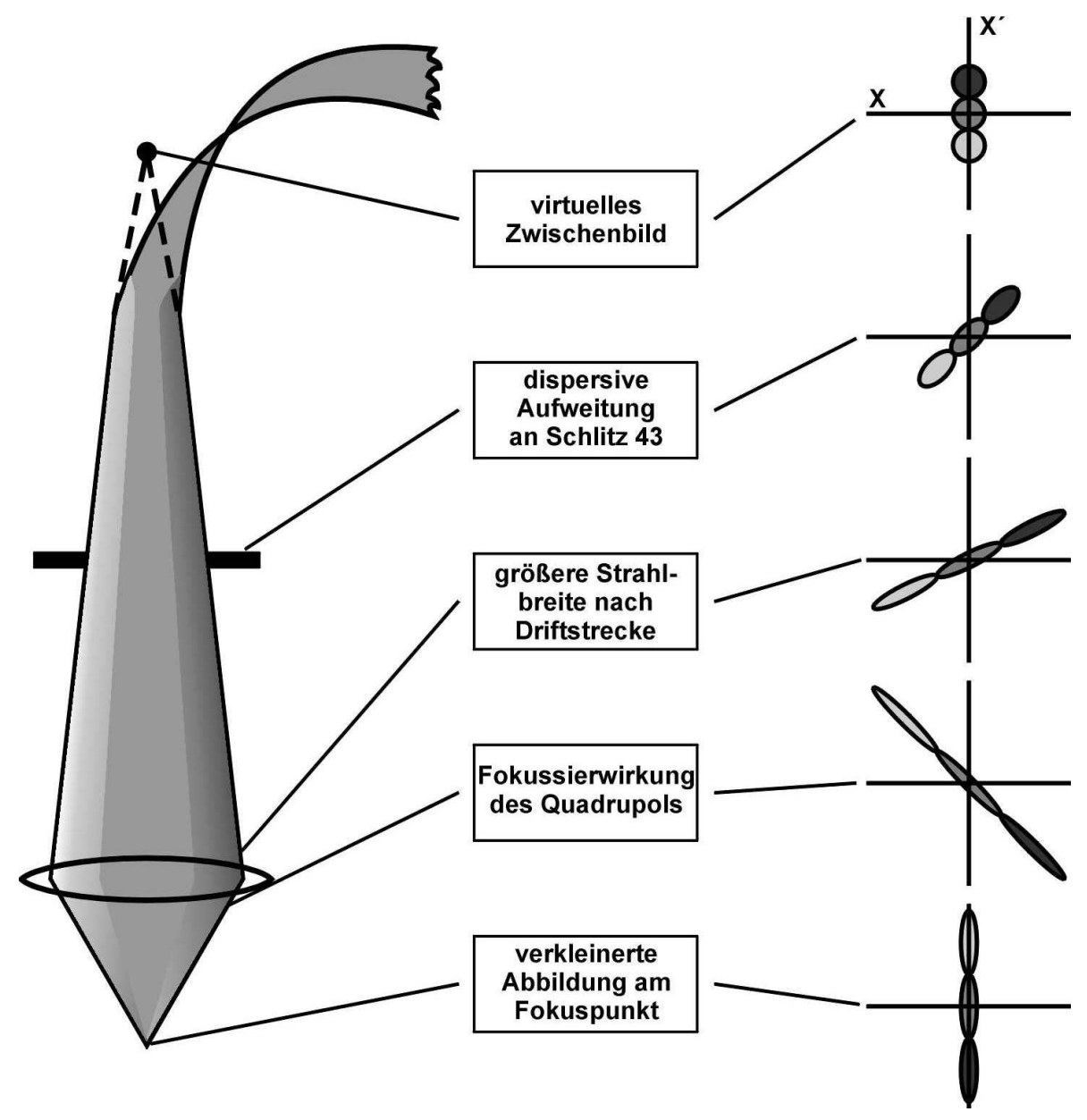

Abbildung 3.1: Prinzip der ortsdispersionsfreien Abbildung. Die drei Ellipsen stellen den Strahl mit Sollimpuls $p_{0}$ und mit leicht geändertem Impuls $p_{0} \pm \delta p$ dar.

ortsdispersionsfreie Abbildung am Fokuspunkt ist nur bei Existenz eines Zwischenbildes an dieser Stelle möglich. Mathematisch ist die Existenz dieses Zwischenbildes äquivalent mit der Ausrichtung der Strahlellipse entlang der Dispersionslinie (vgl. Abschnitt 2.7.1), wie sich durch Matrizenmultiplikation leicht zeigen lässt. Die Abbildungsmaßstäbe vom reellen bzw. vom virtuellen Zwischenbild bis zum Fokuspunkt sind etwa gleichgroß.

\subsubsection{Optische Parameter des Mikro-Ionenstrahls}

Aufbauend auf den Überlegungen aus dem vorhergehenden Abschnitt wurden verschiedene Rechnungen durchgeführt, bei denen die Polung der Magnete verändert 
wurde. Es zeigte sich, dass die gewünschten Eigenschaften nur mit einer symmetrischen Anordnung zu erfüllen sind. Die Magnetanordnungen sind nach den Fokussierungseigenschaften der einzelnen Quadrupolmagnete in der radialen Richtung benannt. Die Abkürzung DFFD bedeutet, dass der erste und der vierte Quadrupol in radialer Richtung defokussierend sind, und der zweite und dritte fokussierend.

Die Ergebnisse der Berechnungen sind in Tabelle 3.2 zusammengestellt. Die Bedingungen 3.1 und 3.6 können durch mehrere Einstellungen erfüllt werden. Da sich zwei der Bedingungen 3.1 und 3.6 auf die radiale Richtung beziehen, und mit den Magnetströmen vier Parameter zur Verfügung stehen, hat man in der axialen Richtung einen Freiheitsgrad übrig. Hier wurden unterschiedliche Verkleinerungen $D_{y}$ gewählt. Des Weiteren sind auch die resultierenden Strahlfleckgrößen und Divergenzen im Fokuspunkt aufgelistet. Hierbei wurde ein Ionenstrahl von $3 \mathrm{MeV}$ Protonen mit $\sqrt{\sigma_{11}(0)}=\sqrt{\sigma_{33}(0)}=5 \mu \mathrm{m}, \sqrt{\sigma_{22}(0)}=\sqrt{\sigma_{44}(0)}=100 \mu \mathrm{rad}$ und $\delta=0,1 \%$ angenommen.

\begin{tabular}{|l||r|r|r||r|r|r|}
\hline \multicolumn{1}{|l||}{ Konfiguration } & \multicolumn{3}{|c||}{ DFFD } & \multicolumn{3}{c|}{ FDDF } \\
\hline$\left|D_{y}\right|$ & 10 & 20 & 50 & 10 & 20 & 50 \\
\hline B1 (kG) & $-0,78696$ & $-0,81617$ & $-0,90253$ & 0,83535 & 0,88944 & 1,01434 \\
B2 $(\mathrm{kG})$ & 0,71569 & 0,72604 & 0,75530 & $-1,00038$ & $-1,18011$ & $-1,70351$ \\
B3 $(\mathrm{kG})$ & 1,01518 & 1,01411 & 1,01346 & $-1,07664$ & $-1,06176$ & $-1,05268$ \\
$\mathrm{~B} 4(\mathrm{kG})$ & $-1,79574$ & $-1,78805$ & $-1,78344$ & 1,79765 & 1,79160 & 1,78788 \\
\hline$R_{11}$ & 0,13121 & 0,12886 & 0,10097 & 0,08272 & 0,09123 & 0,11617 \\
$R_{33}$ & $-0,10000$ & $-0,05000$ & $-0,02000$ & $-0,10000$ & $-0,05000$ & $-0,02000$ \\
\hline$T_{126}(\mu \mathrm{m} / \mathrm{mrad} \% 00)$ & $-16,09$ & $-16,33$ & $-17,17$ & $-3,57$ & $-3,36$ & $-3,01$ \\
$T_{346}(\mu \mathrm{m} / \mathrm{mrad} \% 00)$ & 5,00 & 7,19 & 15,31 & 67,68 & 92,64 & 182,0 \\
\hline$\sqrt{\sigma_{11}(\mathrm{~s})}(\mu \mathrm{m})$ & 0,707 & 0,697 & 0,672 & 0,460 & 0,498 & 0,637 \\
$\sqrt{\sigma_{22}(\mathrm{~s})}(\mu \mathrm{rad})$ & 1113 & 1124 & 1151 & 5581 & 5553 & 6149 \\
$\sqrt{\sigma_{33}(\mathrm{~s})}(\mu \mathrm{m})$ & 0,502 & 0,260 & 0,183 & 0,856 & 0,984 & 1,887 \\
$\sqrt{\sigma_{44}(\mathrm{~s})(\mu \mathrm{rad})}$ & 1021 & 2012 & 5011 & 1000 & 2000 & 5001 \\
\hline
\end{tabular}

Tabelle 3.2: Parameter verschiedener Optiken. B1 - B4 bezeichnen die Magnetfeldstärken der vier Quadrupolmagnete. Die Matrixelemente $R_{12}, R_{34}$ und $R_{16}$ sind nicht aufgeführt, da sie null sind. Die Strahlparameter $\left(\sigma_{i i}\right)$ wurden in der 2. Ordnung berechnet und beinhalten somit die intrinsischen Aberrationen.

Die Ergebnisse zeigen, dass die Bedingungen für die ortsdispersionsfreie Punktzu-Punkt Abbildung exakt erfüllt werden können. In axialer Richtung kann eine 
Verkleinerung von 50 und mehr erreicht werden, während diese in radialer Richtung auf 6 bis 10 begrenzt ist. Bei sehr kleinen Gegenstandsweiten kann die Verkleinerung allerdings nicht voll genutzt werden, da die Aberrationen dann merkliche Beiträge zur Strahlbreite liefern.

Der veränderte Abbildungsmaßstab in axialer Richtung wird im Wesentlichen durch eine stärkere Fokussierung im horizontalen Dublett erreicht. Durch die kürzere Bildweite im horizontalen Teil wird eine etwas längere Gegenstandsweite für das mikrofokussierende Dublett verursacht, so dass dessen Magnetfelder bei zunehmender Verkleinerung etwas schwächer werden. In der radialen Richtung bleibt die Position des virtuellen Zwischenbildes fixiert, und der Abbildungsmaßstab ändert sich nur geringfügig.

Nach den Berechnungen ist die DFFD-Abbildung unproblematischer, da hier die Aberrationen geringer sind und die dispersive Aufweitung in radialer Richtung wesentlich kleiner ist (vgl. Abschnitt 2.7.1). Es wurde daher diese Optik gewählt und immer mit einer axialen Demagnifikation von ca. 20 gearbeitet, weil der Strahl dann eine axiale Taille am Schlitz 42 besitzt. Dies ist vorteilhaft, weil diese Schlitzweite für die Effizienz des Deflektors gering sein muss (s. Abschnitt 4.2). Die experimentellen Resultate in Kapitel 6 wurden daher alle mit der DFFDOptik und einer axialen Verkleinerung von 20 erzielt.

Die Berechnungen zeigt, dass mit der gewählten Konzeption eine geeignete Abbildung eingestellt werden kann. Insbesondere ist es möglich, die Ortsdispersion am Fokuspunkt exakt auf null zu bringen. Im Experiment können solche idealen Bedingungen kaum erreicht werden. Schon allein die Einstellgenauigkeit der Stromstärke von ca. $5 \mathrm{~mA}$ für das mikrofokussierende Dublett sowie $10 \mathrm{~mA}$ für das horizontale Dublett reichen nicht aus, um die errechneten Magnetfelder exakt einzustellen. Zudem ist das Kriterium für die experimentelle Einstellung der Magnetströme im Wesentlichen der Strahlfleck am Fokuspunkt. Dieser hängt von allen oben angegebenen Parametern ab, wobei deren Einflüsse nicht voneinander separiert werden können.

Die Werte, die experimentell für eine optimale Strahlfokussierung gefunden wurden, stimmen mit einer hohen Genauigkeit ( $<1 \%$ Abweichung) mit den berechneten überein. Es ist jedoch zu beachten, dass im Falle der Quadrupolmagnete die magnetische Induktion $B$ nicht gemessen werden kann, und dass aufgrund der Sättigung und der Hysterese keine exakt lineare Beziehung zwischen Strom und Feld eines Magneten herrscht. Dies führt dazu, dass die Strahloptik stets experimentell optimiert werden muss. 


\section{Kapitel 4}

\section{Elemente der Strahlführung}

In diesem Kapitel werden die Merkmale der verschiedenen Strahlführungselemente, die bereits in Abschnitt 1.4 erwähnt wurden, ausführlich beschrieben. Eine detaillierte Darstellung des kompletten Strahlführungssystems ist in Abbildung 4.1 gegeben.

\subsection{Objekt- und Aperturblende}

Die Objektblende befindet sich unmittelbar hinter dem Schaltmagneten noch außerhalb der Experimentierhalle (vgl. Abb. 1.2). Hierdurch wird die Länge der Abbildungsstrecke maximiert, wodurch eine höhere Verkleinerung möglich wird. Außerdem wird der Strahlstrom schon außerhalb der Experimentierhalle stark eingeschränkt, was zu einer Reduzierung der Aktivierung der Strahlführungselemente durch die hochenergetischen Ionen führt. Deshalb ist dem Experimentator ein ungefährdeter, ständiger Zugang zur Experimentierhalle ermöglicht. Das Objekt besteht aus einer Kupferplatte mit Bohrungen von $1 \mathrm{~mm}$ Durchmesser, über die eine Metallfolie gelegt ist, die ihrerseits in der Mitte der Bohrungen Löcher von $1 \mu \mathrm{m}$ bis $30 \mu \mathrm{m}$ Durchmesser besitzt (s. Abbildung 4.2). Die Mikrolöcher in der Metallfolie wurden mit einem Fokussierten Ionenstrahl (FIB) am Institut für Angewandte Festkörperphysik der Universität Bochum hergestellt [39]. Aufgrund der ungleichen Abbildungsmaßstäbe werden Mikrobohrungen mit elliptischer Form verwenden.

Für die Objektblende wurde eine dünne Folie gewählt weil, sich in dickerem Material keine ausreichend kleinen Bohrungen herstellen lassen. Ionen, die die Metallfolie durchqueren, werden dort nicht vollständig gestoppt, sondern verlieren einen Teil ihrer Energie und unterliegen zusätzlich einer Ablenkung um kleine Winkel durch Streuung im Folienmaterial. Im weiteren Strahlverlauf werden die 


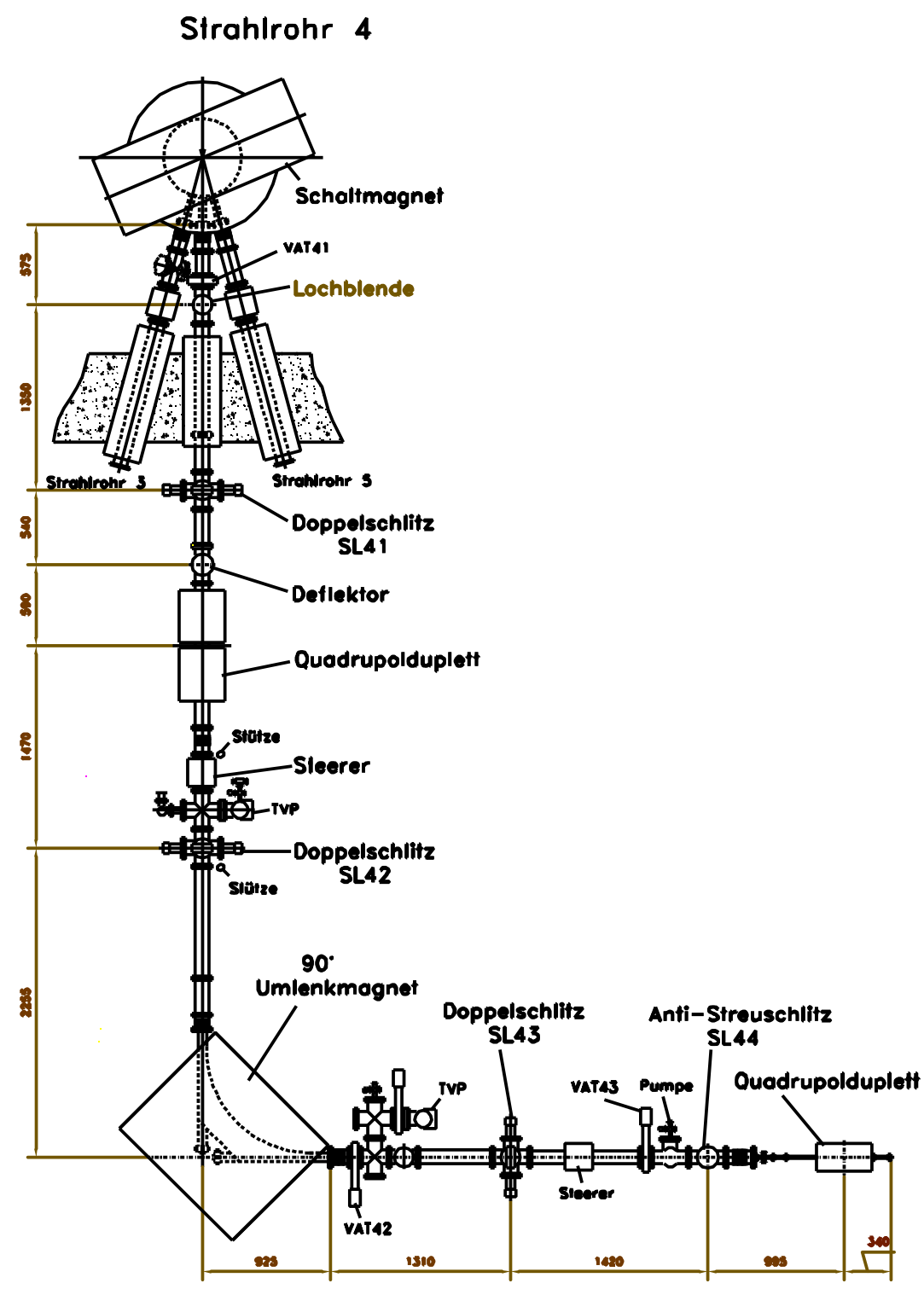

Abbildung 4.1: Plan der Strahlführung. Zur übersichtlicheren Darstellung ist der horizontale Teil in der Aufsicht und der vertikale Teil in der Ansicht dargestellt.

in der Folie gestreuten Ionen durch die energieselektive Abbildung des Analysiersystems aber aussortiert, so dass nur die Ionen, die durch das Loch in der Folie getreten sind, weiter abgebildet werden.

Die in der Folie gestreuten Ionen, im Folgenden als Folienstrahl bezeichnet, können aber auch dazu verwendet werden, die Strahlführung einzustellen. Da die Fläche der Bohrung $(\varnothing=1 \mathrm{~mm}$ ) um einen Faktor 3000 bis 200000 größer ist 


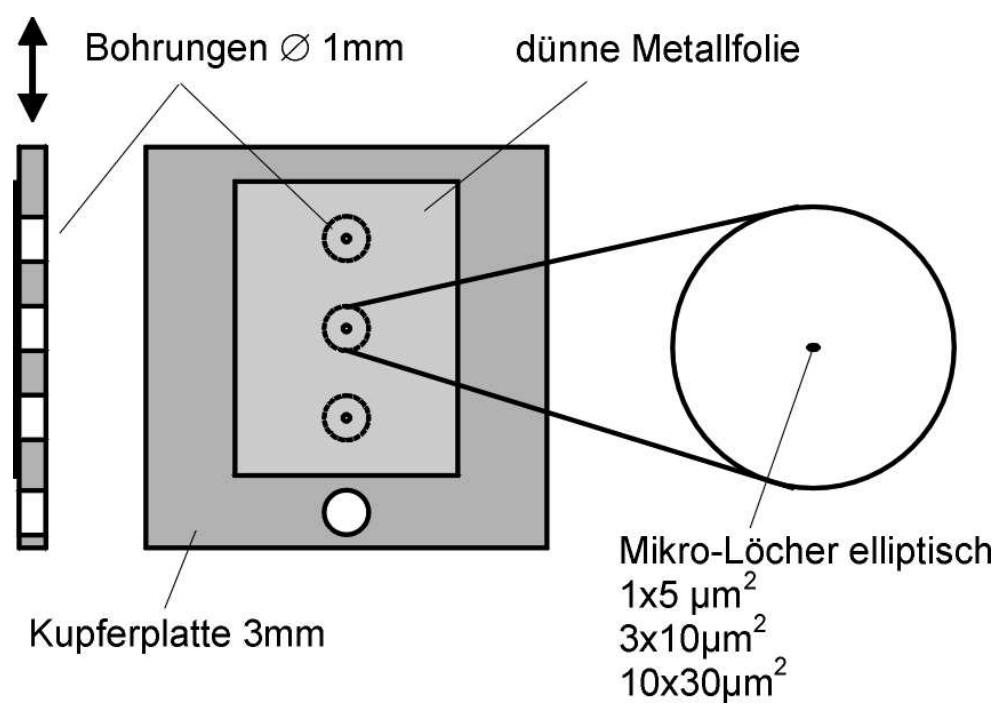

Abbildung 4.2: Objektblende.

als die der Mikrolöcher $(\varnothing=2 \ldots 20 \mu m)$, können die Eigenschaften des Folienstrahls mit den Diagnoseelementen einfacher bestimmt werden. Nach der Einstellung der Magnete mit dem Folienstrahl werden alle Magnetströme um einen kleinen Betrag korrigiert, der der Impulsdifferenz zwischen Folienstrahl und ungestreutem Strahl entspricht.

Die Objektfolie muss so gewählt werden, dass der Energieverlust der gestreuten Ionen ausreicht, um Folienstrahl vom ungestreuten Strahl mit dem Analysiersystem zu trennen. Hierbei ist zu beachten, dass der Folienstrahl im Vergleich zum ungestreuten aufgrund der statistischen Natur der Energieabgabe eine breitere Energieverteilung besitzt. Zu einer vollständigen Trennung sollte die Differenz der Energien zumindest vier Standardabweichungen der Energieverteilung des Folienstrahls betragen. Andererseits soll der Energieverlust und die Winkelaufstreuung des Strahls nicht zu hoch sein, da mit diesem Strahl die Strahlführung eingestellt wird. Eine hohe Winkelaufstreuung hat den Nachteil, dass der Strom je Emittanzvolumen verringert wird.

Die Ablenkung wird durch die Coulomb-Streuung an den Atomkernen verursacht. Für nicht allzu dünne Streuer kann die Winkelverteilung als gaußförmig angenommen werden. Die Standardabweichung dieser Verteilung wird als mittlerer Öffnungswinkel $\Theta_{0}$ angesehen. Zur Berechnung dieses Winkels in mrad kann 
man die Näherungsformel

$$
\Theta_{\circ}=\frac{19.2 \mathrm{MeV}}{p c \beta} Z \sqrt{\frac{L}{L_{\text {rad }}}}\left(1+0.038 \cdot \ln \frac{L}{L_{\text {rad }}}\right)
$$

benutzen [40]. Mit:

$$
\begin{array}{lll}
p & : & \text { Impuls des Teilchens } \\
\beta & : & v / c \\
Z & : & \text { Ladung des Ions } \\
L & : & \text { Dicke des Streuers } \\
L_{\text {rad }} & : & \text { Strahlungslänge des Streumaterials }
\end{array}
$$

In Tabelle 4.1 sind einige Parameter des Folienstrahls dargestellt. Als Objektfolie wird eine $4 \mu \mathrm{m}$ starke Titan-Folie verwendet. Für niedrige Teilchenenergien könnte man auch dünnere Folien verwenden, es ist jedoch schwierig, dünnere Folien mit konstanter Dicke und ohne Poren herzustellen. Es zeigt sich, dass für höherenergetische Protonen ab ca. $10 \mathrm{MeV}$ eine dickere Folie verwendet werden muss. Deshalb wurde eine zweite Objektblende mit einer $5 \mu \mathrm{m}$ starken Molybdän-Folie im Strahlengang platziert, die wahlweise verwendet werden kann.

Im Abstand von 2,05 $m$ hinter der Objektblende befindet sich ein Doppelschlitz (Schlitz 41), der den Öffnungswinkel des Strahls begrenzt. Hiermit kann der Strahlstrom reduziert werden. Zudem wird durch die Verringerung der Divergenz des Strahls der Einfluss der Aberrationen verringert (s. Kapitel 3.2). Wie bei allen eingesetzten Schlitzen, ist jede einzelne Schlitzbacke mittels eines Computerprogramms mit einem Schrittmotor vom Mess- und Bedienungsraum aus fahrbar. Die Einstellgenauigkeit dieses Programms beträgt $1 \mu \mathrm{m}$. Präzise Messungen der Positioniergenauigkeit der Schlitze wurden nicht durchgeführt, Messungen des Strahlstroms legen aber nahe, dass diese besser als $10 \mu m$ ist (s. Abschnitt 6.2.2) und somit in der Praxis vollkommen ausreicht. Für die Schlitze des MikroIonenstrahls wurden Systeme, die seit vielen Jahren in der Beschleunigeranlage im Einsatz sind, von Mitarbeitern des Labors modifiziert.

\subsection{Deflektor}

Unmittelbar hinter dem Divergenzschlitz befindet sich der elektrostatische Deflektor. Aufgabe dieses Bauteils ist es, den Ionenstrahl auszuschalten, sobald die 


\begin{tabular}{|l|r|r|r|r|}
\hline $\begin{array}{l}\text { Energie } \\
/ \mathrm{MeV}\end{array}$ & $\begin{array}{r}\text { Restenergie } \\
\text { / } \mathrm{MeV}\end{array}$ & $\begin{array}{r}\text { Breite } \\
\text { / keV }\end{array}$ & $\begin{array}{r}\text { Differenz } \\
/ \mathrm{keV}\end{array}$ & $\begin{array}{r}\Theta_{0} \\
\text { / mrad }\end{array}$ \\
\hline \hline \multicolumn{3}{|l|}{ Protonen } \\
\hline 3 & 2,87 & 12 & 130 & 22 \\
5 & 4,91 & 12 & 90 & 13 \\
10 & 9,95 & 11 & 50 & 6,7 \\
15 & 14,96 & 11 & 40 & 4,5 \\
20 & 19,97 & 11 & 30 & 3,4 \\
\hline \hline$\alpha$-Teilchen & & & & \\
\hline 3 & 1,58 & 39 & 1420 & 44 \\
5 & 4,02 & 27 & 980 & 27 \\
10 & 9,40 & 23 & 600 & 13 \\
15 & 14,55 & 23 & 450 & 8,9 \\
20 & 19,64 & 23 & 360 & 6,7 \\
\hline
\end{tabular}

Tabelle 4.1: Parameter des Folienstrahls. Der Energieverlust wurde mit dem Programm SRIM berechnet [41].

gewünschte Anzahl ionisierender Teilchen das Target erreicht hat. Der Deflektor besteht aus zwei parallelen Edelstahlplatten, die im Abstand von $8 \mathrm{~mm}$ voneinander isoliert im Vakuumrohr eingebaut sind. Wenn beide Platten auf Massepotential sind, wird der Strahl unbeeinflusst durchgelassen. Wird dagegen zwischen den Platten eine Spannung angelegt, erfährt der Strahl eine Ablenkung um den Winkel:

$$
\phi=\frac{q L}{p v} \frac{U}{d},
$$

mit

$$
\begin{array}{lll}
\phi & : & \text { Ablenkwinkel in Bogenmaß } \\
q & : & \text { Ladung des Ions } \\
L & : & \text { Länge des Deflektors }(=300 \mathrm{~mm}) \\
p & : & \text { Impuls des Ions } \\
v & : & \text { Geschwindigkeit des Ions } \\
U & : & \text { Spannung zwischen den Deflektorplatten }(=600 \mathrm{~V}) \\
d & : & \text { Plattenabstand. }(=8 \mathrm{~mm})
\end{array}
$$


Die so erreichte Winkeländerung bewirkt durch die nachfolgende ionenoptische Abbildung eine Abweichung des Strahls von der Sollposition. Bei einer geeigneten Wahl der Schlitzweite des Energieeingangsschlitzes (Schlitz 42) kann der Strahl vollständig „ausgeschaltet” werden. Für $20 \mathrm{MeV}$ Protonenergibt sich ein Ablenkwinkel von ca. 0,2 mrad. Daraus resultiert an Schlitz 42 eine Ortsabweichung von ca. $0,5 \mathrm{~mm}$.

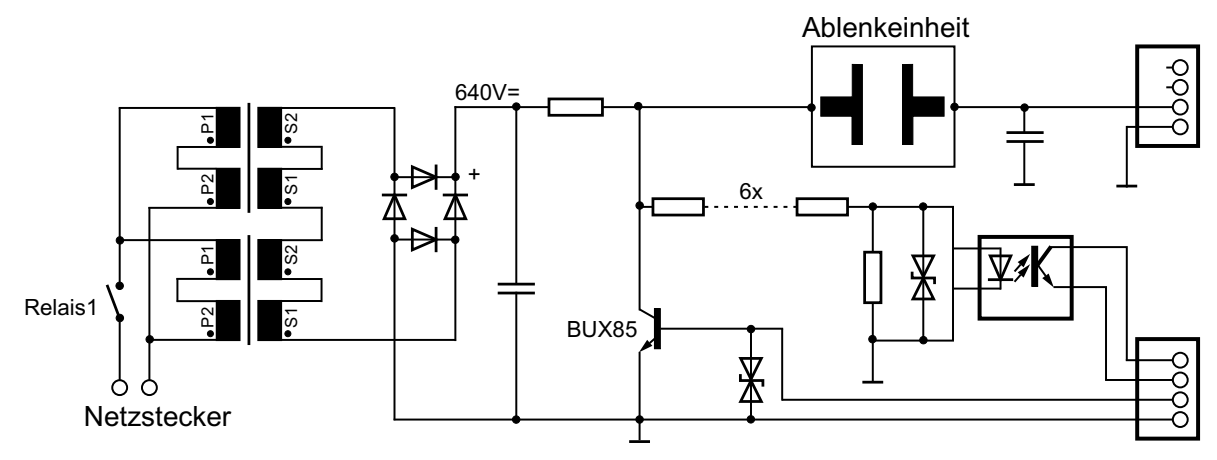

Abbildung 4.3: Schaltplan der Leistungsstufe des elektrostatischen Deflektors.

Die Geschwindigkeit, mit der der Deflektor von Durchgang auf Sperrung schalten muss, hängt von der Teilchenrate des Strahls und von der tolerierbaren Fehlerquote ab. Um bei einer angenommenen Teilchenrate von $1000 s^{-1}$ eine Fehlerquote von $1 \%$ zu erreichen, ist eine Schaltzeit von ca. $10 \mu s$ notwendig. Der Schaltplan der Deflektorelektonik ist in Abbildung 4.3 gegeben. Wichtigstes Bauteil ist der Hochspannungstransistor (BUX85), der die gleichgerichtete Spannung in kurzer Zeit schalten muss. Die Verwendung dieses Transistors begrenzt die verwendbare Spannung auf maximal 800 V. Bei höheren Werten müssten Röhren verwendet werden.

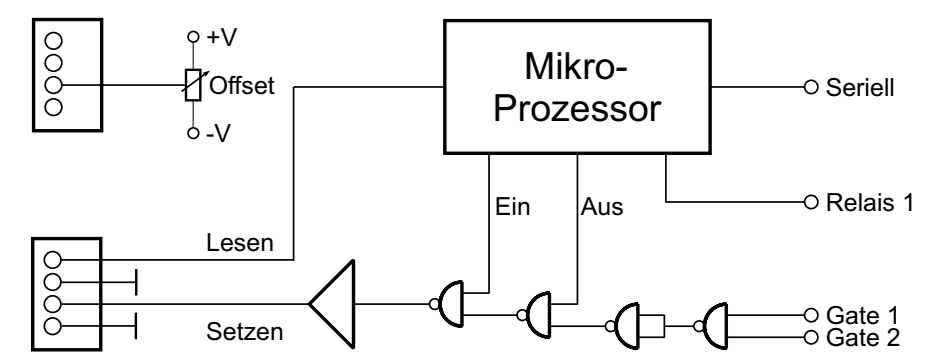

Abbildung 4.4: Schaltplan der Ansteuerung des Deflektors. 
Die Schaltung zur Ansteuerung der Leistungsstufe ist in Abbildung 4.4 dargestellt. Die Ansteuerung des Deflektors geschieht auf zwei Arten. Mittels zweier Gate-Eingänge kann der Deflektor schnell ein- und ausgeschaltet werden. Außerdem kann der Deflektor über ein serielles Signal dauerhaft auf Sperrung oder Durchlass gestellt werden. Das schnelle Ausschalten des Strahls wird mit einem TTL-Signal aus dem Zählwerk der Detektorelektronik realisiert. Wenn die voreingestellte Teilchenzahl erreicht ist (Preset), wechselt der Intervall-Ausgang des Zählers innerhalb von 100 ns von high auf low und schaltet den Deflektor. Die Durchschaltung des Strahls erfolgt, wenn der Intervall-Ausgang des Zählers wieder auf high zurückschaltet. Dies geschieht, nachdem die Ablaufsteuerung (s. Kapitel 5.5) die nächste Bestrahlungsposition angefahren und den Zähler mittels eines seriellen Signals wieder auf aktiv gesetzt hat. Die technische Realisierung des Deflektors und seiner Ansteuerelektronik wurde von Mitarbeitern des Fachlabors übernommen.

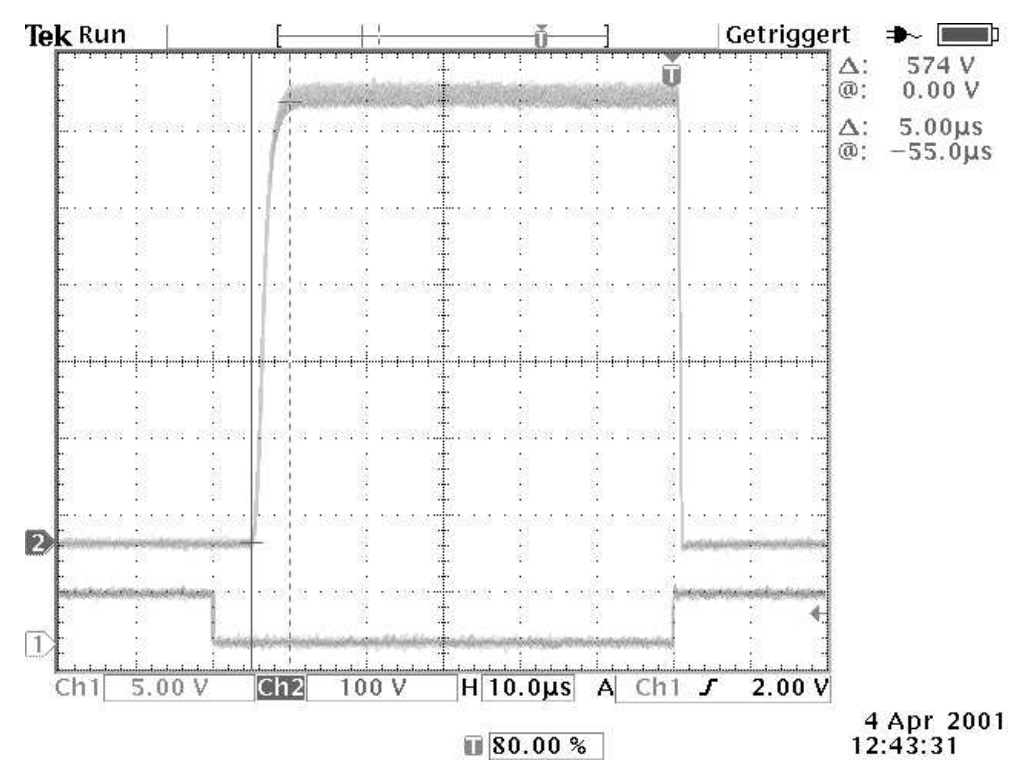

Abbildung 4.5: Zeitlicher Verlauf der Ablenkspannung am Deflektor (Signal 2) und Verlauf der Ansteuerspannung (Signal 1). Der Abstand der horizontalen Gitternetzlinien beträgt $10 \mu s$.

In Abbildung 4.5 sind die Anstiegs- und Abstiegsflanke der Deflektorspannung gezeigt. Die untere Spannung ist der Verlauf des Steuersignals, das den Anstieg und Abfall auslöst. Die Zeitverzögerung des Deflektorsignals beträgt ca. $5 \mu s$ und die Anstiegsflanke ebenfalls ca. $5 \mu s$. 
Die Zeitverzögerung beim Wiedereinschalten des Strahls beträgt nur $1 \mu s$. Diese ist allerdings nicht kritisch. Wichtiger ist die Konstanz des Massepotentials während der Bestrahlung. Messungen ergeben hier eine Abweichung von maximal $50 m V p-p$. Der Einfluss geringer Spannungen am Deflektor ist mithilfe der Offsetverstellung untersucht worden. Wie experimentell geprüft wurde, bewirkt eine Verstellung von $+5 \mathrm{~V}$ nach $-5 \mathrm{~V}$ eine axiale Verschiebung eines Protonenstrahls von $3 \mathrm{MeV}$ um weniger als $2 \mu \mathrm{m}$. Höherenergetische Teilchen sind unempfindlicher, so dass ein störender Einfluss des Deflektors durch Schwankungen der Nullspannung im Durchlass ausgeschlossen werden kann.

\subsection{Magnete}

In Kapitel 3.4 wurden die Ergebnisse einiger Strahlführungsrechnungen präsentiert. Den Rechnungen liegen die tatsächlichen technischen Spezifikationen der verwendeten Magnete zugrunde.

\subsubsection{Dipolmagnet}

\begin{tabular}{|l|r|}
\hline Maximales Feld & $1,02 \mathrm{~T}$ \\
Ablenkradius & $0,75 \mathrm{~m}$ \\
Ablenkwinkel & $90^{\circ}$ \\
Polschuhabstand & $32 \mathrm{~mm}$ \\
Polschuhbreite & $112 \mathrm{~mm}$ \\
Eingangs- \& Ausgangswinkel & $27^{\circ}$ \\
Polschuhform & Approx. Rogowsky \\
\hline Homogenität & $10^{-3}( \pm 20 \mathrm{~mm})$ \\
Windungszahl & 90 \\
Maximaler Strom & $158 \mathrm{~A}$ \\
DAC & $16 \mathrm{bit}$ \\
Stabilität & $1700 \mathrm{~kg}$ \\
Gewicht & $3 \mathrm{ppm}(30 \mathrm{~min})$ \\
\hline
\end{tabular}

Tabelle 4.2: Parameter des Dipols.

Die wichtigen Parameter des Dipolmagneten sind in Tabelle 4.2 aufgelistet. Durch die Kantenwinkel hat er energieselektive Abbildungseigenschaften wie in Abschnitt 2.8.3 beschrieben. Das Feld des Dipols wird während der Experimente mit 
einer NMR-Sonde mit hoher Präzision $( \pm 10 m G)$ gemessen. Durch diese Messung können auch die Impulsdifferenz zwischen Folienstrahl und Mikrostrahl bestimmt und die Quadrupolströme entsprechend skaliert werden.

\subsubsection{Quadrupolmagnete}

In erster Näherung ist die Brennweite eines Quadrupols gegeben durch

$$
f=\frac{1}{\sqrt{k} \sin (\sqrt{k} L)}, \quad \text { mit } \quad k=\frac{q g}{p_{0}}=\frac{1}{(B \rho)_{0}},
$$

mit

$$
\begin{array}{lll}
L & : & \text { Länge des Magneten } \\
(B \rho)_{0}: & \text { Magnetische Steifigkeit des Ions. }
\end{array}
$$

\begin{tabular}{|l|r|r|}
\hline & 1. Dublett & 2. Dublett \\
\hline Maximales Feld /T & 0,5 & 0,55 \\
Effektive Länge /mm & 285 & 112 \\
Halbapertur /mm & 50 & 10 \\
\hline
\end{tabular}

Tabelle 4.3: Parameter der Quadrupolmagnete

Der maximale Gradient eines Quadrupolmagneten lässt sich in der Regel nur durch eine Verringerung der Apertur erhöhen, da das maximale Feld ohne Supraleitung auf ca. 0,5 Tesla begrenzt ist. Neben der Apertur bleibt als weiterer Parameter die Länge des Magneten, um eine gewünschte Brennweite zu erreichen:

$$
L=\frac{1}{\sqrt{k}} \arcsin \left(\frac{1}{\sqrt{k} f}\right) .
$$

Aus den Strahlführungsrechnungen ergibt sich, dass die mikrofokussierenden Quadrupole eine Brennweite von ca. $15 \mathrm{~cm}$ erreichen müssen. Bei einem Gradienten von $55 \mathrm{~T} / \mathrm{m}$ ergibt sich hieraus eine Magnetlänge von ca. $12 \mathrm{~cm}$. Um den hohen Gradienten zu erreichen, wurden Magnete mit einer Halbapertur, das ist die halbe räumliche Differenz zwischen gegenüberliegenden Polschuhen, von $1 \mathrm{~cm}$ konstruiert. 


\subsection{Schlitzstrommessung}

Die Messung des auf den Schlitzen auftreffenden Strahlstromes ist eine einfache aber wichtige Methode, die Strahllage zu bestimmen. Für den Mikro-Ionenstrahl wurde im Rahmen dieser Arbeit ein Strommessystem entwickelt, dass die präzise Messung von insgesamt 16 Schlitzströmen in einem Messbereich zwischen $1 p A$ und $500 \mathrm{nA}$ bewerkstelligt. Der symbolische Schaltplan der Messgeräte ist in Abbildung 4.6 dargestellt. Wesentliches Element der Schaltung ist ein rauscharmer, schaltbarer Integrator (Typ ACF2101 [42]). Durch den Eingangsschalter kann die Integrationszeit beliebig lang gewählt werden, ohne dass der Eingangsruhestrom des Bauteils ständig aufintegriert wird. Hierdurch wird es moglich, Integrationszeiten von mehreren Sekunden einzustellen und Ströme im Bereich unterhalb von $1 p A$ zu messen.

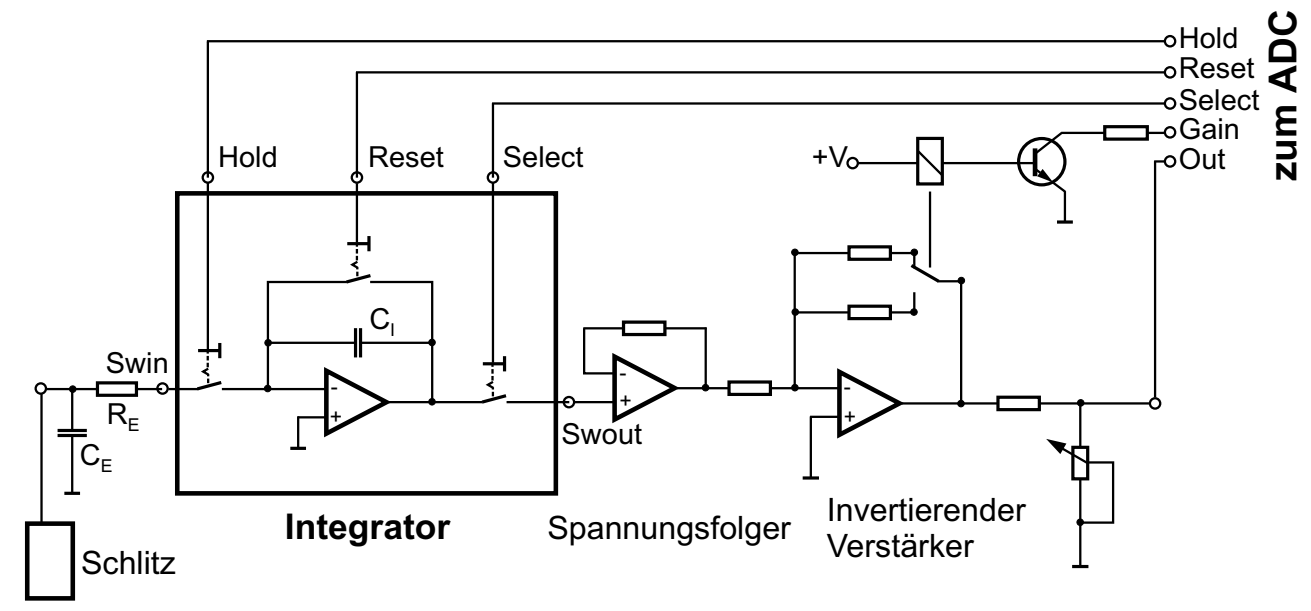

Abbildung 4.6: Schaltplan der Strommesselekronik.

In Tabelle 4.4 sind Kalibriermessungen der Kanäle eins bis acht in vier verschiedenen Messbereichen zusammengestellt. Sie wurden mit einer Stromquelle unter Laborbedingungen durchgeführt. Die Übereinstimmung der verschiedenen Kanäle untereinander und mit dem Sollwert ist für die Justierung des Strahls vollkommen ausreichend. Eine individuelle Korrektur jedes Kanals wurde nicht vorgenommen, könnte aber bei Bedarf leicht durchgeführt werden. In der Experimentierhalle ergibt sich eine zusätzliche Unsicherheit des gemessenen Stroms von ca. $2 p A$, da dort ein Störsignal auftritt, das eine Schwebung des Integratorsignals verursacht. Dieser Effekt beschränkt die Zuverlässigkeit der Strommessung zur Zeit auf den Bereich oberhalb von $10 p A$. 


\begin{tabular}{|c|c|c|c|c|c|c|c|c|}
\hline Messbereich & \multicolumn{2}{|c|}{$5 \mathrm{nA}$} & \multicolumn{2}{|c|}{$2,5 \mathrm{nA}$} & \multicolumn{2}{|c|}{$150 \mathrm{pA}$} & \multicolumn{2}{|c|}{$15 \mathrm{pA}$} \\
\hline$T_{\text {int }}$ & \multicolumn{2}{|c|}{$60 \mathrm{~ms}$} & \multicolumn{2}{|c|}{$600 \mathrm{~ms}$} & \multicolumn{2}{|c|}{$300 \mathrm{~ms}$} & \multicolumn{2}{|c|}{$3000 \mathrm{~ms}$} \\
\hline Verstärkung & \multicolumn{2}{|c|}{1} & \multicolumn{2}{|c|}{1} & \multicolumn{2}{|c|}{33} & \multicolumn{2}{|c|}{33} \\
\hline Kanal \# & $\begin{array}{c}\text { Steigung } \\
\text { /bit/nA }\end{array}$ & $\begin{array}{c}\text { Offset } \\
/ \mathrm{nA}\end{array}$ & $\begin{array}{l}\text { Steigung } \\
\text { /bit/nA }\end{array}$ & $\begin{array}{c}\text { Offset } \\
/ \mathrm{nA}\end{array}$ & $\begin{array}{l}\text { Steigung } \\
\text { /bit/pA }\end{array}$ & $\begin{array}{c}\text { Offset } \\
/ \mathrm{pA}\end{array}$ & $\begin{array}{l}\text { Steigung } \\
\text { /bit/pA }\end{array}$ & $\begin{array}{c}\text { Offset } \\
/ \mathrm{pA}\end{array}$ \\
\hline 1 & 40,73 & 0,02 & 405,9 & 0,007 & 6,746 & $-4,2$ & 67,66 & $-0,43$ \\
\hline 2 & 40,62 & 0,03 & 405,9 & 0,006 & 6,743 & $-5,2$ & 67,53 & $-0,51$ \\
\hline 3 & 2,28 & 0,03 & 422,6 & 0,005 & 7,060 & $-4,7$ & 70,63 & $-0,46$ \\
\hline 4 & 42,31 & 0,01 & 422,4 & 0,006 & 7,076 & -11 & 70,62 & $-1,10$ \\
\hline 5 & 40,88 & 0,03 & 409,4 & 0,006 & 6,795 & $-3,7$ & 68,02 & $-0,30$ \\
\hline 6 & 40,98 & 0,03 & 409,7 & 0,004 & 6,815 & $-4,7$ & 68,15 & $-0,43$ \\
\hline 7 & 40,77 & 0,05 & 409,0 & 0,007 & 6,794 & $-3,4$ & 67,87 & $-0,29$ \\
\hline 8 & 40,98 & 0,03 & 409,1 & 0,007 & 6,803 & $-4,7$ & 68,07 & $-0,44$ \\
\hline Mittelwert & 41,19 & & 411,7 & & 6,854 & & 68,57 & \\
\hline$\sigma_{r e l}$ & $1,6 \%$ & & $1,5 \%$ & & $1,8 \%$ & & $1,8 \%$ & \\
\hline
\end{tabular}

Tabelle 4.4: Verstärkungsfaktoren und Offsetspannungen der Schlitzstrom-Messgeräte.

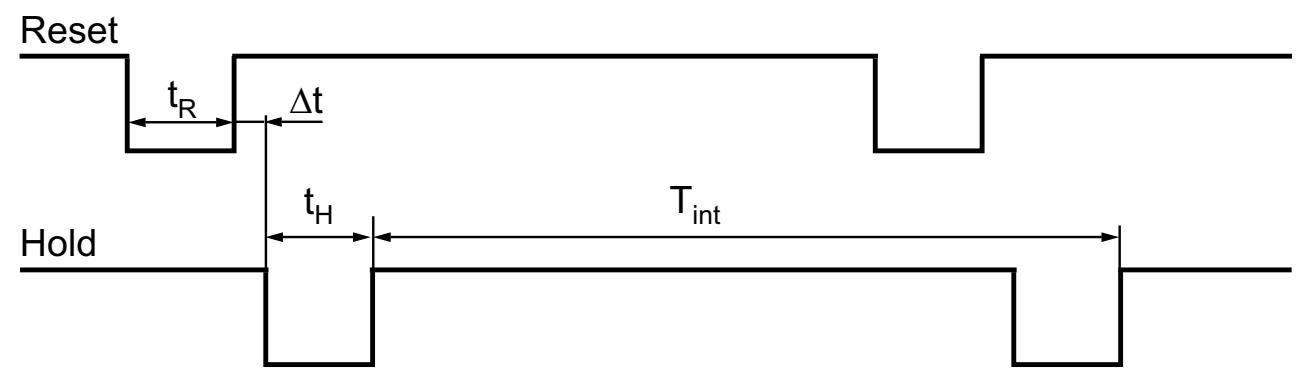

Select 1

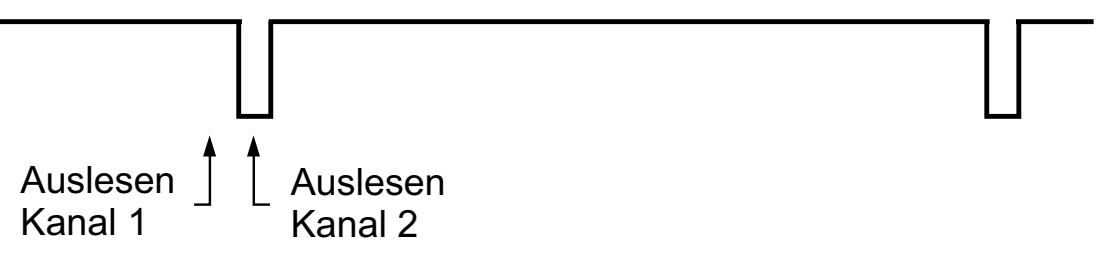

Abbildung 4.7: Zeitlicher Verlauf der verschiedenen Steuersignale der SchlitzstromMessgeräte.

Der Integrator benötigt eine Sequenz von Steuersignalen zum Starten, Auslesen, Stoppen und Zurücksetzen der Messung. Diese sind in Abbildung 4.7 dargestellt. Wichtigster Parameter ist die Integrationszeit $T_{i n t}$, durch welche die Länge eines 
Messzyklus gegeben ist. Die Zeitdauer $t_{R}$ bezeichnet die Schließung des ResetSchalters, der das Zurücksetzen des Messwertes auf null vor Beginn der nächsten Messung bewirkt. Die Zeitdauer $t_{H}$ bezeichnet die Schließung des Hold-Schalters. Dieses bewirkt das Abfließen der auf einem Schlitz angesammelten Ladung. Die beiden Zeiten $t_{R}$ und $t_{H}$ können nahezu beliebig gewählt werden, solange die Zeitkonstanten für das Auf- und Entladen des Integrationskondensators berücksichtigt werden. Beide wurden auf $20 \mathrm{~ms}$ gesetzt, da das einer vollen Periode der Netzfrequenz entspricht, so dass das integrierte Störsignal dieser Frequenz demzufolge verschwindet. Die Auslese zweier Messwerte durch einen ADC wird durch das Select-Signal erreicht.

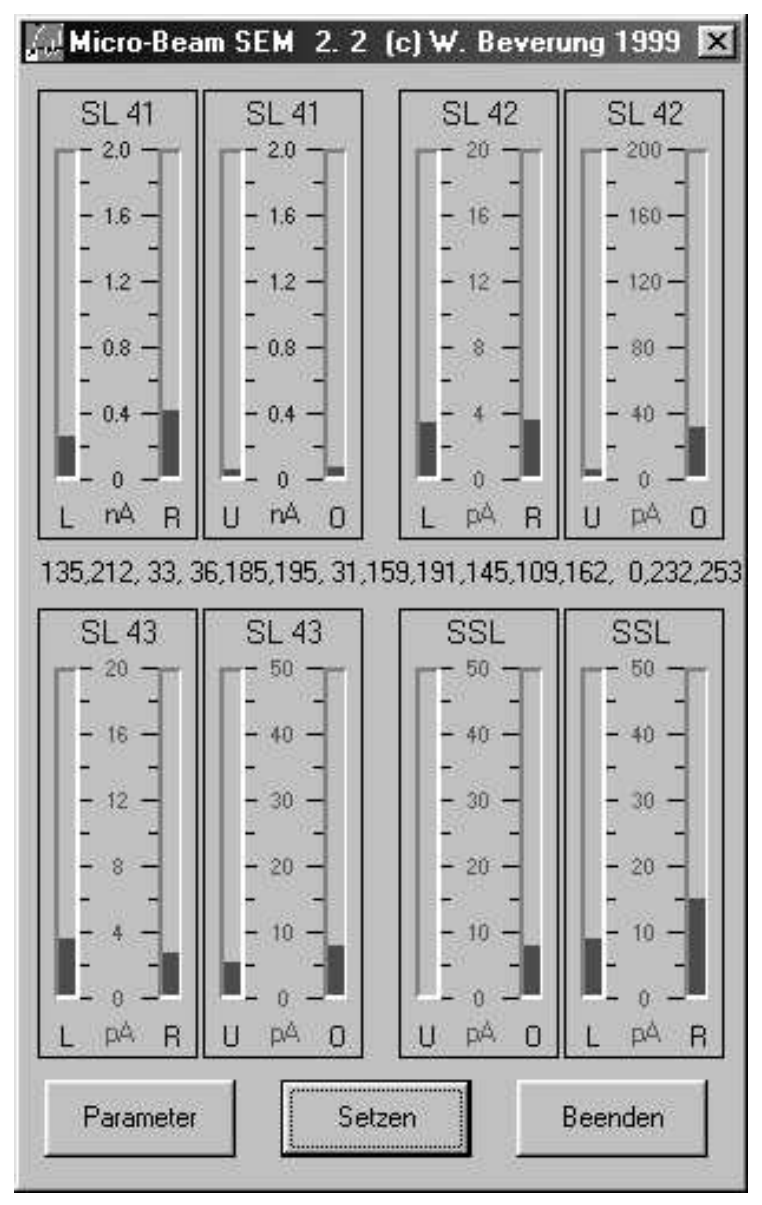

\begin{abstract}
Abbildung 4.8: Darstellung der Schlitzströme am Messrechner. Die Ausgabewerte des ADC sind als Balkengrafik dargestellt und zur Sicherheit sowie zur genaueren Quantifizierung zusätztlich als Zahlen aufgelistet. Der Messbereich der einzelnen Kanäle kann menügeführt variiert werden.
\end{abstract}

Die analogen Messwerte werden über ein Bussystem an eine Steuerplatine übertragen. Dort werden sie digitalisiert und seriell an einen Messrechner übertragen, der sie graphisch darstellt (s. Abb. 4.8). Das Programm erlaubt auch die Variation von Messparametern wie z.B. Integrationszeit und Verstärkungsfaktor. Die Signalübertragung und Darstellung der analogen Messwerte auf einem Computer wurde von Mitarbeitern des Labors realisiert. 


\section{Kapitel 5}

\section{Experimentierplatz}

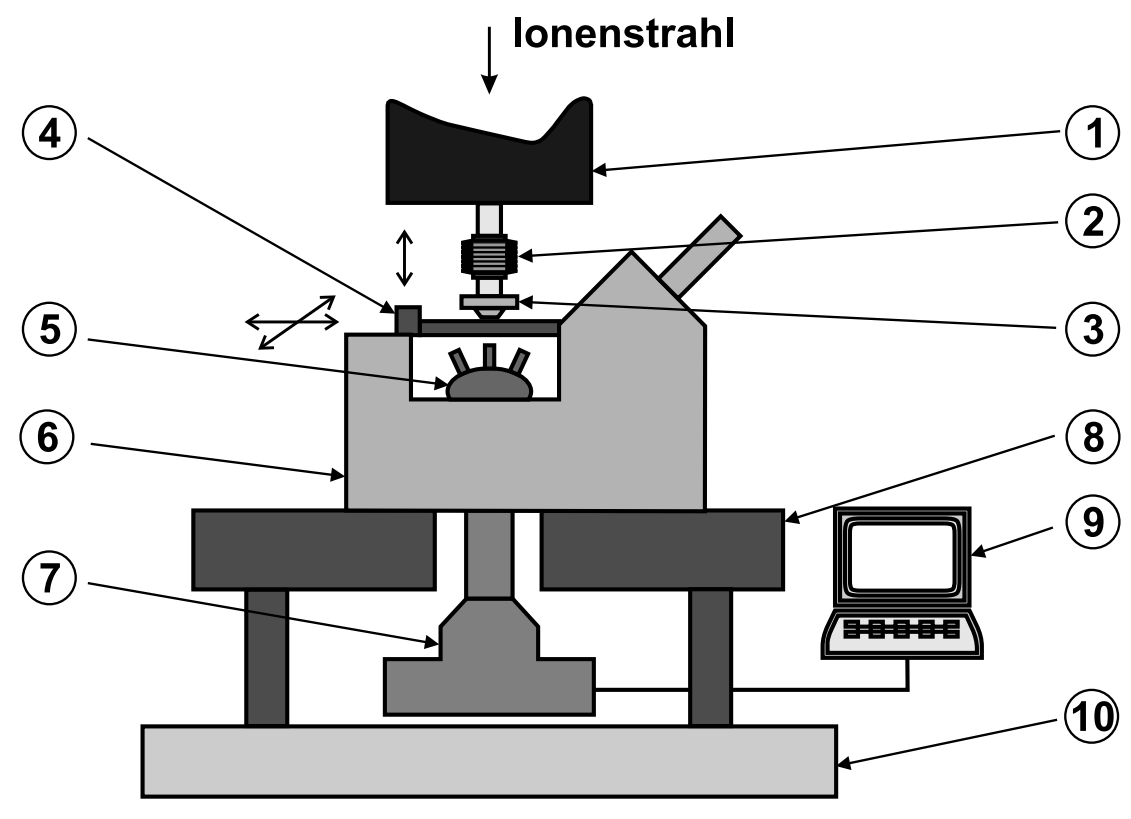

Abbildung 5.1: Schematische Darstellung des Experimentierplatzes.

Der Aufbau des Experimentierplatzes ist in Abbildung 5.1 schematisch dargestellt. Er besteht aus folgenden Komponenten:

1 : Mikrofokussierendes Quadrupoldublett

2 : Federbalg mit Hebemechanik für das Vakuumfenster

3 : Strahlaustrittsfenster

4 : Mikropositioniertisch (XY-Stage)

5 : Objektivrevolver mit Objektiven und Detektoren

6 : Inverses Mikroskop

7 : Slow-Scan CCD-Kamera

8 : Vibrationsarmer optischer Tisch

9 : Computer

10: Betonfußboden 


\subsection{Strahldiagnose mit Szintillator und CCD-Kamera}

Wichtigstes Element zur Lokalisierung des Ionenstrahls und zur Einstellung der optimalen Strahleigenschaften sind ein Szintillator und eine CCD-Kamera. Der Szintillator befindet sich hierbei unmittelbar hinter dem Strahlaustrittsfenster. Durch die zweidimensionale Abbildung entsteht ein intuitiver Eindruck der Strahlparameter Position, Breite und Form.

Der Szintillator zur Strahldiagnose muss folgende Bedingungen erfüllen:

- Hohe Lichtausbeute für Ionen

- Wellenlänge der Emission angepasst an die Quanteneffizienz der Kamera

- Leichte Handhabung, Robustheit

Das Material YAG:Ceำ wird in der Elektronenmikroskopie routinemäßig verdendet und ist in vielen verschiedenen Formen kommerziell erhältlich. Gegenüber den Plastikszintillatoren, welche bei der Einzelteilchendetektion zum Einsatz kommen (s. Kapitel 5.2), hat es den Vorteil der größeren Dichte und der Lichtemission mit einer größeren Wellenlänge. Das Material ist glasartig und robust in der Handhabung und kann unter normalen Umgebungsbedingungen gelagert werden. Mit langen Integrationszeiten $(\geq 60 s)$ konnten sehr geringe Strahlströme von ca. 100 $\alpha$-Teilchen pro Sekunde visualisiert werden.

\begin{tabular}{|l|r|}
\hline Dichte & $4,55 \mathrm{~g} / \mathrm{cm}^{3}$ \\
Wellenlänge der Emission & $550 \mathrm{~nm}$ \\
Photonenausbeute für Elektronen & $c a .20000 \mathrm{MeV}^{-1}$ \\
\hline
\end{tabular}

Tabelle 5.1: Parameter des YAG:Ce Szintillators [43].

Zur Darstellung des Szintillationslichtes bei geringem Ionenstrom ist eine hohe Empfindlichkeit der Kamera notwendig. Des Weiteren sind eine hohe Auflösung, also eine große Pixelanzahl und kleine Pixelabmessungen erforderlich, da die Vergrößerung des Objektivs wegen des großen Arbeitsabstandes in den meisten Fällen auf $\times 20$ beschränkt ist. In Tabelle 5.2 sind die wesentlichen Spezifikationen der CCD-Kamera aufgelistet.

\footnotetext{
${ }^{1}$ Mit Cer dotiertes Yttrium-Aluminium-Garnet $Y_{2} \mathrm{Al}_{5} \mathrm{O}_{10}$.
} 


\begin{tabular}{|l|r|}
\hline CCD-Chip & Kodak $1401 \mathrm{E}$ \\
Anzahl der Pixel & $1315 \times 1033$ \\
Pixelabmessungen & $6,8 \mu \mathrm{m} \times 6,8 \mu \mathrm{m}$ \\
Integrationszeit & $80 \mu \mathrm{s}$ bis $17 \mathrm{~min}$ \\
Quanteneffizienz bei $550 \mathrm{~nm}$ & $\approx 55 \%$ \\
Kühlung (Peltier) $^{a}$ & $-12^{\circ} \mathrm{C}$ \\
Binning Modus $^{a}$ & $1 \times 1,2 \times 2,3 \times 3,4 \times 4$ \\
\hline
\end{tabular}

${ }^{a}$ Binning Modus bezeichnet die analoge Addition der Ladung benachbarter Pixel.

Tabelle 5.2: Parameter der Slow-Scan CCD-Kamera [44].

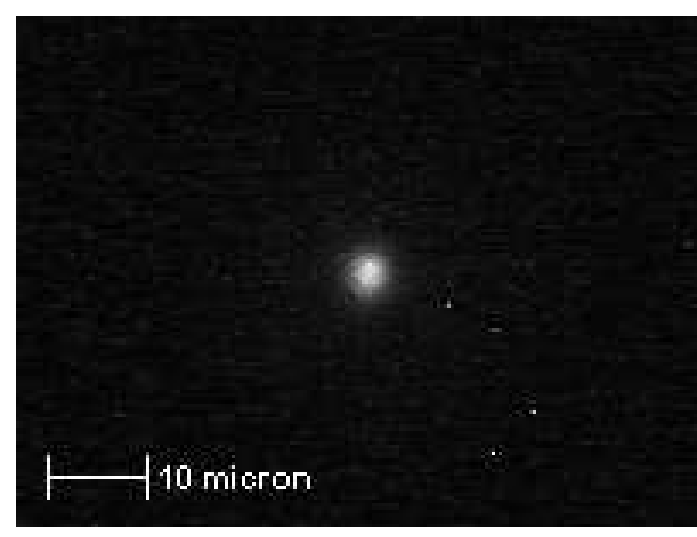

Abbildung 5.2: Strahlfleck bei optimalen Abbildungseigenschaften.

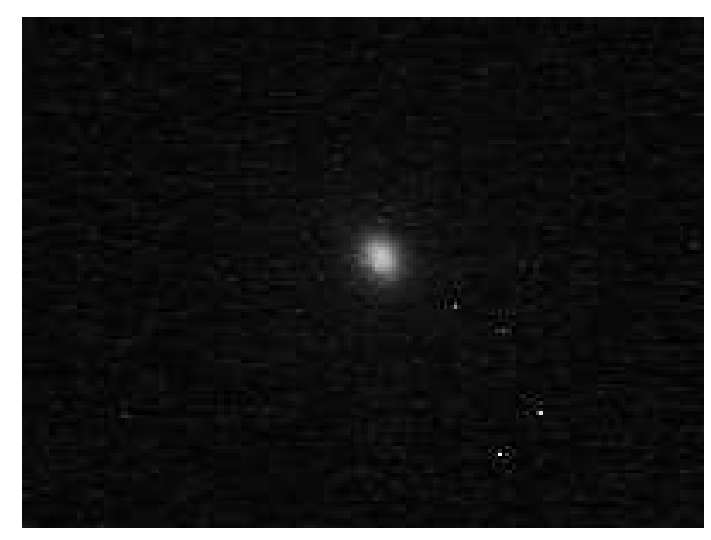

Abbildung 5.3: Strahlfleck bei leicht geänderten Magnetwerten.

Die Abbildungen 5.2 und 5.3 verdeutlichen die Sensitivität des visuellen Eindrucks. Zwischen beiden Bildern wurde der Strom von nur einem der vier Quadrupolmagnete um ca. 1\% geändert. Am Computerbildschirm sind die veränderten Strahlparameter am Fokuspunkt auch ohne eine Quantifizierung der Bilder leicht zu erkennen. Dies ermöglicht eine unkomplizierte Optimierung der Abbildungseigenschaften.

\subsubsection{Einfluss der Schärfentiefe}

Mit einem optischen Mikroskop wird ein Objekt scharf abgebildet, wenn es sich genau in der Gegenstandsweite vor dem Objektiv befindet. Je nach Apertur des Objektivs und Wellenlänge des Lichtes beträgt die Toleranz der Objektposition (Schärfentiefe) nur wenige Mikrometer. Objekte, die eine größere Distanz zum 
Gegenstandspunkt haben, werden unscharf und vergrößert abgebildet. Der Ionenstrahl im Szintillator stellt eine linienförmige Lichtquelle dar, weil Szintillationslicht bis zur maximalen Eindringtiefe produziert wird (Dicke der YAG-Schicht: $50 \mu \mathrm{m}$ ). Daher erscheint er im Mikroskop zwangsläufig größer als er tatsächlich ist. Abbildung 5.4 macht diesen Effekt deutlich.

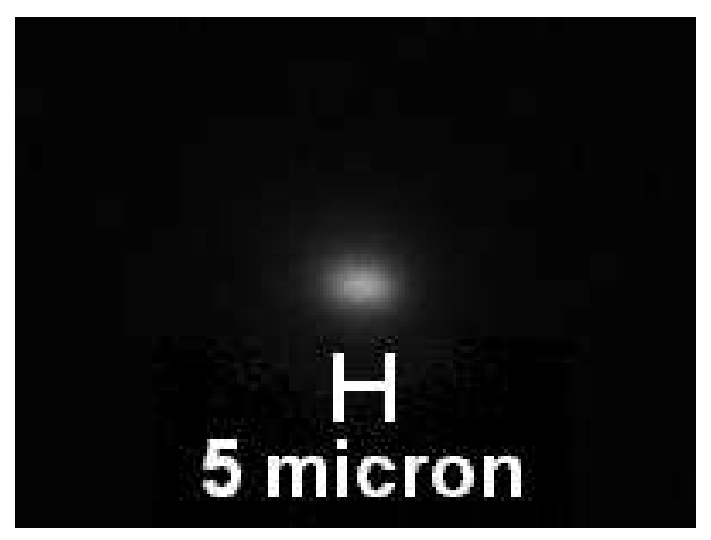

Abbildung 5.4: Strahlfleck bei optimaler Einstellung der Kameraparameter.

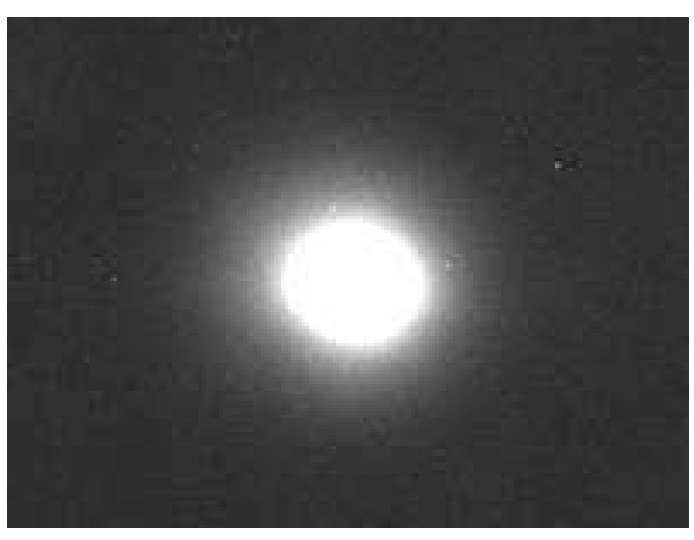

Abbildung 5.5: Strahlfleck bei zu empfindlicher Kameraeinstellung.

Beide Bilder sind bei identischem Ionenstrahl aufgenommen, jedoch mit unterschiedlicher Integrationszeit der Kamera. Bei zu empfindlicher Kamera reicht das defokussiert abgebildete Licht entlang des Ionenstrahls aus, um den Strahlfleck deutlich größer darzustellen. Selbst bei optimalen Kameraparametern ist zu erwarten, dass der Ionenstrahl im Kamerabild immer zu groß erscheint. Der Vergleich mit Messungen mit einem CR39-Spurdetektor (s. Abschnitt 6.2.1) zeigt, dass die Kamerabilder keine genaue Bestimmung der Strahlbreite zulassen. Sie stellen aber im Bereich bis hinunter zu einem Mikrometer ein verlässliches Mittel zur Minimierung der Strahlbreite dar.

\subsection{Detektion einzelner Ionen}

Bei Experimenten mit abgezählten Ionen ist es notwendig, jedes einzelne Ion mit einer Sicherheit nahe $100 \%$ nachzuweisen. Wenn die Energie der Ionen ausreicht, um die Probe vollständig zu durchdringen, können sie leicht mit einem Halbleiterdetektor nachgewiesen werden. Wo dies nicht der Fall ist, wird es notwendig, einen Transmissionsdetektor zu verwenden, der sich vor der Probe befindet.

Das Verfahren, das am PTB Mikro-Ionenstrahl zum Einsatz kommt, wird seit vielen Jahren im Routinebetrieb angewendet [25]. Eine dünne Szintillatorfolie 
wird vor der Probe angebracht und erzeugt einen Lichtblitz bei jedem Durchgang eines Ions. Hinter der Probe ist ein Photonendetektor angebracht (Photo Multiplier Tube, PMT), der das erzeugte Licht in ein elektrisches Signal umwandelt (s. Abb. 5.6). Das Verfahren setzt die Durchsichtigkeit der Probe voraus.

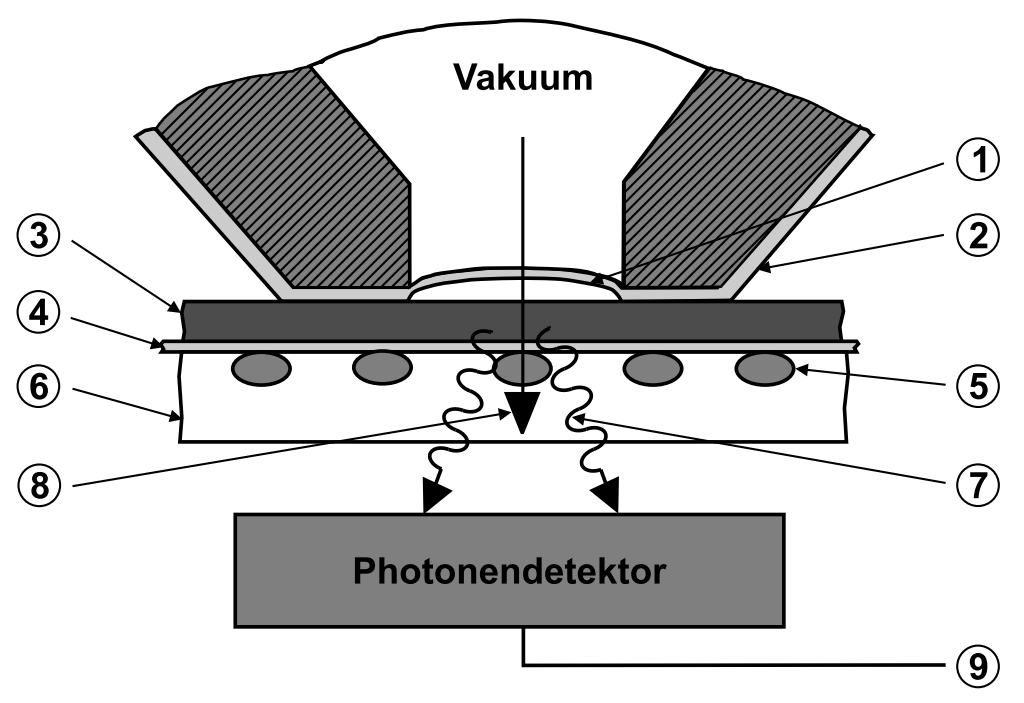

Abbildung 5.6: Schematische Darstellung des Vakuumfensters und der Einzelteilchendetektion.

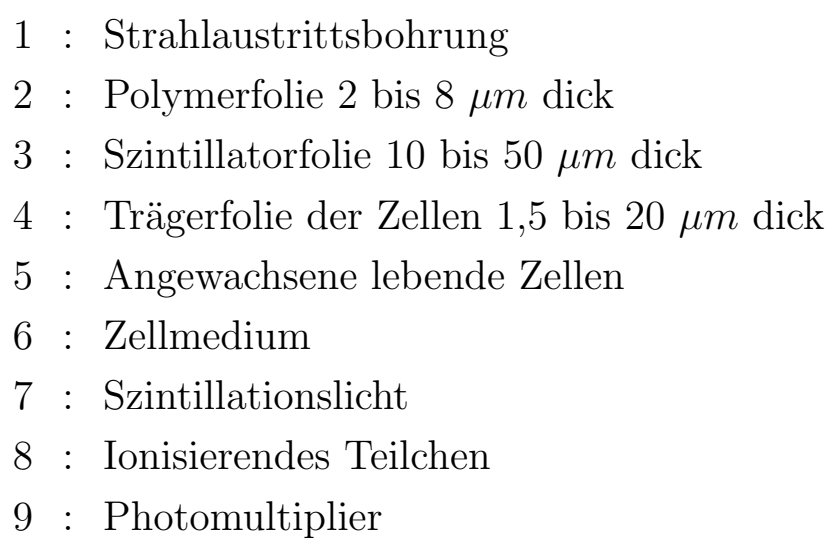

Die Szintillatorfolie verursacht jedoch eine Störung des Ionenstrahls durch Kleinwinkelstreuung (vgl. Abschnitt 5.3). Es muss ein Kompromiss gefunden werden, um bei einer hohen Nachweiswahrscheinlichkeit eine möglichst geringe Störung des Strahls zu erreichen. Die Dicke des Szintillators ist deshalb ein wichtiger Parameter. Am PTB Mikro-Ionenstrahl wird der Plastik-Szintillator BC-400² verwen-

\footnotetext{
${ }^{2}$ Bicron, Newbury (Ohio), USA.
} 
det, der als dünne Folie $(\geq 10 \mu m)$ erhältlich ist. Die Wellenlänge der maximalen Lichtemission für BC-400 beträgt $423 \mathrm{~nm}$.

\begin{tabular}{|l|c|}
\hline Photoelektronenausbeute bei $420 \mathrm{~nm}$ & $\approx 0,25 e^{-} /$Photon \\
Elektronenverstärkung & $1 \times 10^{6}$ \\
Dunkelrate (typ.) & 80Photonen/ Sekunde \\
Betriebsspannung & $800 \mathrm{~V}$ \\
Elektronen-Durchgangszeit & $5,4 \mathrm{~ns}$ \\
\hline
\end{tabular}

Tabelle 5.3: Eigenschaften des R7400P Photomultipliers [45, 46].

Der Photomultiplier muss aufgrund der räumlichen Einschränkungen am Experimentierplatz recht klein sein. Zudem muss er eine hohe Photoelektronenausbeute bei $420 \mathrm{~nm}$ haben und eine geringe Dunkelrate sowie ein geringes Rauschen aufweisen. Die wesentlichen Eigenschaften des Photomultipliers (Type R7400P3) sind in Tabelle 5.3 aufgelistet.

Im Folgenden soll abgeschätzt werden, wieviele Szintillationsphotonen zum sicheren Teilchennachweis benötigt werden: Ein Teilchennachweis liegt dann vor, wenn ein Signal deutlich über dem Signal von zufällig auftreffenden einzelnen Photonen liegt. Dies kann man als gegeben ansehen, wenn in der Photokathode wenigstens drei Photoelektronen gleichzeitig emittiert werden. Da die Emission von Photoelektronen ein statistischer Prozess ist, muss die mittlere Anzahl der Photoelektronen deutlich höher sein. Bei einer mittleren Photoelektronenausbeute von $20 \%$ in der Photokathode benötigt man aufgrund der Binomialverteilung ca. 40 Photonen am Detektor, damit in $99 \%$ der Fälle drei oder mehr Photoelektronen emittiert werden. Da der Photomultiplier nicht den ganzen Raumwinkel um den Szintillator erfasst, wird er nur von einem kleinen Teil des im Szintillator erzeugten Lichtes getroffen wird. Aus dem Durchmesser des PMT von $11 \mathrm{~mm}$ und dem Abstand zum Szintillator von $11 \mathrm{~mm}$ bis $12 \mathrm{~mm}$ berechnet sich, dass ca. $5 \%$ bis $6 \%$ des primären Lichts für die Detektion genutzt wird. Hieraus ergibt sich, dass ein Ion zum sicheren Nachweis ca. 800 Photonen in der Szintillatorfolie erzeugen muss. Wenn die Anzahl der Photonen, die ein Ion einer bestimmten Energie im Szintillator erzeugt, bekannt ist, kann die notwendige Dicke der Szintllatorfolie berechnet werden.

Für die Ausbeute an Photonen bei verschiedenen Energien und Teilchen gibt der Hersteller Bicron die Kurven aus Abbildung 5.7 an. Eine Parametrisierung der

\footnotetext{
${ }^{3}$ Hamamatsu Photonics, Tokyo, Japan.
} 


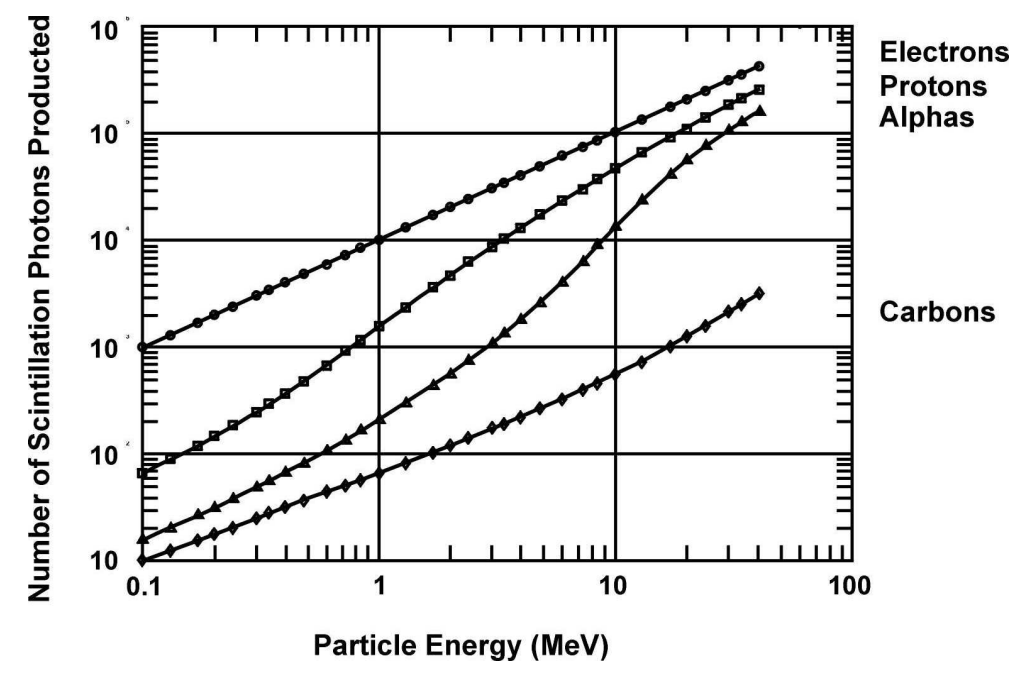

Abbildung 5.7: Anzahl der erzeugten Photonen für verschiedene Teilchensorten als Funktion der Teilchenenergie (nach [47]).

Lichtausbeute für Protonen im Szintillator des Typs NE-102A produkt von BC-400, ist bei R. Madey et al. zu finden [48]. Sie lautet:

$$
E_{e}=-8,0\left[1-\exp \left(-\frac{\left(E_{p} / E_{0}\right)^{0,9}}{10}\right)\right]+0,95 E_{p},
$$

mit $E_{0}=1 \mathrm{MeV}$. Diese empirische Formel rechnet eine Protonenenergie $E_{p}$ in eine Elektronenenergie $E_{e}$ um. Die Photonenausbeute für Elektronen liegt für alle Energien bei $10^{4} \mathrm{MeV}^{-1}$. Um die Ausbeute in einer dünnen Folie zu berechnen, muss man nur die Differenz zwischen den Energien eines einlaufenden und eines transmittierten Teilchens bilden.

Zur Abschätzung der Lichtausbeute der $\alpha$-Teilchen wurde das Programm NRESP4 [49] verwendet. Dieses berechnet zwar die Lichtausbeute eines NE-213 Szintillators, für eine Abschätzung stimmt diese aber mit der von BC-400 hinreichend überein, wie die Vergleichsdaten dieser beiden Szintillatoren zeigen [50]. Tabelle 5.4 bietet eine Übersicht der zu erwartenden Lichtausbeuten in einer Schichtdicke von $10 \mu m$ BC-400.

Aus Tabelle 5.4 ergibt sich, dass $\alpha$-Teilchen mit einem $10 \mu m$ dünnen Szintillator nachgewiesen werden können, während für Protonen $20 \mu \mathrm{m}$ bis $40 \mu \mathrm{m}$ notwendig sind. Am PTB Ionenstrahl werden Szintillatorfolien mit Dicken von $10 \mu \mathrm{m}, 20 \mu \mathrm{m}$ und $30 \mu m$ vorgehalten, so dass die Ionen der verschiedenen Energien und Sorten

\footnotetext{
${ }^{4}$ Nuclear Enterprises Ltd., UK.
} 


\begin{tabular}{|l|r|r|}
\hline Energie & \multicolumn{2}{|c|}{ Photonenausbeute } \\
$\mathrm{MeV}$ & Protonen & $\alpha$-Teilchen \\
\hline 3 & 550 & 1350 \\
5 & 450 & 1650 \\
8 & 400 & 1650 \\
10 & 350 & 1400 \\
12 & 300 & 1150 \\
15 & 250 & 1050 \\
20 & 220 & k.A. \\
\hline
\end{tabular}

Tabelle 5.4: Anzahl der erzeugten Photonen pro Ion in $10 \mu m$ BC-400 Folie.

nachgewiesen werden können. Da Protonen von $10 \mathrm{MeV}$ bereits eine Reichweite von 1,2 $\mathrm{mm}$ in Wasser haben, wird es bei hochenergetischen Protonen in vielen Fällen möglich sein, die Teilchendetektion hinter der Probe durchzuführen.

Die Sensitivität des Photomultipliers auf einzelne Photonen fordert die möglichst vollständige Abschirmung des Experimentierplatzes gegenüber externem Licht. Das wird durch einen umlaufenden lichtdichten Vorhang erreicht. Da die Verdunkelung nicht perfekt ist, kann es vorkommen, dass mehrere Restlichtphotonen gleichzeitig auf den Photomultiplier auftreffen und ein Signal verursachen, dass als Teilchendurchgang interpretiert werden muss. Die Rate solcher Ereignisse lässt sich leicht bestimmen, wenn man eine Messung ohne Ionnestrahl durchführt. Bei den bisherigen Messungen lag sie bei ca. $0,1 \mathrm{~s}^{-1}$. Eine ausführlichere Betrachtung und Beispiele für Spektren von PMT-Signalen sind in Kapitel 6.2.3 zu finden.

Der Photomultiplier ist in einem Gehäuse untergebracht, das auf dem Objektivrevolver des Mikroskops montiert wird und anstelle der Mikroskopobjektive in den Strahlengang geschwenkt werden kann.

\subsection{Streuung der Ionen nach dem Austritt aus dem Va- kuum}

In Kapitel 3.3 wurde die Streuung am Vakuumfenster unter den parasitären Aberrationen aufgeführt. Bei optimaler optischer Abbildung kann sie der limitierende Faktor für die erreichbare räumliche Auflösung sein. Wie in Abbildung $5.6 \mathrm{zu}$ sehen ist, werden die Ionen an folgenden Elementen gestreut: 
- Folie des Vakuumfensters

- Luftspalt zwischen Vakuumfenster und Probe

- Szintillatorfolie

- Trägerfolie

Den wesentlichen Anteil an der Aufstreuung des Ionenstrahls am Ort der Probe liefert die Folie des Vakuumfensters, da sie am weitesten von der zu bestrahlenden Probe entfernt ist. Daher müssen die Dicke der Folie und die Größe des Luftspaltes minimiert werden. Hingegen wird die Dicke des Szintillators von der Teilchenenergie und -sorte vorgegeben. Für die Trägerfolie stehen Eigenschaften wie Verträglichkeit der Zellen und Anwachsrate im Vordergrund. Der Luftspalt trägt zwar kaum zur Aufstreuung des Strahls bei, er kann aber selbst bei kleinen Streuwinkeln in der Fensterfolie eine große Ortsabweichung am Target bewirken. Wegen der Durchbiegung der Fensterfolie aufgrund des Luftdruckes ist er allerdings unvermeidlich. Deshalb muss ein möglichst kleines Vakuumfenster verwendet werden. Andererseits muss sichergestellt sein, dass der Strahl das Vakuumfenster trifft, da die Strahldiagnose auf dem Szintillationslicht beruht. Es kann also unter Umständen notwendig sein, den Strahl zunächst mit einem großen Austrittsfenster zu justieren und dann ein kleineres Fenster einzubauen, um Experimente mit hoher Ortsauflösung durchzuführen. Es wurden daher verschiedene Vakuumfenster mit Bohrungen zwischen $2 \mathrm{~mm}$ und 0,25 $\mathrm{mm}$ hergestellt, die sich leicht austauschen lassen.

Die Größe des Luftspaltes kann mithilfe des Mikroskops abgeschätzt werden, wenn man die Differenz der Objektivpositionen bei scharf eingestellter Fensterfolie und scharf eingestelltem Bestrahlungsobjekt misst. Für ein Fenster mit $2 \mathrm{~mm}$ Durchmesser kann der Luftspalt bis zu $300 \mu m$ betragen. Beim kleinsten Vakuumfenster von $250 \mu \mathrm{m}$ Durchmesser wurden ca. $30 \mu \mathrm{m}$ Luftspaltdicke gemessen. Die Durchbiegung der Fensterfolie kann von vielen Faktoren, wie Vorspannung, Temperatur oder Dauer der Benutzung abhängen. Es wurden daher Luftspaltdicken von $10 \mu \mathrm{m}$ bis $50 \mu \mathrm{m}$ angenommen und die resultierende Aufstreuung des Strahls mit dem Programm SRIM [41] für verschiedene Geometrien bei verschiedenen Teilchenenergien berechnet. Die Ergebnisse sind in der Tabelle 5.5 zusammengefasst. Es zeigt sich, dass die Ortsauflösung bei niedrigen Teilchenenergien durch die Winkelaufstreuung im Vakuumfenster begrenzt ist, da die optische Abbildung kleinere Strahldurchmesser zulässt (vgl. Tab. 3.2). Es könnte daher notwendig werden, den störenden Einfluss des Vakuumfensters weiter zu minimieren. 


\begin{tabular}{|c|c|c|c|c|c|c|c|}
\hline \multirow{2}{*}{\multicolumn{2}{|c|}{$\begin{array}{l}\text { Foliendicke } \\
\text { Luftspalt / } \mu m\end{array}$}} & \multicolumn{3}{|c|}{$4 \mu m$} & \multicolumn{3}{|l|}{$8 \mu m$} \\
\hline & & 10 & 20 & 50 & 10 & 20 & 50 \\
\hline \multicolumn{8}{|c|}{ Protonen (25 $\mu m$ Szintillator) } \\
\hline \multicolumn{2}{|c|}{$\begin{array}{l}\text { Energie / MeV } \\
\text { initial } \text { final (ca.) }\end{array}$} & \multicolumn{3}{|c|}{$\begin{array}{l}\text { Durchmesser } \\
\qquad / \mu m\end{array}$} & \multicolumn{3}{|c|}{$\begin{array}{l}\text { Durchmesser } \\
\qquad / \mu m\end{array}$} \\
\hline 3,4 & 3 & 1,26 & 1,36 & 1,73 & 1,47 & 1,72 & 2,38 \\
\hline 8,3 & 8 & 0,49 & 0,54 & 0,89 & 0,59 & 0,66 & 0,94 \\
\hline 15,1 & 15 & 0,26 & 0,28 & 0,35 & 0,31 & 0,36 & 0,49 \\
\hline \multicolumn{8}{|c|}{$\alpha$-Teilchen (10 $\mu m$ Szintillator) } \\
\hline \multicolumn{2}{|c|}{$\begin{array}{l}\text { Energie / MeV } \\
\text { initial } \text { final (ca.) }\end{array}$} & \multicolumn{3}{|c|}{$\begin{array}{c}\text { Durchmesser } \\
/ \mu m\end{array}$} & \multicolumn{3}{|c|}{$\begin{array}{l}\text { Durchmesser } \\
\qquad / \mu m\end{array}$} \\
\hline 5,6 & 3 & 0,89 & 1,05 & 1,62 & 1,26 & 1,58 & 2,59 \\
\hline 9,5 & 8 & 0,46 & 0,59 & 0,89 & 0,63 & 0,83 & 1,37 \\
\hline 16,0 & 15 & 0,26 & 0,32 & 0,51 & 0,36 & 0,46 & 0,77 \\
\hline
\end{tabular}

Tabelle 5.5: Strahlaufweitung durch Kleinwinkelstreuung am Vakuumfenster und an der Szintillatorfolie. Angegeben ist jeweils der Durchmesser von $90 \%$ Strahlintensität am Ort der Probe. Die Werte wurden mit dem Programm TRIM [41] unter der Vorraussetzung berechnet, dass der Strahl beim Austritt aus dem Vakuum eine verschwindende Ausdehnung und Divergenz besitzt.

\subsection{Fenstermechanik}

Aus Kapitel 5.3 geht hervor, dass der Abstand zwischen Vakuumfenster und Probe minimiert werden muss. Eine optimale Ortsauflösung wird erreicht, wenn die zu bestrahlende Probe und das Vakuumfenster mechanischen Kontakt haben. Andererseits muss die Probe transversal verschoben werden. Um die Fensterfolie nicht zu beschädigen, wird das Vakuumfenster vor jeder Bewegung des Mikropositioniertisches um einige Zehntel Millimeter nach oben bewegt und vor der erneuten Bestrahlung wieder gesenkt. Durch diese Prozedur wird die Bestrahlungsgeschwindigkeit zum jetzigen Zeitpunkt auf ca. 20 Positionen pro Minute begrenzt. Bei der Bewegung des Vakuumfensters ist es wichtig, dass sowohl die vertikale als auch die transversale Position genau reproduziert werden. Es wurde deshalb ein Rahmen konstruiert, der mithilfe von Kreuzrollenführungen die genaue und verschleißarme Bewegung des Vakuumfensters ermöglicht. Eine schematische Zeichnung der mechanischen Vorrichtung zur vertikalen Verschiebung des Vakuumfensters ist in Abbildung 5.8 dargestellt. 


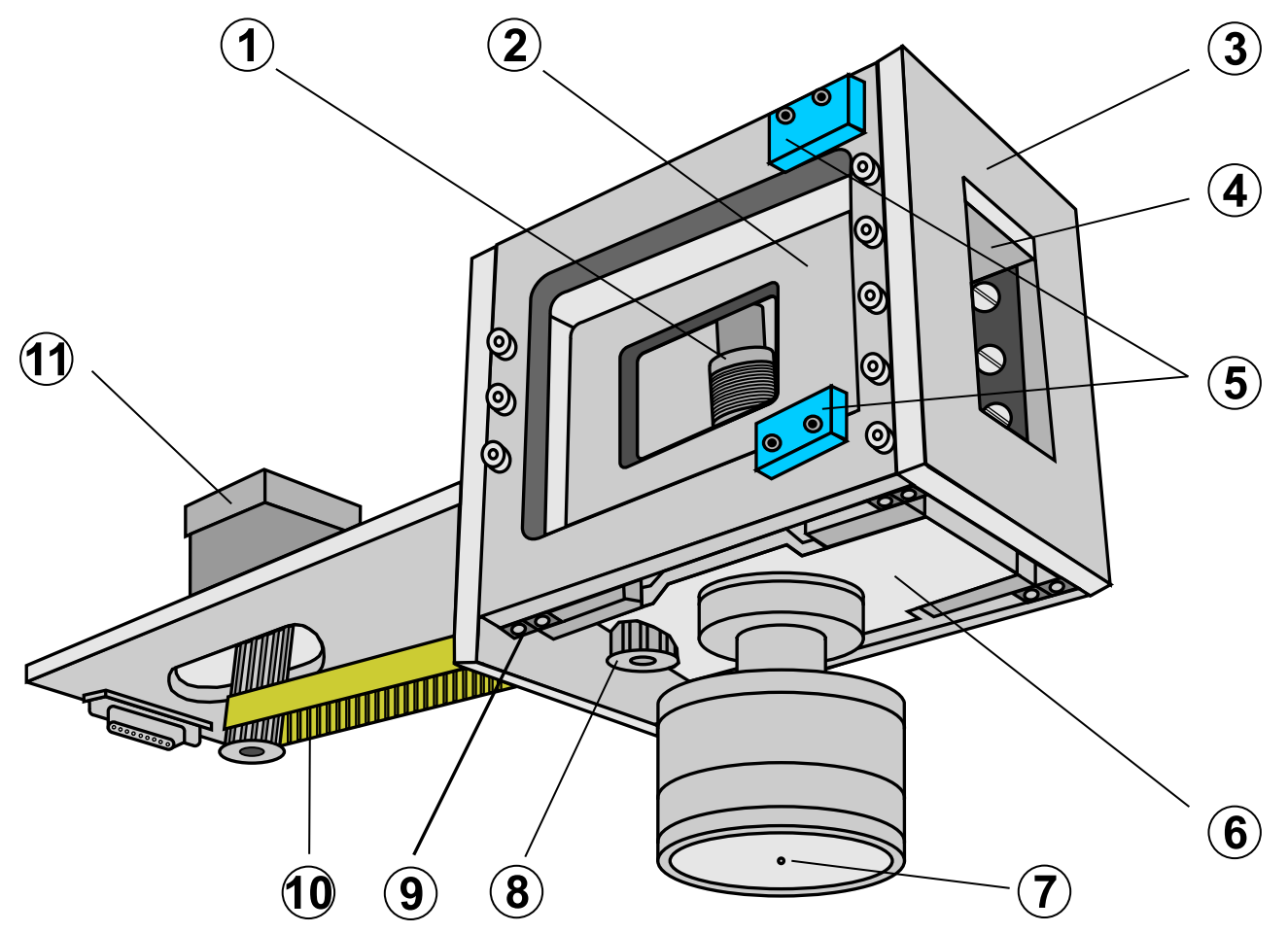

Abbildung 5.8: Mechanik zur vertikalen Positionierung des Vakuumfensters.

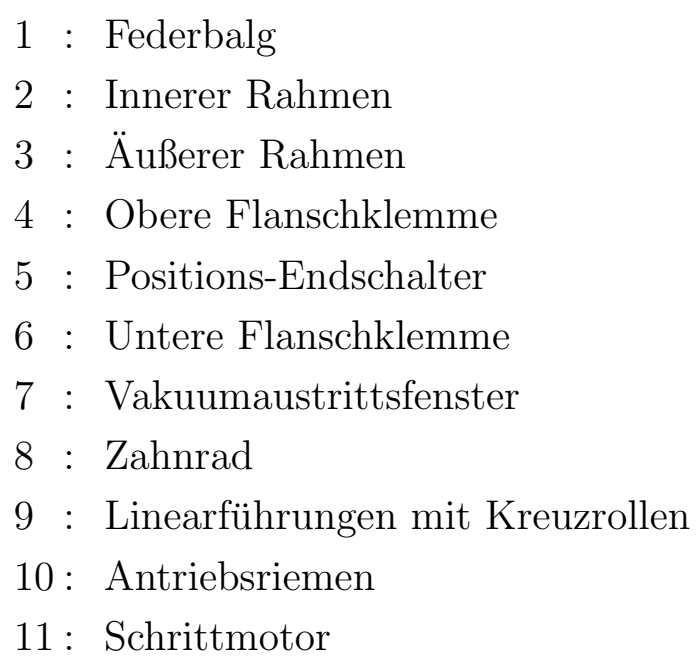

Um die Probe keinen mechanischen Belastungen auszusetzen und als Sicherung gegen Fehlbedienungen ist sie mit Linearführungen federnd gelagert. Die vertikale Position des Fensters wird mit einer Genauigkeit von einigen Mikrometern reproduziert. 


\subsection{Ablaufsteuerung}

Bei der Bestrahlung von Zellen muss eine Reihe von Komponenten in einer linearen Prozedur logisch miteinander verknüpft werden. Dies geschieht mittels eines PC, der die Komponenten via serieller Schnittstelle anspricht. Die Bestrahlung einer Position dauert zur Zeit ca. 3 Sekunden, so dass diese Art der Ansteuerung nicht zeitkritisch ist. Eine Ausnahme bildet die Schaltung des elektrostatischen Deflektors. Dieser wird von einem TTL-Signal angesteuert, das den Zustand des Zählers anzeigt (vgl. Abschnitt 4.2).

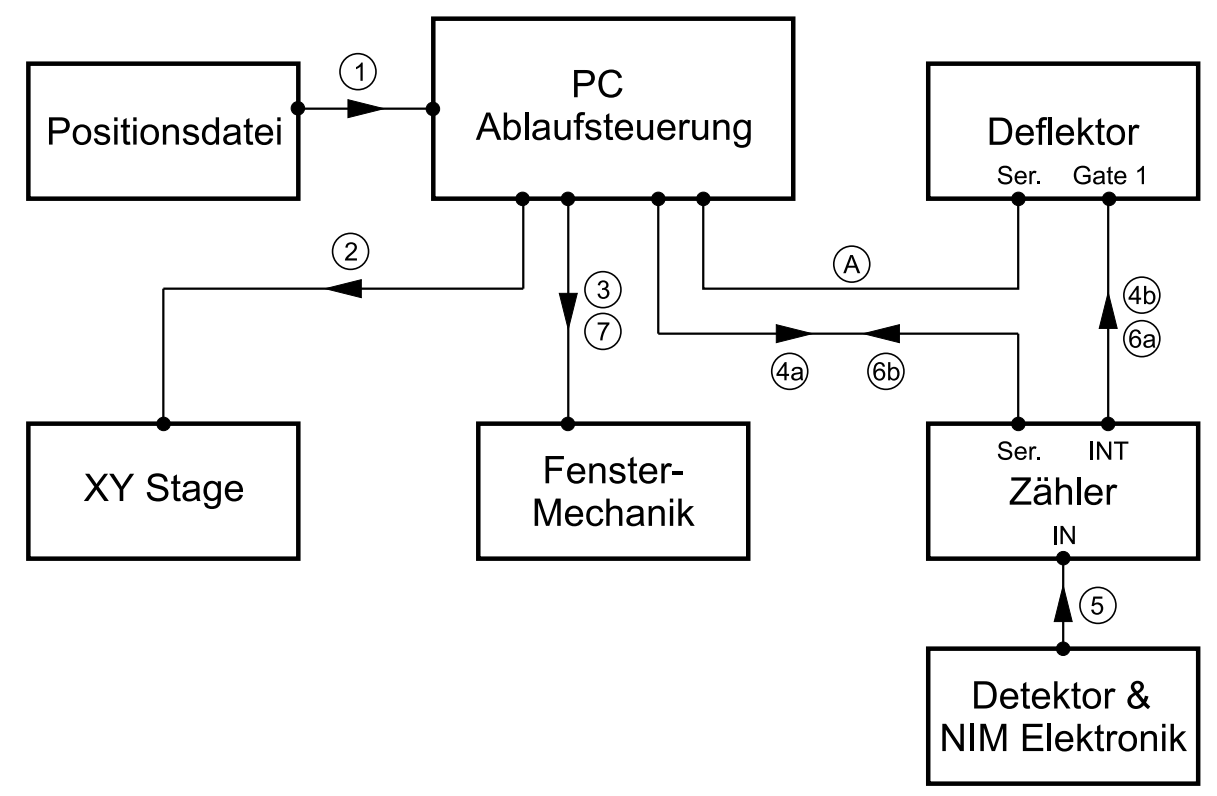

Abbildung 5.9: Darstellung der Bestrahlungsprozedur.

Die Bestrahlungsprozedur besteht aus folgenden Schritten:

1) Aus einer Positionsdatei, in der die Stage-Koordinaten der Targets gespeichert sind, wird ein Wertepaar eingelesen.

2) Der Mikropositioniertisch (XY-Stage) wird auf den aktuellen Wert gefahren.

3) Das Vakuumfenster fährt auf Kontakt zur Probe.

4a) Der Zähler der NIM-Elektronik wird aktiviert.

4b) Der Zustandsindikator des Zählers (Intervall) schaltet den Deflektor auf Durchgang. 
5) Die Detektorelektronik zählt die einlaufenden Teilchen.

6a) Wenn der voreingestellte Wert (Preset) erreicht ist, stoppt der Zähler und schaltet den Deflektor auf Hochspannung.

6b) Der PC erhält das Signal, dass die Bestrahlung dieser Position beendet ist.

7) Das Vakuumfenster wird angehoben.

Wiederholung der Schritte 1) bis 7) bei der nächsten Bestrahlungsposition.

Das zeitlimitierende Element ist die Fenstermechanik, die für beide Bewegungen zusammen (Schritt 3 und 5) ca. 2,5 Sekunden benötigt. Die eigentliche Bestrahlung dauert je nach Teilchenrate und eingestellter Anzahl nur einige Hundertstel bis Zehntel Sekunden. Da kleine Areale von wenigen Quadratmillimetern bestrahlt werden, ist die transversale Probenpositionierung ebenfalls in weniger als einer Sekunde durchzuführen. Die serielle Übertragung der Daten vom und zum PC dauert jeweils ca. 10 ms. Die Implementierung der Ablaufsteuerung erfolgte durch einen Ingenieur des Fachlaboratoriums.

\subsection{Zellerkennung und Zellpositionierung}

Zur Bestrahlung muss die Position jeder einzelnen Zelle bestimmt werden. Hierfür werden die Zellen auf einen eigens konstruierten Bestrahlungsträger (s. Abb. 5.10) aufgebracht. Dann wird die gesamte Fläche, in der sich die Zellen befinden, mit der CCD-Kamera schrittweise erfasst (scannen). Es wird die Pixelposition jeder Zelle im jeweiligen Bild bestimmt. Die Position des Ionenstrahls im Kamerabild wird entweder zuvor oder nach dem Scannen mit einem Szintillator (YAG oder BC-400) ebenfalls bestimmt. Durch Subtraktion der Pixelkoordinaten der Zellen und des Ionenstrahls kann ein Fahrweg errechnet werden, den der Mikropoitioniertisch ausgehend von der Scanposition zurücklegen muss, um die Zelle an die Position des Ionenstrahls zu bringen. Alle Bestrahlungspositionen werden in einer Datei gespeichert. Mithilfe der Ablaufsteuerung (s. Abschnitt 5.5) können dann alle Positionen mit der gewünschten Anzahl von Ionen bestrahlt werden. Während des Scannens des Bestrahlungsträgers ist der Ionenstrahl selbstverständlich ausgeschaltet. Während der eigentlichen Bestrahlung gibt es keine Möglichkeit zur optischen Kontrolle, da der Experimentierplatz zur Ionendetektion vollständig 


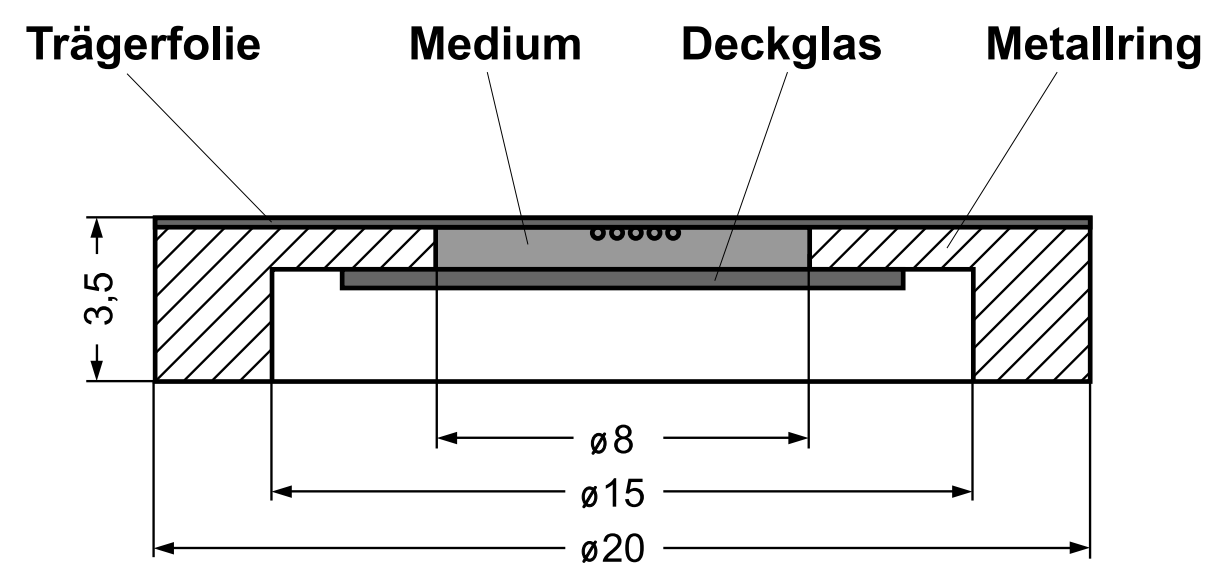

Abbildung 5.10: Schematischer Schnitt durch den Bestrahlungsträger. Die Zellen sind auf einen kleinen Bereich in dessen Mitte beschränkt.

abgedunkelt sein muss und zudem an der Stelle des Mikroskopobjektivs der Photomultiplier angebracht ist.

Als Bestrahlungsträger dient ein Ring aus Edelstahl für medizinische Anwendungen, dessen glatte Seite mit einer 12,5 $\mu \mathrm{m}$ starken Biofolie 5 bespannt ist (s. Abb. 5.10). Wenn die Zellen sich auf der hydrophilen Oberfläche der Biofolie festgeheftet haben, wird das zylindrische Volumen mit $50 \mu \mathrm{l}$ Nährmedium gefüllt und in einem mit $\mathrm{CO}_{2}$ begasbaren Brutschrank bei $37^{\circ} \mathrm{C}$ inkubiert. Eine Stunde vor der Zellpositionierung und der nachfolgenden Exposition mit abgezählten Ionen wird das Medium ausgetauscht gegen anderes Medium, das für die Erkennung der Zellkerne mit $2 \mu M$ des Hoechst Fluoreszenzfarbstoffes 33258 versetzt ist. Dieser Farbstoff färbt nur die Zellkerne und ist in dieser Konzentration praktisch nicht zytotoxisch. Nach der einstündigen Anfärbung wird das mit Hoechst 33258 versetzte Nährmedium gegen $50 \mu l$ frisches Nährmedium mit $20 \mu M$ HEPES ersetzt. Der HEPES-Zusatz erlaubt es, die Zellen für einige Stunden auch ohne $\mathrm{CO}_{2^{-}}$ Begasung bei einem konstanten pH-Wert von $7 \mathrm{zu}$ halten. Das Volumen wird mit einem Deckgläschen abgedeckt, das durch Adhäsion auch bei umgedrehtem Metallring haftet. Der Träger wird auf dem Mikropositioniertisch so befestigt, dass die mit der Biofolie bespannte Seite des Trägers nach oben zeigt. Durch Anregung bei $365 \mathrm{~nm}$ fluoreszieren die Zellkerne im blauen Bereich (Emission $420 \mathrm{~nm}$ ), so dass ihre Positionen im Kamerabild genau festgelegt werden können. Die Zellen werden mit Hilfe des Fluoreszenz-Umkehrmikroskops von unten durch das Deckgläschen $(0,13 \mathrm{~mm})$ und durch die $1 \mathrm{~mm}$ starke Mediumschicht betrachtet.

\footnotetext{
${ }^{5}$ Vivascience AG, Hannover.
} 
Hierzu wird ein Mikroskopobjektiv mit großem Arbeitsabstand und Korrektur der Deckglasdicke verwendet (Zeiss LD Achroplan ${ }^{\circledR}$ 20x Korr). Die Auflichtlampe des Mikroskops ist mit einer mechanischen Schließvorrichtung ausgestattet, so dass die Zellen nur während der Belichtungszeit der Kamera (ca. 0,1 s) beleuchtet werden.

Die oben beschriebene Präparationstechnik wurde von Professor Frankenberg vom Institut für Klinische Strahlenbiologie und Klinische Strahlenphysik entwickelt und getestet und für die ersten Bestrahlungsexperimente von humane Fibroblasten verwendet. Eine Modifikation der Präparation kann je nach verwendetem Zelltyp und gemessenem Endpunkt notwendig sein. Selbstverständlich muss der Einfluss der Präparationstechnik und der Umgebungsparameter in Kontrollen ausgiebig untersucht werden, um aussagekräftige Resultate bei der Bestrahlung zu erzielen.

In Zukunft soll der Zellträger temperiert werden, da die Umgebungstemperatur während des Scannens und der Bestrahlungen bei der Untersuchung von enzymatischen Reparaturvorgängen eine wichtige Rolle spielt. Um die Temperaturabhängigkeit der Reparatur der DNA-Doppelstrangbrüche zu untersuchen, ist eine Temperierung des Zellträgers herab bis zu $4^{\circ} \mathrm{C}$ notwendig. Für andere Experimente kann es hingegen sinnvoll sein, die Zellen ständig bei $37^{\circ} \mathrm{C}$ zu halten, um sie keinem zusätzlichen thermischen Stress auszusetzen.

Die Anzahl der Zellen, die auf einem Zellträger bestrahlt werden können, hängt von der Geschwindigkeit der Zellerkennung und der Art des Bestrahlungsexperimentes ab. Für die Bestimmung des klonalen Zellüberlebens wurde eine Zahl von 50 bis 200 Zellen festgelegt. Diese Zahl wurde so gewählt, dass der Stichprobenumfang jeder Bestrahlung eine statistisch signifikante Aussage erlaubt, aber andererseits die Verweildauer der Zellen unter Bestrahlungsbedingungen nicht so lang ist, dass dies einen Einfluss auf das Zellüberleben hat. Damit beim Scannen des Trägers nicht hauptsächlich leere Bilder aufgenommen werden, ist eine Beschränkung der Zellen auf eine Fläche von wenigen Quadratmillimetern wünschenswert. Daher wird zu Beginn der Präparation nur wenig Medium $(7 \mu l)$ mit der gewünschten Anzahl von Zellen auf die Mitte des Trägers aufgebracht, so dass diese auf einen Bereich von ca. $3 \mathrm{~mm}$ Durchmesser beschränkt bleiben. Mit dem $\times 20$ Objektiv (Bildfeld $420 \times 330 \mu m^{2}$ ) sind ca. 8 Aufnahmen je Quadratmillimeter notwendig, so dass unter diesen Bedingungen zum Scannen ca. 80 bis 100 Aufnahmen gemacht werden müssen. 


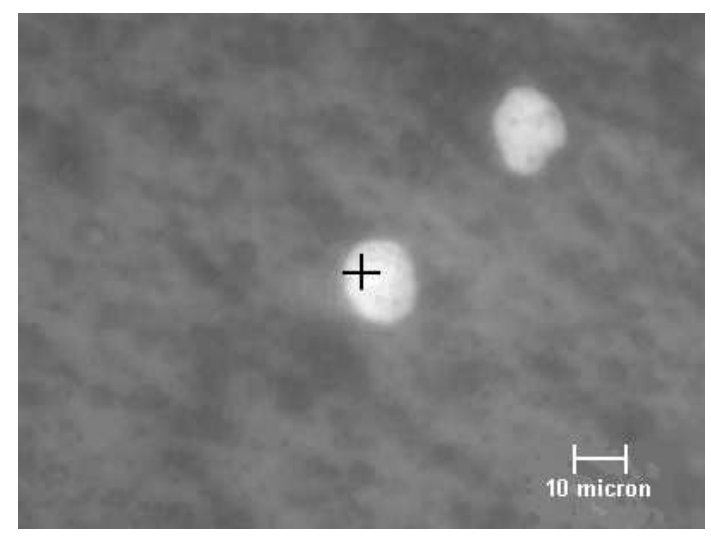

Abbildung 5.11: Repositionierte Zellen. Das Kreuz gibt die Sollposition an.

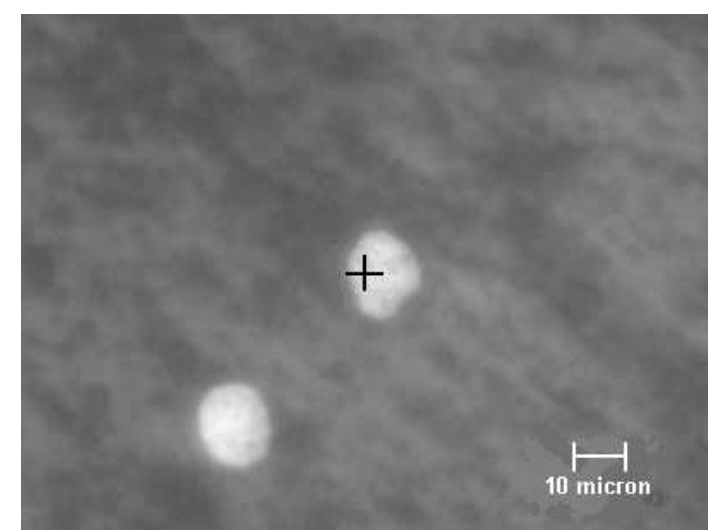

Abbildung 5.12: Die gleichen Zellen wie in Abb. 5.11, jedoch mit der anderen in der Sollposition.

Zur Überprüfung der zur Zeit erreichbaren Positioniergenauigkeit wurde ein Test durchgeführt, bei dem die Zellen nach der Positionierung nicht bestrahlt, sondern mit der Kamera aufgenommen wurden. Als Strahlposition im Kamerabild wurde willkürlich die Pixelposition $(600,500)$ festgelegt. Dann wurden die Zellen mithilfe des Mikropositioniertisches an ihre Sollposition gefahren. Anhand der Bilder kann dann abgezählt werden, wieviele der Zellen vom Mikro-Ionenstrahl getroffen worden wären. Zwei vergrößerte Bildausschnitte der repositionierten Zellen sind in den Abbildungen 5.11 und 5.12 dargestellt. Ein Test mit humanen Fibroblasten, deren Kerndurchmesser $10 \mu m$ bis $15 \mu m$ betrug, ergab, dass die Sollposition bei ca. $96 \%$ innerhalb der Zellkerne lag.

In Abbildung 5.13 sind die Ergebnisse der Repositionierungsmessung graphisch zusammengefasst. In dieser Messung zeigt sich eine systematische Abweichung von ca. $3 \mu \mathrm{m}$ bzw. $1 \mu \mathrm{m}$ und eine Breite der gemessenen Ortsverteilung von jeweils ca. $3 \mu \mathrm{m}$. Die Breite ist hauptsächlich durch die beschränkte Absolutgenauigkeit des Mikropositioniertisches bedingt. Diese wird ausführlicher in Abschnitt 5.6.1 behandelt.

Der Mittelwert, der deutlich von Null abweicht, stellt einen systematischen Fehler dar. Über seine Herkunft kann bisher noch keine verlässliche Aussage gemacht werden. Möglicherweise ist die Arretiervorrichtung des Mikroskoprevolvers dafür verantwortlich, da derzeit eine Berührung des Objektivs eine Verschiebung im Kamerabild verursachen kann. Das Gleiche gilt auch für die Befestigungsvorrichtung der Kamera. 


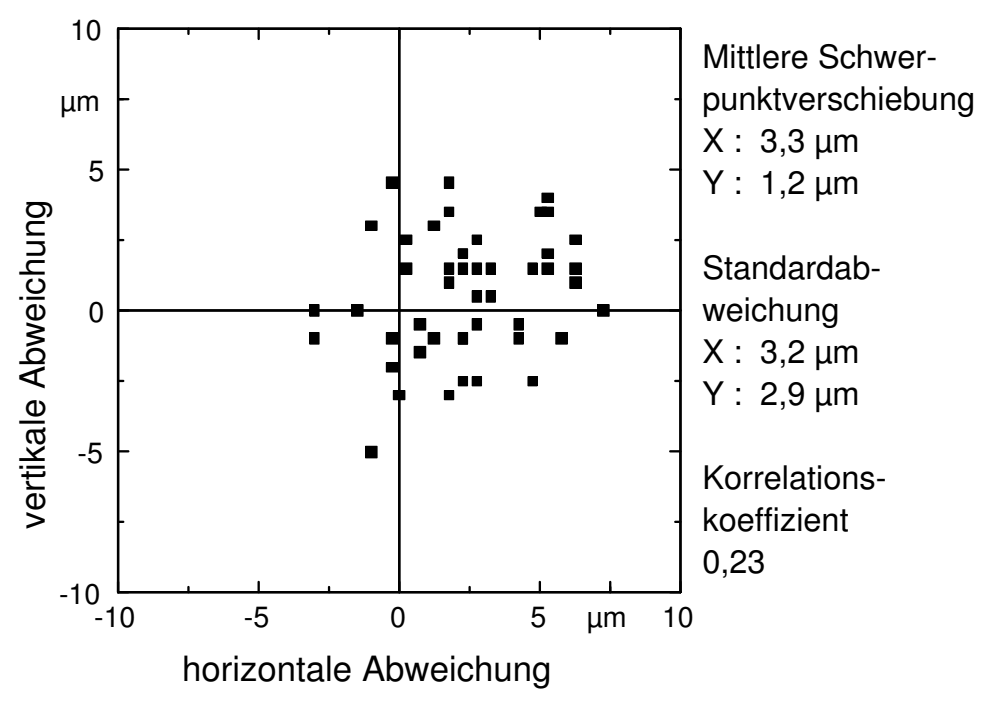

Abbildung 5.13: Horizontale und vertikale Abweichung der tatsächlichen Zellposition von der Sollposition.

Die bei den ersten Experimenten angewandte manuelle Prozedur zur Bestimmung der Zellschwerpunkte ist recht ungenau, fehleranfällig und arbeitsintensiv. Bei ihr wurden die Schwerpunkte der Zellen jeweils aufgrund des optischen Eindrucks mit einer Auflösung von nur einem Pixel bestimmt. Es ist deshalb notwendig, dass die Erkennung und Positionierung der Zellen in Zukunft durch eine automatische Prozedur erfolgen. Hierzu müssen die Aufnahmen durch geeignete Manipulationen und Filter binarisiert werden. Anschließend können die Parameter der Objekte vermessen und die richtigen Objekte aussortiert werden. Moderne Bildverarbeitungssoftware liefert hierfür die wesentlichen Hilfsmittel. Es ist aber zu beachten, dass die Prozedur flexibel genug sein muss, um auf eine Variation verschiedener Parameter, wie z.B. Kontrast, Objektgröße oder -form, reagieren zu können. Außerdem müssen in diese Prozedur die verschiedenen Hardware-Komponenten, wie CCD-Kamera, Mikropositioniertisch, Mikroskopshutter und Fenstermechanik eingebunden werden.

\subsubsection{Genauigkeit des Mikropositioniertisches}

Zur Repositionierung muss die Position einer Zelle im Kamerabild (Pixelkoordinaten) in einen Verfahrweg des Mikropositioniertisches umgerechnet werden. Hierzu ist eine hohe Genauigkeit und Verlässlichkeit der mechanischen Komponenten sowie der optischen Positionsbestimmung erforderlich. 
Grundlage für die Umrechnung sind zum einen die Pixelabmessungen. Der Hersteller gibt an, dass die Pixel quadratisch sind mit einer Kantenlänge von 6,8 $\mu \mathrm{m}$. Im Kamerabild ergibt die Messung mithilfe eines Objektmikrometers eine Pixelgröße im von $6,56 \times 6,56 \mu \mathrm{m}^{2}$. Die Abweichung von der physikalischen Pixelgröße wird durch die Optik der CCD-Kamera verursacht, die eine Abweichung der lichtoptischen Vergrösserung vom Sollwert 20 (Objektivvergrößerung) bewirkt. Nach bisherigen Messungen sind die Pixelabmessungen und die lichtoptische Vergrößerung über die gesamte Fläche des CCD-Chips hinreichend konstant, so dass keine Verzerrungen des Kamerabildes auftreten. In die Berechnung geht außerdem die Verkippung der CCD-Kamera gegenüber den Achsen des Mikropositioniertisches ein. Bisher wurde dieser über die Bewegung eines Referenzobjektes entlang einer Achse bestimmt. Für kleine Winkel $(\leq 10 \mathrm{mrad})$ ist dies beim derzeitigen Stand der Anlage ausreichend genau.

\begin{tabular}{|l|r|}
\hline Spindelsteigung & $1 \mathrm{~mm}$ \\
Auflösung & $0,025 \mu \mathrm{m}$ \\
Reproduzierbarkeit & $\leq 0,75 \mu \mathrm{m}$ \\
Genauigkeit & $\pm 3 \mu \mathrm{m}$ \\
\hline
\end{tabular}

Tabelle 5.6: Mechanische Parameter des Mikropositioniertisches [51].

Es hat sich herausgestellt, dass die Absolutgenauigkeit des Mikropositioniertisches der kritische Faktor ist. Der Hersteller gibt für die mechanischen Parameter die Werte aus Tabelle 5.6 an. Die Aufösung gibt die nominelle Schrittweite an. Die Reproduzierbarkeit bezeichnet die Schwankungsbreite, wenn ein und dieselbe nominelle Position mehrfach angesteuert wird. Sie wird z.B. durch Gewindegänge limitiert, die einen Fehler bei Richtungsumkehr verursachen. Die Genauigkeit bezeichnet die Übereinstimmung zwischen nomineller und tatsächlicher Position. Die nominelle Position wird durch Wahl eines Referenzpunktes und der Anzahl der gefahrenen Schritte bestimmt. Ihr liegt keine Messung zugrunde. Der im Vergleich zur Auflösung große Schwankungsbereich der Genauigkeit kommt durch Abweichung der lokalen Spindelsteigung vom Sollwert zustande.

In Abbildung 5.14 ist die Abweichung der Spindelsteigung für einen kleinen Bereich dargestellt. Es ist eine regelmäßige Schwankung, deren Periode mit der Spindelsteigung übereinstimmt. Die Messung wurde mit einem Laserinterferometer unter kontrollierten klimatischen Bedingungen durchgeführt. Da die Spindelsteigung einen systematischen Fehler hat, der offenbar hochgradig reproduzierbar ist, kann er durch eine Kalibrierung des Mikropositioniertisches reduziert 


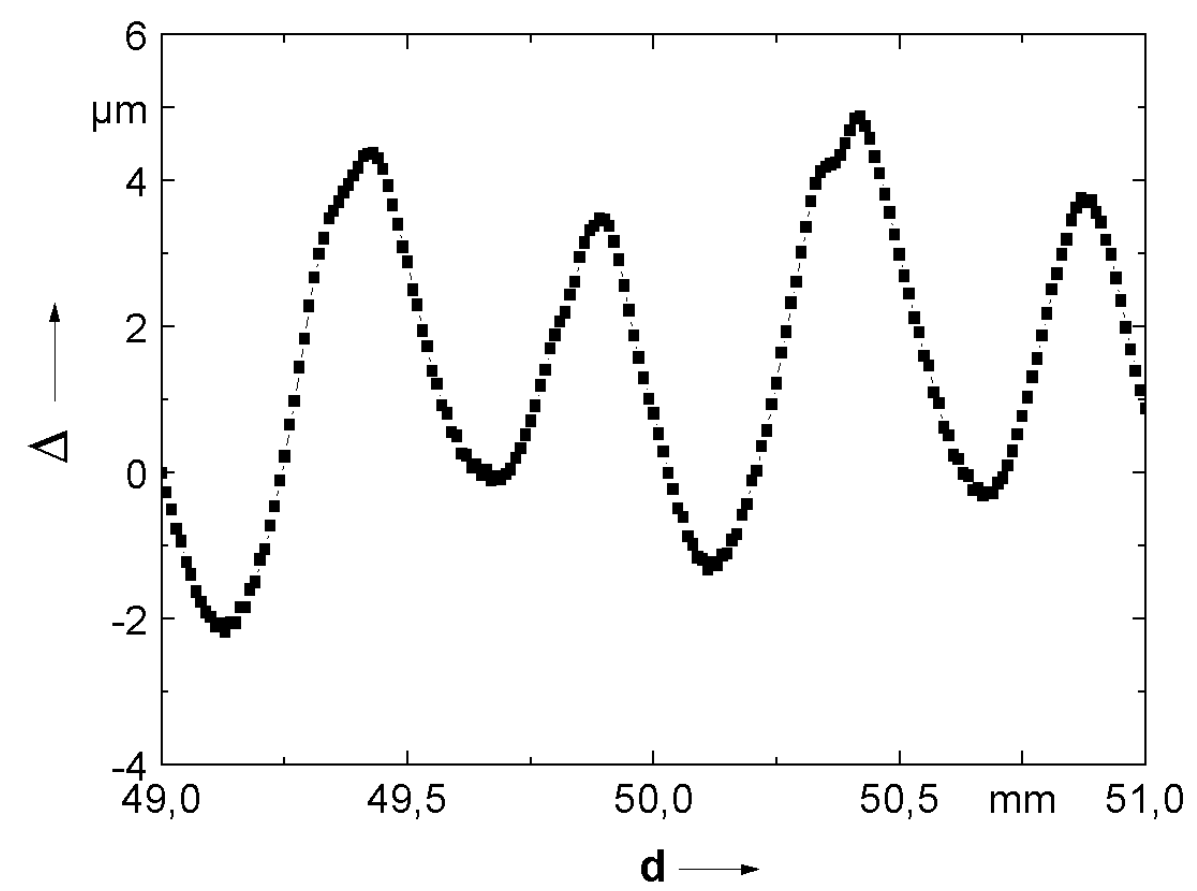

Abbildung 5.14: Differenz zwischen tatsächlicher und nomineller Position $(\Delta)$ in Abhängigkeit von der Position des Mikropositioniertisches (d). Die scheinbare Verschiebung der Kurve in den Bereich positiver Werte ist durch die willkürliche Anfangsphase verursacht.

werden. Hierbei wird der gesamte Fahrweg mit einem Laserinterferometer mit hoher Genauigkeit $(<0,1 \mu m)$ vermessen. In jedem Bestrahlungsexperiment kann dann den nominellen Positionen die tatsächliche zugeordnet werden. Die Klimatisierung und Klimadatenerfassung in der Experimentierhalle gewährleisten, dass unerwünschte thermische Ausdehnungen der mechanischen Komponenten nicht auftreten können. 


\section{Kapitel 6}

\section{Messungen der Strahlparameter}

\subsection{Fokussierung}

Die Einstellung der Abbildungseigenschaften erfolgt sukzessiv, da der Einzelteilchenstrahl, der für die biologischen Experimente gebraucht wird, für die Strahldiagnose ungeeignet ist. Es werden mindestens drei Schritte gemacht:

- Einstellung des Einschusses in Strahlrohr 4 mit dem Durchgangsloch der Objektblende (vgl. Abb. 4.2)

- Einfädelung des Folienstrahls auf das Vakuumfenster und anschließende grobe Einstellung der Fokussierung

- Optimierung der Abbildung des Mikrostrahls mit sukzessiver Verringerung der Strahldivergenz

Die Prozedur der Fokussierung beginnt mit der zentralen Einstellung des Strahls auf die Objektblende. An dieser ist ein Szintillationsschirm angebracht, mit dem man das Strahlprofil und die Strahllage an dieser Stelle abschätzen kann. Der zentrale Beschuss der Objektblende ist allerdings nicht gleichbedeutend mit dem geraden Einschuss in Strahlrohr 4. Deshalb wird der Strahl durch eine freie Bohrung geschossen und der einlaufende Strahl so modifiziert, dass eine zentrale Position des Strahls und ein maximaler Strahlstrom auf Schlitz 41 erreicht werden.

\subsubsection{Justierung des Folienstrahls}

Für die grobe Justierung des Strahls, insbesondere die Richtungskorrekturen durch Korrekturmagnete, wird dann der Folienstrahl (s. Abschnitt 4.1) verwendet. Anhand der Schlitzströme wird hierbei jeweils die Strahllage bestimmt. Erst wenn der Strahl mit dem YAG-Leuchtschirm und der Kamera visualisiert werden 


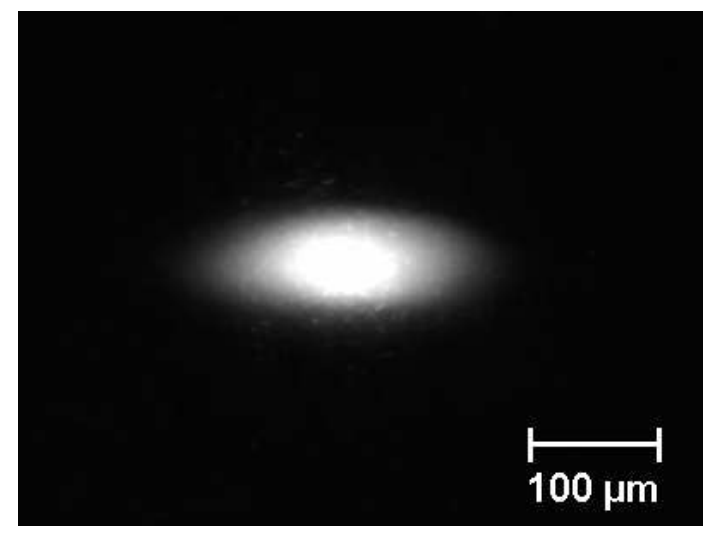

Abbildung 6.1: Bild des Folienstrahls bei optimaler Abbildung.

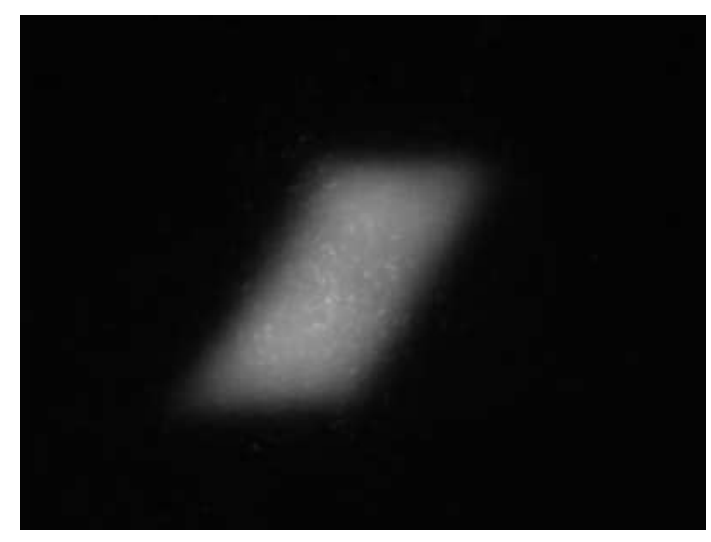

Abbildung 6.2: Bild des Folienstrahls bei nicht optimaler Abbildung.

kann, ist es möglich, die Strahleigenschaften mit den Quadrupolen zu optimieren. Die Einstellung des Dipolfeldes ist unproblematisch, da es wegen der genauen Messung des Magnetfeldes gut reproduzierbar eingestellt werden kann.

Anhand der Eigenschaften des Folienstrahls kann man die Abbildungseigenschaften (1. Ordnung) der Optik abschätzen, da hier Effekte höherer Ordnung kaum ins Gewicht fallen. Der Maßstab in axialer Richtung ist sofort ablesbar. Wie in Kapitel 3.4 dargestellt, wird eine Verkleinerung von etwa 20 eingestellt. Die Ortsdispersion kann abgeschätzt werden, indem die Schlitzweite im Analysiersystem geändert wird. Bei verschwindender Ortsdispersion $\left(R_{16}=0\right)$ wird dies keine Änderung der Ausdehnung in radialer Richtung bewirken. Ist dies der Fall, werden die Strahlausdehnungen durch die beiden Verkleinerungen $\left(R_{11}, R_{33}\right)$ bestimmt. Wenn der Strahl im Vakuumfenster liegt, dauert die Einstellung des Folienstrahls zumeist nur einige Minuten, da die Strahleigenschaften nicht sehr sensibel auf Änderungen der Quadrupolstärken reagieren.

\subsubsection{Optimierung des Mikrostrahls}

Wenn mit dem Folienstrahl zufriedenstellende Abbildungseigenschaften erreicht sind, werden die Magnete um einen kleinen Betrag korrigiert, welcher der Impulsdifferenz zwischen dem Folienstrahl und dem ungestreuten Strahls entspricht. Zumeist werden damit Abbildungseigenschaften erreicht, die in der Nähe des Optimums liegen.

Mit dem Mikrostrahl wird dann die Ortsdispersion überprüft. Hierzu wird der Schlitz 43 weit geöffnet $(\geq 1 \mathrm{~mm}$ ) und dann das Magnetfeld des Dipols ge- 


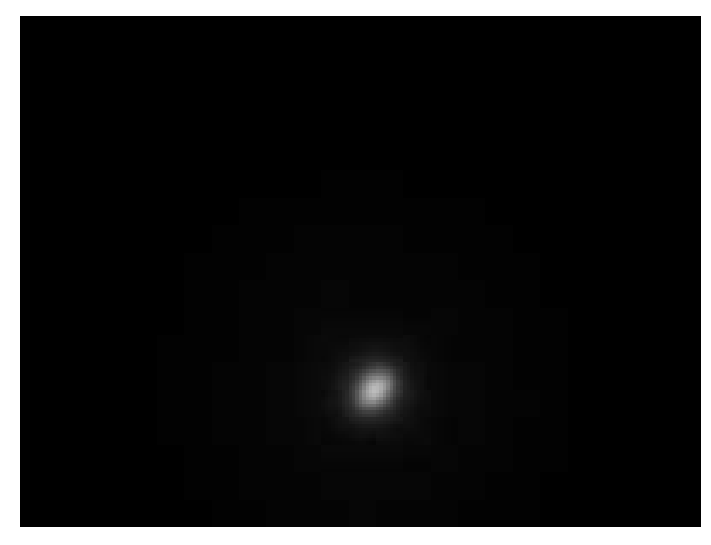

Abbildung 6.3: Strahlfleck bei Dipolfeld von $3349,30 G$.

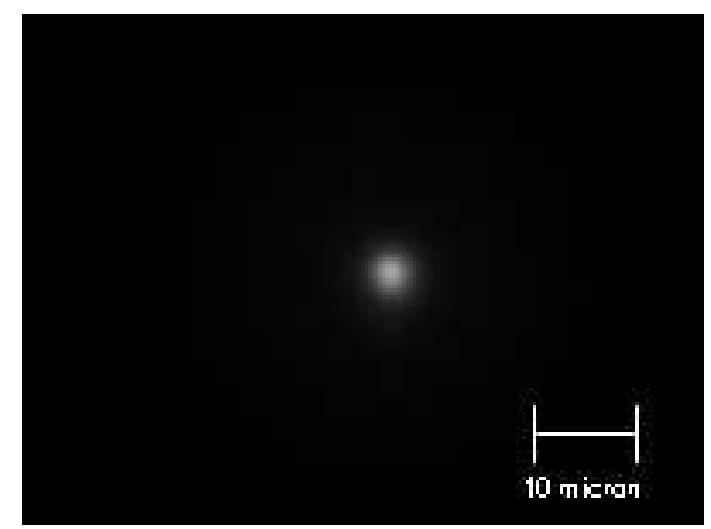

Abbildung 6.4: Strahlfleck bei Dipolfeld von 3347,36 G.

ringfügig variiert $\left(10^{-3}\right.$ relative Feldänderung). Bei verschwindender Ortsdispersion sollte es nicht möglich sein, die Position des Strahlflecks zu ändern. Die beiden Magnetfeldstärken aus den Abbildungen 6.3 und 6.4 entsprechen einem Unterschied im Ablenkwinkel von 0,3 mrad. Nach einer Driftstrecke von $4 \mathrm{~m}$ würde hieraus eine Ortsabweichung von $1,2 \mathrm{~mm}$ resultieren. Die tatsächliche räumliche Differenz in radialer Richtung beträgt ca. $1 \mu \mathrm{m}$. Daraus lässt sich eine Ortsdispersion von $R_{16} \leq 0,002 \mathrm{~mm} / \%$ abschätzen. Bemerkenswert ist, dass sich die Position des Strahls in axialer Richtung wesentlich stärker ändert. Dies deutet auf parasitäre Aberrationen hin, die durch eine Fehljustierung der Magnete verursacht werden. Dieses Problem wird in Abschnitt 6.1 .3 eingehender behandelt.

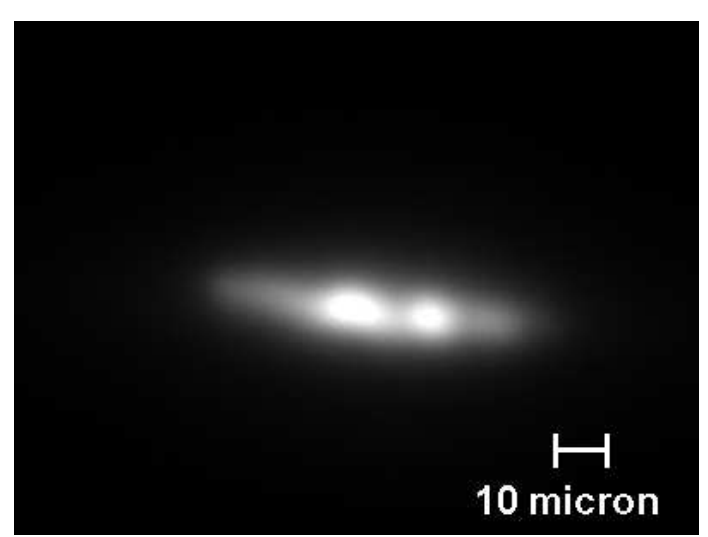

Abbildung 6.5: Horizontal getrennte Strahlflecken bei nicht verschwindender Ortsdispersion.

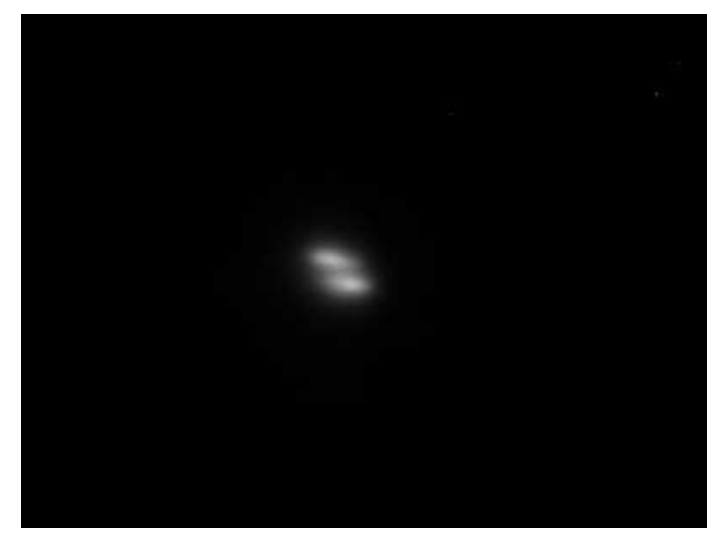

Abbildung 6.6: Horizontal und vertikal getrennte Strahlflecken durch Aberrationen.

Der VdG-Strahl hat die Eigenschaft, dass in ihm mehrere Energiezustände auftreten können, die sich um einige $k e V$ unterscheiden. Dadurch wird es möglich, 
die Größe der Ortsdispersion mit einer weiteren Methode abzuschätzen. Abbildung 6.5 zeigt einen Strahl, bei dem die Bedingung für eine Punkt zu Punkt Abbildung erfüllt ist, aber die Ortsdispersion offensichtlich nicht null ist. Bei einer Verringerung der Ortsdispersion werden die vorher separierten Strahlflecken auf denselben Ort abgebildet.

Die Impulsverteilung des VdG-Strahls ist aus früheren Testmessungen genau bekannt. Zu einem Zeitpunkt, als die Strahlführung des Mikro-Ionenstrahls noch nicht komplett war, wurde hierzu das Strahlprofil an der Stelle des Schlitzes 43 bestimmt. Wenn der Strahl an dieser Stelle die Bedingungen für die Punkt-zu-Punkt Abbildung erfüllt, ist das radiale Strahlprofil gegeben durch die Impulsverteilung der Ionen. Es zeigte sich, dass der VdG-Strahl durch die Regelung der Terminalspannung zwischen mehreren Energien mit einer unregelmäßigen Frequenz von einigen Hertz hin und her springt. Bei einer langen Kameraintegrationszeit $(>0,5 s)$ werden die verschiedenen Energiezustände als getrennte Strahlflecken abgebildet. Die einzelnen Zustände haben etwa eine relative Impulsbreite von 0,05 \% \% Zumeist lassen sich zwei oder drei Zustände unterscheiden, deren Impulsdifferenz ebenfalls ca. 0,05\% beträgt, so dass eine Impulsbreite von insgesamt 0,15\% \% resultiert. Aus diesen Werten lässt sich berechnen, dass die Ortsdispersion in Bild 6.5 ca. 0,2 mm/\% betrug. Die Abbildung von Ionen verschiedener Energien auf denselben Ort dient als Optimierungskriterium für die optische Abbildung.

\subsubsection{Auswirkungen von parasitären Aberrationen}

Durch den Einfluss der Ortsdispersion ist es verständlich, dass mehrere Strahlflecken auftreten können, die horizontal getrennt sind. Da in der Vertikalen keine Dispersion vorkommt, ist eine energieabhängige Abbildung in dieser Richtung in 1. Ordnung unmöglich. Tritt eine vertikale Trennung auf, so deutet dies auf eine chromatische Aberration hin. In Abbildung 6.6 ist ein solcher Fall dargestellt. Der vertikale Abstand beträgt hier ca. $5 \mu \mathrm{m}$. Bei einer Impulsdifferenz von 0,05\% und einer Divergenz geringer als 1 mrad ist dies nicht mit dem errechneten Koeffizienten $T_{346} \approx 10 \frac{\mu m}{\mathrm{mrad}_{00}}$ verträglich. Der Grund für die unerwartet hohe Aberration liegt in der Fehljustierung des horizontalen Quadrupoldubletts. Hierdurch erhöht sich die effektive Eingangsdivergenz des Strahls.

Das Problem der unzureichenden Justierung der horizontalen Strahlführung macht sich auch noch auf andere Weise bemerkbar. Bei einer Änderung der hori- 
zontalen Magnetströme verändert sich nicht nur die Form des Strahlflecks, sondern auch seine Lage. Die Quadrupole haben also einen ablenkenden Effekt. Dieser kann nur dann auftreten, wenn der Strahl sich nicht auf der Sollbahn befindet. Dieses Problem lässt sich derzeit nicht zufriedenstellend lösen, da die mechanische Justierung des horizontalen Dubletts keine höhere Genauigkeit als $\pm 1 \mathrm{~mm}$ zulässt. Bei Veränderung der Stärke des mikrofokussierenden Dubletts wird ebenfalls ein leichter Ablenk-Effekt beobachtet. Die Minimierung dieses Effektes kann als Kriterium für die Ausrichtung des Strahls entlang der Magnetachse dienen.

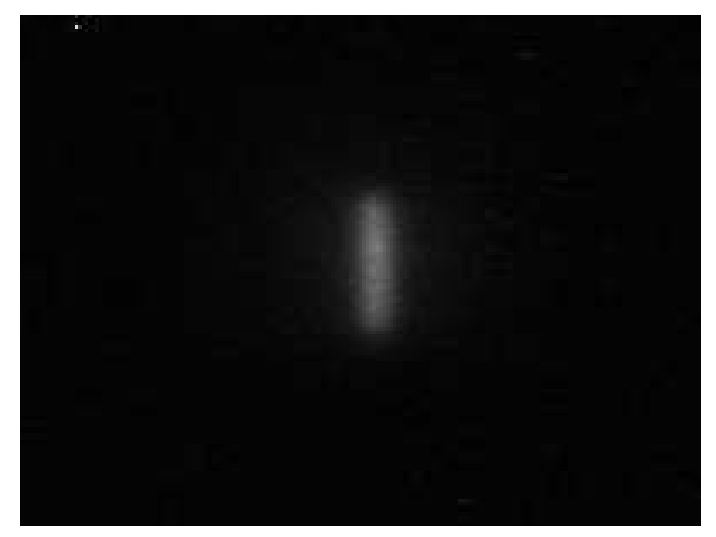

Abbildung 6.7: Strahlfleck bei offenen Divergenzschlitzen.

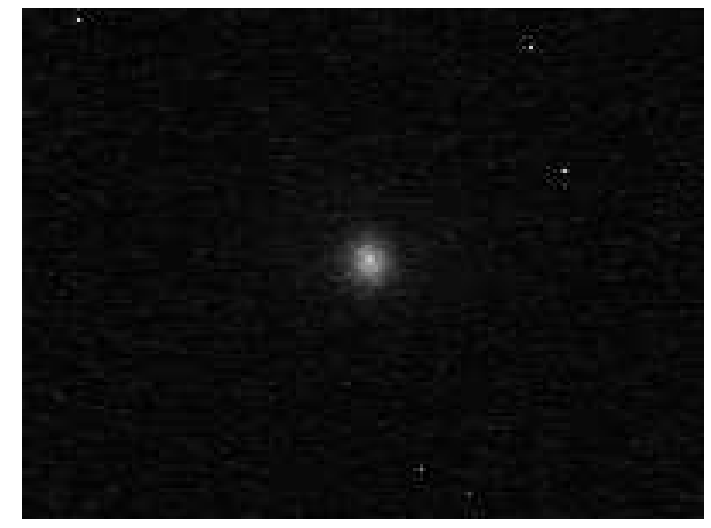

Abbildung 6.8: Strahlfleck bei eingeengten Divergenzschlitzen.

Der Einfluss der chromatischen Aberration ist auch in Abbildung 6.7 sichtbar. Hier ist aber zu beachten, dass es sich um einen $\alpha$-Teilchenstrahl aus dem Zyklotron handelt, dessen relative Impulsbreite erheblich größer ist als die des VdGStrahls. Sie kann je nach Einstellung der Strahlführung 1\% und mehr betragen.

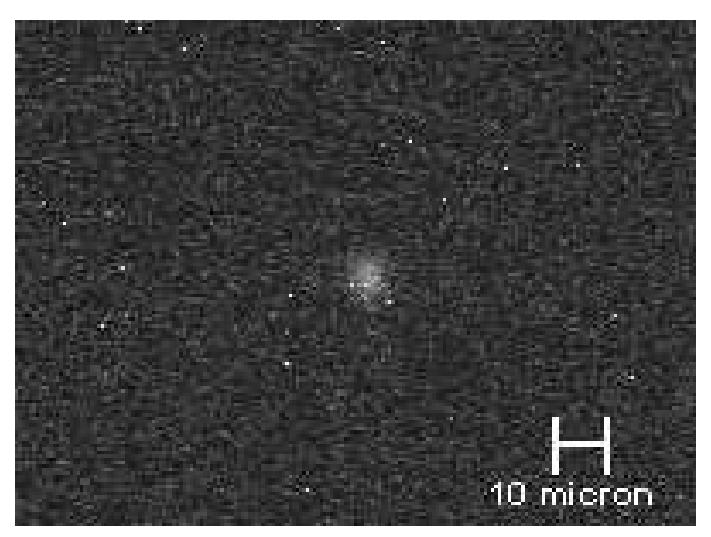

Abbildung 6.9: $8 \mathrm{MeV} \alpha$-Teilchen bei einer Rate von ca. $100 s^{-1}$ und bei 60 s Bildaufnahmezeit.

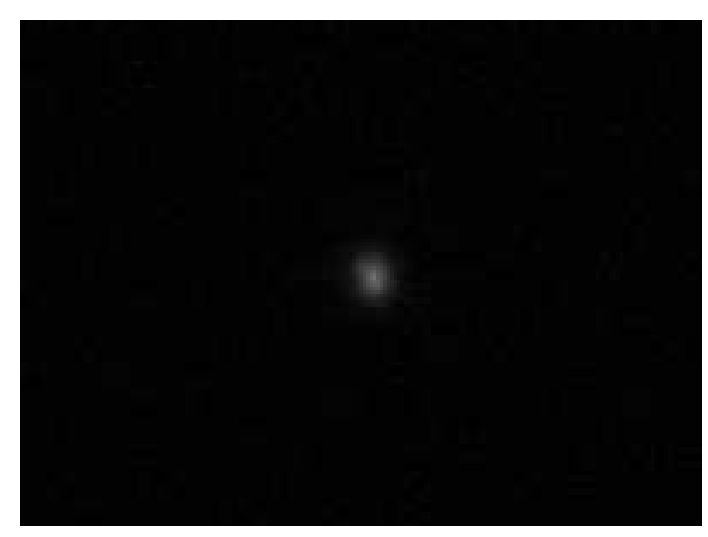

Abbildung 6.10: Das gleiches Bild wie in Abb. 6.9, nachdem das Dunkelbild (ohne Strahl) subtrahiert wurde. 
Große chromatische Aberrationen führen dazu, dass ein zufriedenstellend kleiner Strahlfleck ( $<3 \mu \mathrm{m}$ Durchmesser) erst bei stark eingeschränkter Strahldivergenz erreicht wird. Für die Experimente mit geringer Teilchenrate bedeutet dies keine Einschränkung, hat aber zur Folge, dass die Integrationszeiten der CCD-Kamera während der Fokussierprozedur unhandlich lang werden können (>30s). In Abbildung 6.9 ist ein Strahl zu sehen, dessen Intensität nur leicht über dem Restlicht aus unerwünschten Lichtquellen und dem Rauschen der Kamera liegt. Bei einem Experiment mit Zellen muss die Strahlposition mit einem BC-400 Szintillator, der eine wesentlich geringere Lichtintensität als YAG liefert, jedoch bestimmbar sein, so dass eine kurzfristige Erhöhung des Strahlstroms ohne Veränderung der Strahleigenschaften wünschenswert ist. Eine Optimierung der Justierung der Strahlführungselemente zur Verringerung der Aberrationen ist deshalb sinnvoll.

\subsection{Teilchendetektion}

\subsubsection{Spurdetektor}

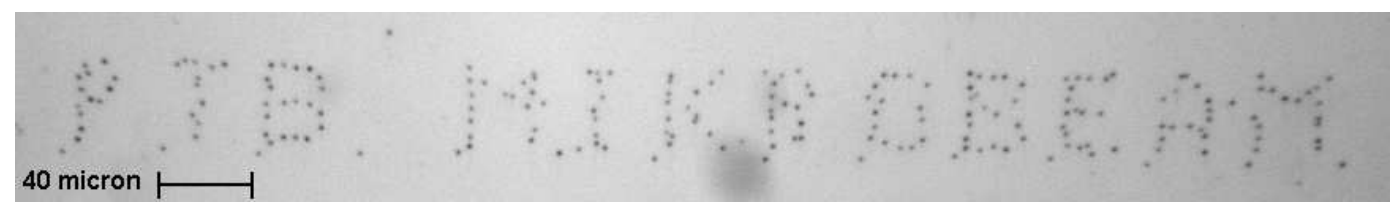

Abbildung 6.11: Schriftzug aus den Spuren einzelner Ionen auf CR39. In diesem Fall wurde nach dem Auftreffen eines einzelnen Ions der Spurdetektor jeweils um eine geringe Distanz verschoben, so dass sich der Schriftzug PTB MIKROBEAM ergibt.

Zur Überprüfung der Parameter des Mikro-Ionenstrahls wird der Spurdetektor CR391 [52] verwendet. Mit diesem Material lassen sich die Spuren einzelner Ionen durch ein Ätzverfahren sichtbar machen. Das bestrahlte CR39 wird eine längere Zeit (30 Minuten bis mehrere Stunden) bei einer konstanten Temperatur einer starken Lauge $(6 \mathrm{~m} \mathrm{KOH})$ ausgesetzt. An den Stellen, wo ein Ion aufgetroffen ist, ist die Ätzrate höher als in der Umgebung, so dass sich dort kegelförmige Vertiefungen im Material ergeben. Diese sind im Mikroskop sichtbar. Die Spurgröße hängt vom LET der Ionen, der Konzentration der Ätzflüssigkeit sowie der Ätztemperatur und -dauer ab [53]. Das Verfahren hat den Nachteil, dass die Messungen

\footnotetext{
${ }^{1}$ Track Analysis Systems Ltd., Bristol, UK.
} 
erst einige Stunden nach der Bestrahlung ausgewertet werden können und eignet sich daher nicht zur schnellen Optimierung des Strahls. Man kann aber die verschiedenen Strahlparameter bei Einzelteilchenexperimenten in Abhängigkeit von den optischen Einstellungen wie Magnetströmen oder Schlitzweiten im Nachhinein bestimmen. Außerdem kann über eine CR39 Bestrahlung die Zuverlässigkeit eines jeden Experiments mit Zellen überprüft werden.

Da sich mit CR39 jedes einzelne Ion mit hoher Ortsauflösung nachweisen lässt, kann man damit folgende Parameter bestimmen:

- Relative Strahlbreite

- Positioniergenauigkeit

- Anteil der Irrläufer (s. Abschnitt 6.4)

- Ionen Nachweiseffizienz

- Effizienz des beam shutters

\subsubsection{Silizium-Halbleiterzähler}

Mit einem Silizium-Halbleiterzähler kann man die Energie von Ionen mit hoher Genauigkeit bestimmen. Hierzu wird die Ladung gemessen, die ein Ion beim Durchgang durch das Detektormaterial durch Ionisierung erzeugt. Wenn die Tiefe des sensitiven Volumens größer als die Ionenreichweite ist, gibt das Ion seine komplette Energie ab, so dass die gemessene Ladung ein Maß für die Teilchenenergie ist. Da die Energie zur Erzeugung eines Elektron-Loch-Paares ca. 2,8 eV beträgt, und im Detektor keine weitere Signalverstärkung stattfindet, wird mit Silizium-Halbleiterzählern eine hohe Energieauflösung erreicht $(\approx 20 \mathrm{keV})$.

Die Detektion mit einem Halbleiterdetektor kann bei die Probe durchdringenden Teilchen zum Einzelteilchennachweis verwendet werden. Des Weiteren können einige andere Strahlparameter wie Teilchenrate und Energiebreite zu Testzwecken leicht überprüft werden. Ortsauflösende Messungen könnten durchgeführt werden, wenn man die Kante eines dünnen Streuers vor dem Detektor verfährt und aus den Spektren an verschiedenen Positionen das Strahlprofil bei geringen Teilchenraten rekonstruiert. 


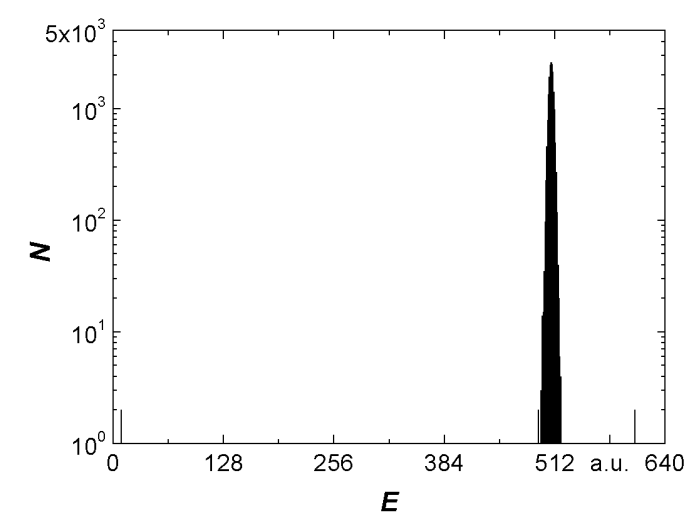

Abbildung 6.12: Energiespektrum von $3 \mathrm{MeV}$ Protonen.

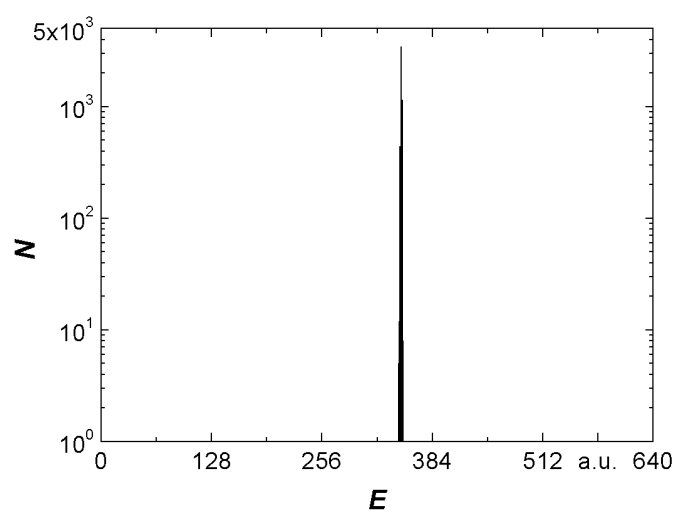

Abbildung 6.13: Energiespektrum von $20 \mathrm{MeV} \alpha$-Teilchen.

In den Abbildungen 6.12 und 6.13 sind zwei Energiespektren dargestellt. Die Energiebreite (einfache Standardabweichung) beträgt 2,7 bzw. 0,5 Kanäle. Sie ist bestimmt durch das Energiestraggling beim Durchtritt durch das Vakuumfenster. Dieses beträgt laut SRIM für $3 \mathrm{MeV}$ Protonen $22 \mathrm{keV}$ und für $20 \mathrm{MeV} \alpha$ Teilchen $33 \mathrm{keV}$. Die Breite der angepassten Normalverteilungen beträgt $14 \mathrm{keV}$ und $31 \mathrm{keV}$.

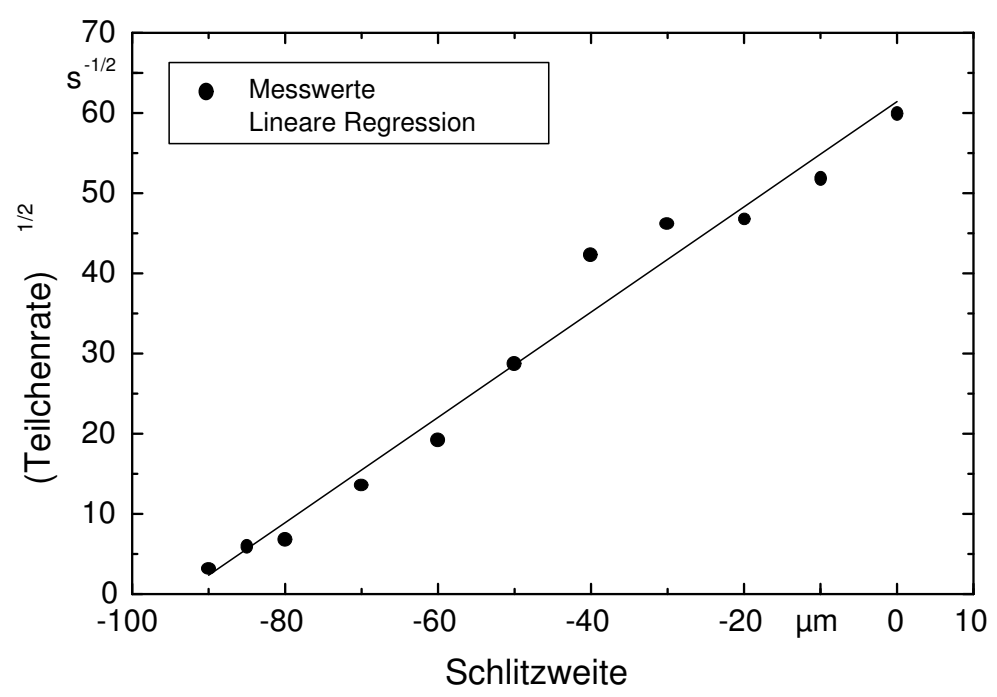

Abbildung 6.14: Teilchenrate in Abhängigkeit der Schlitzweite für $3 \mathrm{MeV}$ Protonen.

In Abbildung 6.14 ist die Abhängigkeit der Teilchenrate von der Öffnungsweite des Divergenzschlitzes angegeben. Aufgetragen ist hier die Wurzel der Zählrate, da der Divergenzschlitz in beiden Richtungen gleichzeitig variiert wurde. Es ist zu 
sehen, dass am PTB Mikro-Ionenstrahl Teilchenraten bis hinunter zu 10 Teilchen je Sekunde kontrolliert eingestellt werden können. Aus den Messungen lässt sich auch der tatsächliche Nullpunkt der Schlitzbreite bestimmen. Dieser liegt bei ca. -0,095 mm, da bei der optischen Einmessung der Schlitze ein minimaler Lichtspalt als null gesetzt wird.

Aus Abbildung 6.14 lässt sich zudem die Teilchendichte im Emittanzvolumen berechnen. Aus der Steigung der Regressionsgeraden von ergibt sich, dass eine Teilchenrate von $0,43 s^{-1} \mu m^{-2} \mu r a d^{-2}$ erwartet werden kann. Bei einem makroskopischen Emittanzvolumen von $5 \mathrm{~mm}$ mrad in beiden Richtungen ergäbe dies einen Strahlstrom von ca. 1,7 $\mu$ A. Dies ist in guter Übereinstimmung mit der Abschätzung aus Kapitel 3.2 .

\subsubsection{Szintillator und Photomultiplier}

Mit der dünnen Szintillatorfolie und dem PMT soll der Durchgang eines Ions festgestellt werden. Es gibt zwei Arten von Fehlern, die beide minimiert werden müssen:

wahr-negativ : Teilchendurchgang ohne Detektorsignal

falsch-positiv : Detektorsignal ohne Teilchendurchgang

Der Fehler der Art falsch-positiv wird durch die Stärke des Dunkelsignals bestimmt. Hierbei ist es wichtig, die Anzahl der Restlichtphotonen, die auf den PMT treffen, zu minimieren. Einen weiteren Beitrag liefern ionisierende Teilchen, die nicht aus dem primären Ionenstrahl stammen, sondern aus Radionukliden und kosmischer Strahlung. Bei optimalen Bedingungen ist der falsch-positiv Fehler durch die intrinsische Dunkelrate des Photomultipliers (s. Tabelle 5.3) gegeben. Diese bezeichnet die Signale am PMT, die durch spontane Emission von Elektronen aus dem Anodenmaterial oder den Dynoden, also ganz ohne auftreffende Photonen, entstehen.

Der Fehler der Art wahr-negativ wird im Wesentlichen durch die Größe des Detektorsignals bestimmt. Die Dicke der Szintillatorfolie und der Abstand zum Photomultiplier sind hier die entscheidenden Parameter. Als Teilchendurchgang werden solche Signale betrachtet, deren Größe über einer bestimmten Schwelle liegt. 
Ein weiterer Beitrag wird durch die Verzögerung des Deflektorsignals verursacht. Abhängig von der Teilchenrate können hierdurch zusätzliche, unerwünschte Ionen auf das Target gelangen, bevor der Deflektor den Ionenstrahl ausschaltet.

Beiden Arten von Fehlern kommt bei verschiedenen Experimenten durchaus unterschiedliche Bedeutung zu. Durch die Einstellung der Schwelle der Detektorelektronik ist es dem Experimentator möglich, das Verhältnis der Häufigkeiten der beiden Fehler gezielt einzustellen und den Gegebenheiten seines Experimentes anzupassen. Ein weiterer Parameter, der erheblichen Einfluss auf beide Arten von Fehlern hat, ist die Teilchenrate.

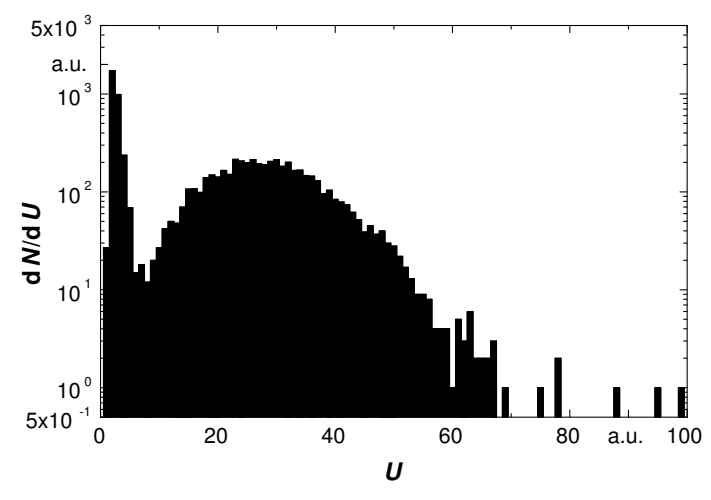

Abbildung 6.15: Impulshöhenspektrum von $20 \mathrm{MeV} \alpha$-Teilchen mit $10 \mu \mathrm{m}$ BC-400. U bezeichnet die Spannung am Signal-Ausgang des PMT.

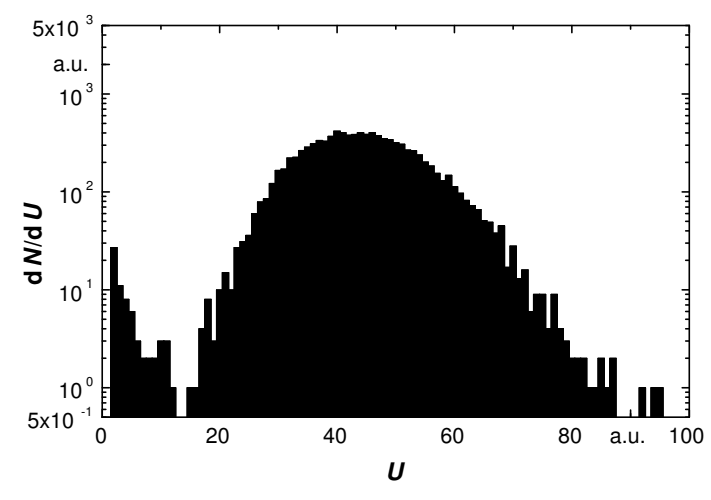

Abbildung 6.16: Impulshöhenspektrum von $20 \mathrm{MeV} \alpha$-Teilchen mit $25 \mu \mathrm{m}$ BC-400.

In den Abbildungen 6.15 und 6.16 sind zwei Impulshöhenspektren mit unterschiedlich dicken Szintillatorfolien dargestellt. Die beiden Spektren sind bei unterschiedlichen elektronischen Verstärkungen aufgenommen. In beiden Spektren ist das Signal des Restlichtes am linken Rand zu erkennen. Aufgrund der höheren Produktion von Szintillationsphotonen sind im zweiten Bild die Signale von Restlichtphotonen vollständig vom Signal der Ionen getrennt. Im ersten Bild beträgt der Gesamtfehler (wahr-negativ + falsch-positiv) ca. 1\%. Man beachte die logarithmische Skalierung.

Die Verschaltung der Detektorelektronik ist in Abbildung 6.17 schematisch dargestellt. Mit der Detektorelektronik werden die Teilchendurchgänge gezählt und vier verschiedene Spektren aufgenommen, die die Zuverlässigkeit jeder einzelnen Messung dokumentieren und eine Abschätzung für die oben erwähnten Fehler zulassen. 


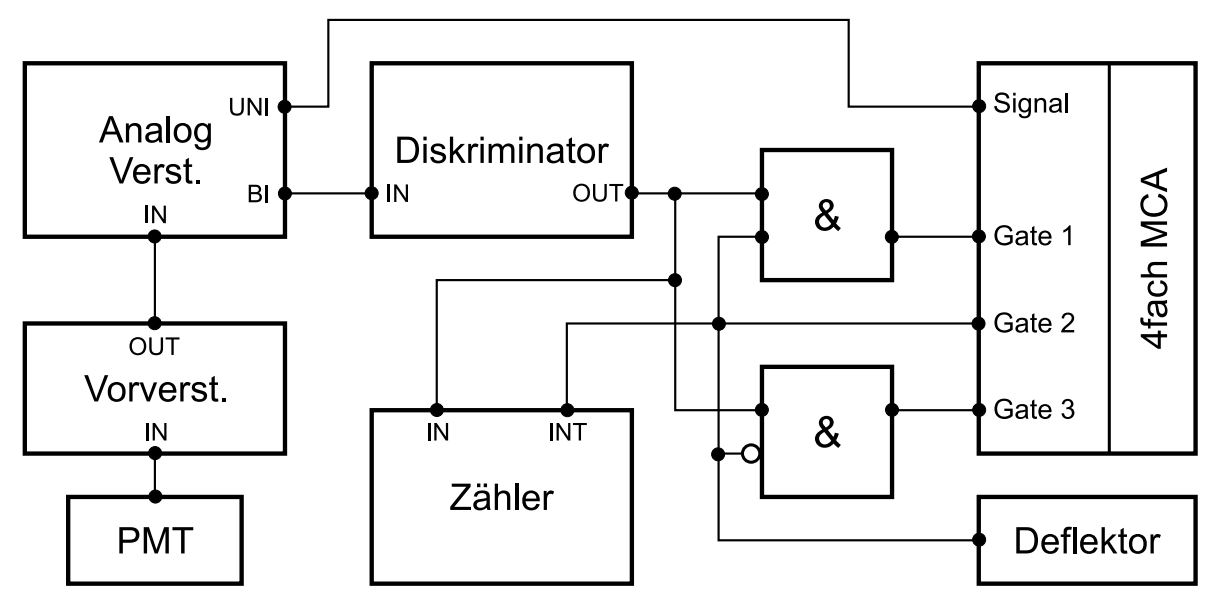

Abbildung 6.17: Logische Verschaltung der Detektorelektronik.

Spektrum 1 enthält die Signale, die über der Schwelle liegen und zum Zustand Strahl AN gehören (Abb.6.18). Dies sind die als Teilchendurchgänge betrachteten Signale. Spektrum 2 zeigt alle Signale, während des Zustandes Strahl AN (Abb. 6.19). Der Vergleich dieser beiden Spektren erlaubt die Kontrolle des Schwellenwertes des Diskriminators. Spektrum 3 beinhaltet alle Signale über der Schwelle während des Zustandes Strahl AUS (Abb. 6.20). Hiermit wird die Effizienz des Deflektors geprüft, und es kann die Rate der falsch-positiv Ereignisse abgeschätzt werden. Ein viertes Spektrum beinhaltet alle Signale, die während eines Bestrahlungsdurchgangs gemacht worden sind (Abb. 6.21). Da der Zustand Strahl AUS zeitlich bei weitem überwiegt (vgl. Kapitel 5.5), sind die Dunkelsignale in dem letzten Spektrum dominant.

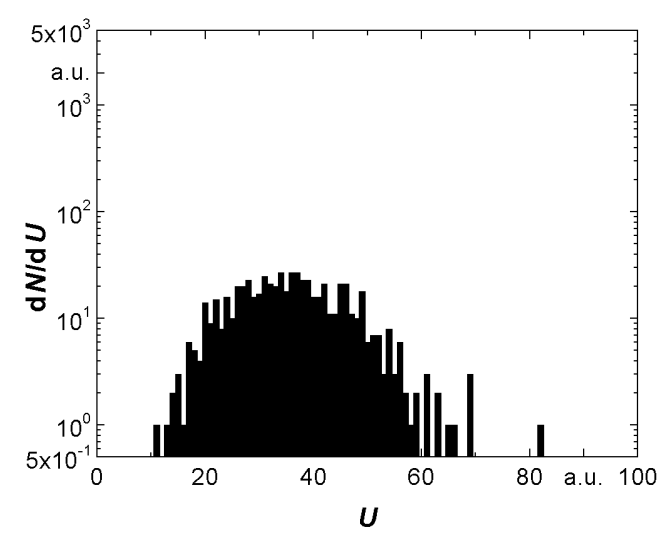

Abbildung 6.18: Spektrum 1: Teilchendurchgänge.

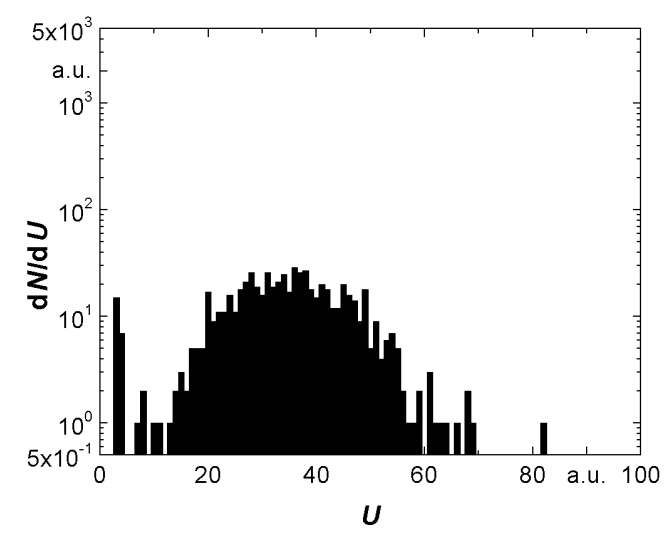

Abbildung 6.19: Spektrum 2: Alle Signale bei Strahl AN. 


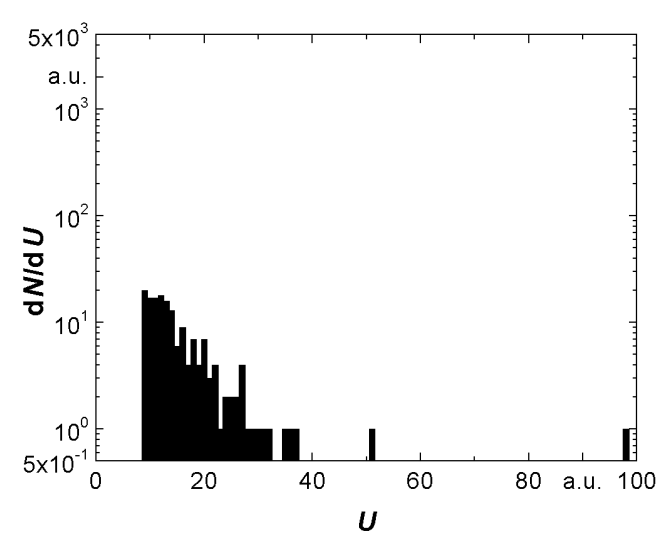

Abbildung 6.20: Spektrum 3: Signale oberhalb der Schwelle bei Strahl AUS.

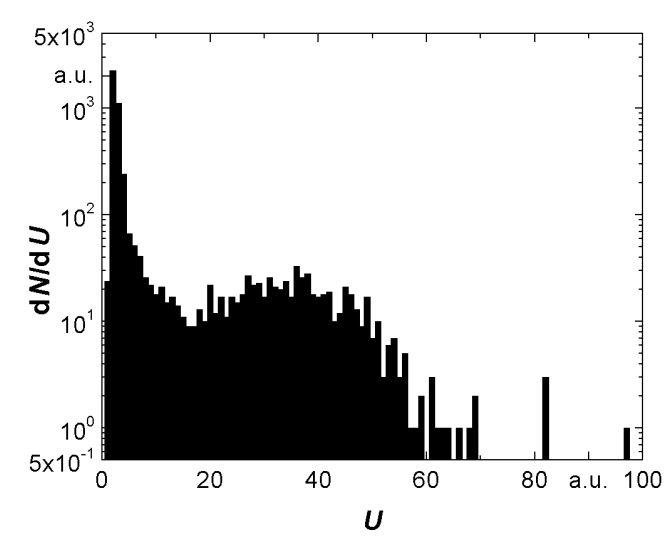

Abbildung 6.21: Spektrum 4: Alle Signale während der Bestrahlung.

Aus dem Verhältnis der Dunkelsignale in Spektrum 2 und 4 kann man den Anteil der tatsächlichen Bestrahlungszeit an der gesamten Messzeit berechnen. In diesem Fall beträgt er ca. 1,7 \%. Die Dunkelsignale aus Spektrum 3 werden nun mit diesem Faktor gewichtet. So erhält man einen Erwartungswert von 2,7 falschpositiv Signalen in der Messung. Bei insgesamt 615 Signalen entspricht das einem Anteil von 4,4\%o.

Der Anteil der wahr-negativ Ereignisse, die aus der Verzögerung des Deflektors entstehen, lässt sich leicht anhand der Teilchenrate berechnen. Bei 50 Teilchen je Sekunde und $10 \mu s$ Deflektorverzögerung beträgt er ca. 0,5\% \%, was hier einen Erwartungswert von 0,3 Teilchen ergibt. Falls es notwendig werden sollte, kann man diese Teilchen durch einen zusätzlichen Zähler messen. Der Anteil der wahrnegativ Ereignisse, die durch die Schwelle der Detektorelektronik entstehen, lässt sich durch eine Kurvenanpassung des Spektrums 1 an eine Poissonverteilung abschätzen. Die Berechnung ergibt, dass ca. 2\% der Intensität unterhalb der Schwelle liegt.

Die dargestellten Spektren stammen von einer CR39 Bestrahlung mit Protonen von $3 \mathrm{MeV}$ und einer Szintillatordicke von $25 \mu \mathrm{m}$. Diese Messungen sind in guter Übereinstimmung mit den Abschätzungen aus Abschnitt 5.2. Im Einzelfall kann die Nachweiswahrscheinlichkeit jedoch leicht variieren, da einige Parameter, wie z.B. PMT-Raumwinkel, Restlichtmenge und Teilchenrate nicht in jedem Experiment exakt gleich sind. 


\subsection{Ortsauflösung}

Die Strahlbreite ist gegeben durch die räumliche Verteilung der Strahlteilchen in der Ebene des Experiments. Ihre Minimierung ist ein wesentlicher Gegenstand der vorliegenden Arbeit. Die Strahlbreite kann auf verschiedene Weise gemessen werden, wobei jede Methode Vor- und Nachteile aufweist. Das zweidimensionale Strahlprofil, wie sie sich im Kamerabild darstellt, ist das Optimierungskriterium für die Einstellung der Strahlführung (vgl. Kapitel. 5.1).

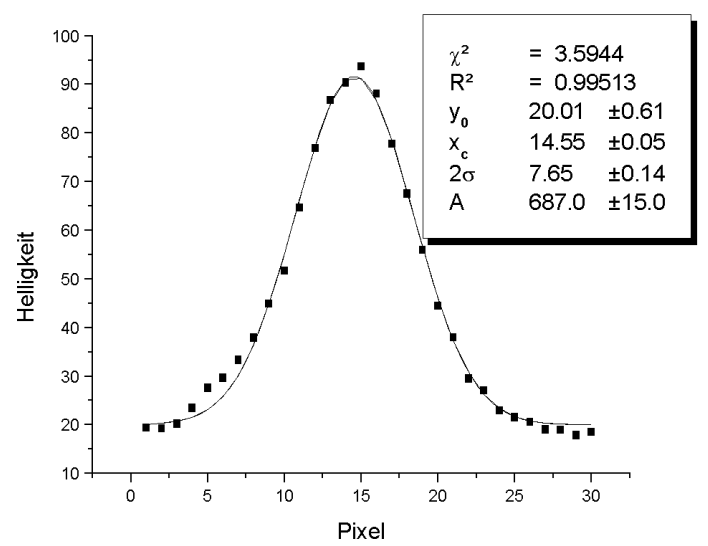

Abbildung 6.22: Horizontales Strahlprofil. Ein Pixel entspricht etwa $0,33 \mu \mathrm{m}$.

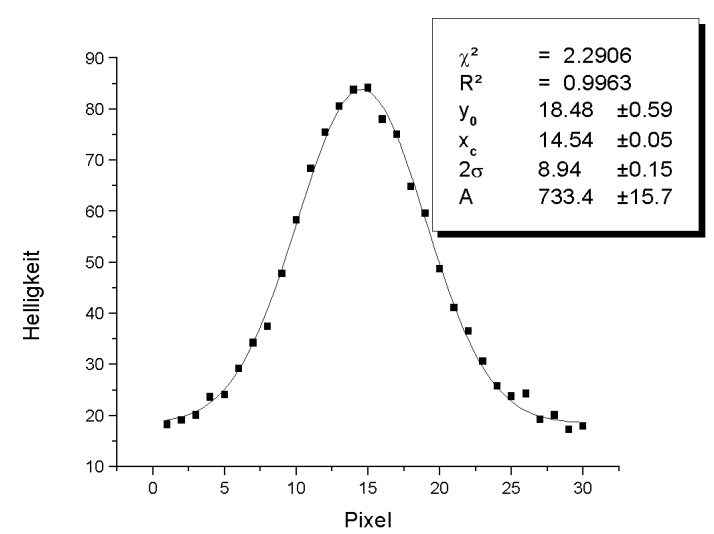

Abbildung 6.23: Vertikales Strahlprofil.

Während der Fokussierungsprozedur wird die Strahlbreite per visuellem Eindruck beurteilt. Man kann das Kamerabild aber auch numerisch auswerten. In den Abbildungen 6.22 und 6.23 sind das horizontale und das vertikale Intensitätsprofil des Strahls aus Abbildung 5.2 dargestellt.

An diese Profile kann man eine Gaußunktion

$$
y=y_{0}+\frac{A}{2 \sigma \sqrt{\pi / 2}} \exp \left(-\frac{2\left(x-x_{c}\right)^{2}}{(2 \sigma)^{2}}\right)
$$

anpassen.

Die Ergebnisse einer solchen Anpassung sind jeweils in der Legende der Abbildungen zu sehen. Die Strahlbreiten sind hier in Pixeln angegeben. In Länge umgerechnet betragen sie 1,2 $\mu \mathrm{m}$ bzw. 1,5 $\mu \mathrm{m}$ (einfache Standardabweichung).

Eine andere Methode zur Bestimmung der Strahlbreite ist die Bestrahlung von CR39. Diese Methode hat den Vorteil, dass sie bei Einzelteilchenexperimenten 


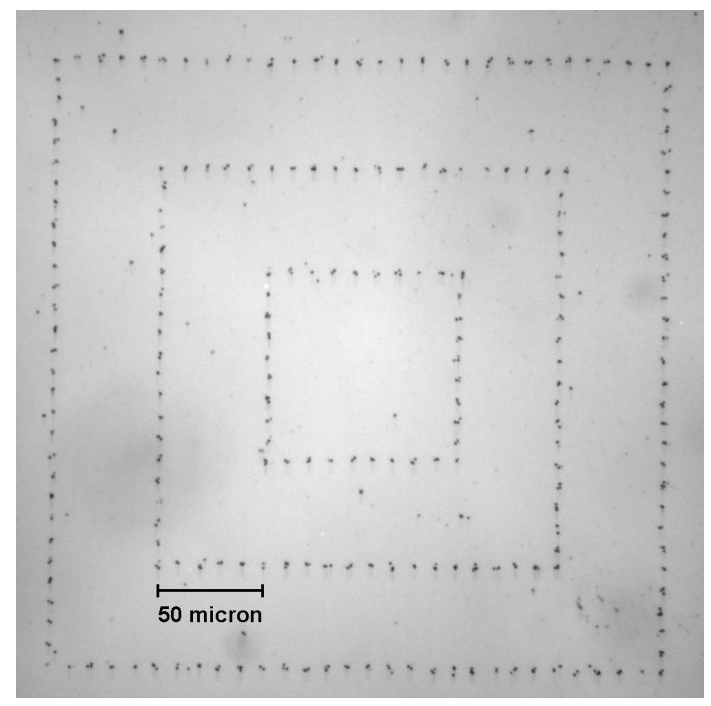

Abbildung 6.24: CR39 Muster von $20 \mathrm{MeV}$ $\alpha$-Teilchen. 3 Teilchen je Punkt (nominell).

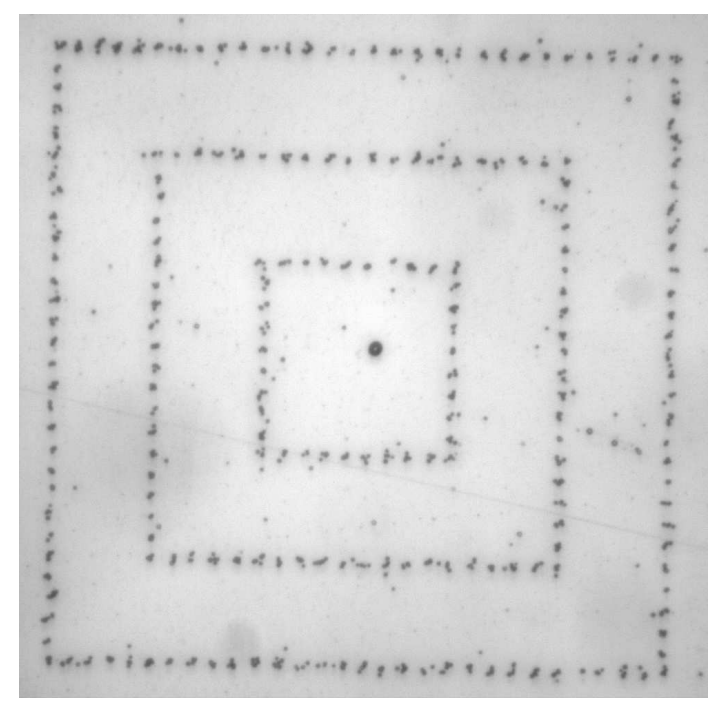

Abbildung 6.25: CR39 Muster von $3 \mathrm{MeV}$ Protonen. 5 Teilchen je Punkt (nominell).

angewandt werden kann. Es wurden eine Reihe von Bestrahlungen mit verschiedenen Mustern durchgeführt. In den Abbildungen 6.24 und 6.25 sind zwei solcher Muster dargestellt. Die Muster haben einen Punktabstand von jeweils $10 \mu \mathrm{m}$ und setzen sich aus zentrischen Quadraten der Kantenlängen $100 \mu \mathrm{m}, 200 \mu \mathrm{m}$ und $300 \mu m$ zusammen. Anhand der Linienbreiten kann man die horizontale und vertikale Strahlbreite abschätzen. In vielen Fällen sind die Spuren verschiedener Teilchen aufgrund räumlicher Überlagerung nicht zu trennen, so dass eine quantitative Analyse schwierig ist.

In Abbildung 6.26 ist ein Muster von $10 \times 10 \alpha$-Teilchen in einem Abstand von jeweils nominell $20 \mu \mathrm{m}$ dargestellt. Es sind folgende Unregelmäßigkeiten zu erkennen:

5 Bestrahlungspositionen sind leer

$\triangle 3$ Teilchen befinden sich auf falschen Positionen

1 Position ist mit 2 Teilchen besetzt

Wenn man diese fehlerhaft positionierten Teilchen außer Acht lässt, und die mittlere Abweichung der Teilchen von der Sollposition berechnet, erhält man Standardabweichungen von 1,0 $\mu \mathrm{m}$ und 0,9 $\mu \mathrm{m}$. Hierbei ist zu beachten, dass es keinen festen Bezugspunkt gibt und die Sollposition durch die mittlere Position der Teilchen berechnet wird, wobei die bekannten Pixeldimensionen und die bekannte 


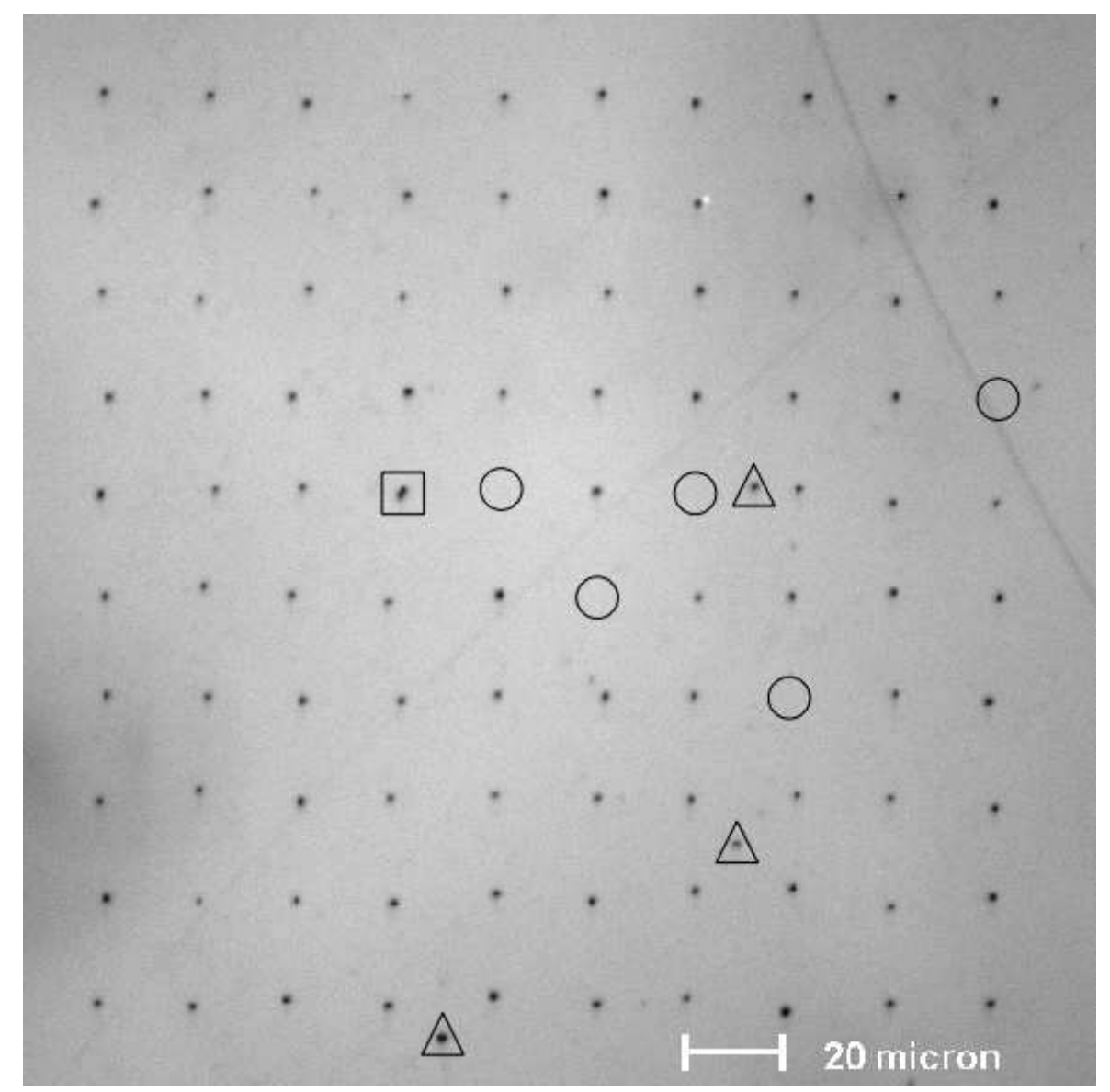

Abbildung 6.26: CR39 Muster von einzelnen $20 \mathrm{MeV} \alpha$-Teilchen.

Bildkippung mit berücksichtigt werden müssen. Bei dieser Berechnung geht man davon aus, dass die relative Positionierung durch die XY-Stage fehlerfrei ist. Da dies nicht der Fall ist (s. Abschnitt 5.6.1), ist die tatsächliche Strahlbreite geringer als der so berechnete Wert.

Ein weiterer Faktor ist die Konstanz der Position des Strahls. Da die Bestrahlung eines Zellträgers je nach Anzahl der Targets bis zu einer Stunde dauern kann, ist es wichtig, die Langzeitstabilität des Strahls zu untersuchen. Zudem werden bei einer Bestrahlung verschiedene mechanische Komponenten bewegt. Daher ist sicherzustellen, dass dies keinen Einfluss auf die Strahlposition hat. Bisher konnte eine gute Langzeitstabilität der Strahlfokussierung und der Strahlposition festgestellt werden. 


\title{
6.4 Irrläufer
}

Durch Messungen mit CR39 wurde festgestellt, dass bei Einzelteilchenexperimenten etwa $5 \%$ der Teilchen eine große Abweichung von der Sollposition aufweisen. Man kann den Anteil der Irrläufer ${ }^{2}$ messen, wenn man mit einer großen Anzahl Ionen eine Punktbestrahlung auf CR39 macht. Die Hauptintensität wird zu einem zentralen Fleck und die Streuteilchen in größerem Abstand davon lassen sich abzählen. Durch die Kenntnis der absoluten Anzahl über das PMT Signal, lässt sich der Streuanteil leicht bestimmen. In Abbildung 6.27 ist eine solche Messung dargestellt.

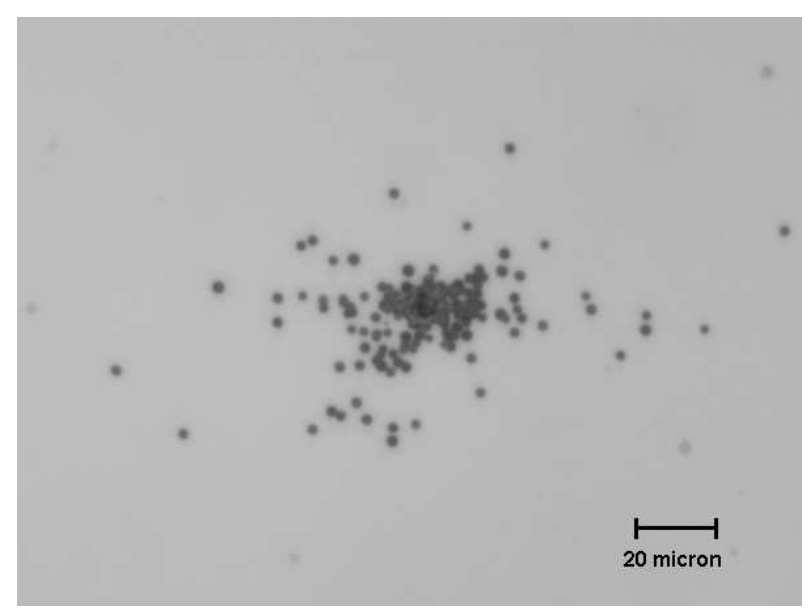

\begin{abstract}
Abbildung 6.27: Nachweis der Irrläufer mit dem CR39 Spurdetektor. Eine feste Position wurde mit 5000 Ionen bestrahlt. Nur die wenigen Teilchen außerhalb eines zentralen Kraters von ca. $5 \mu m$ Durchmesser sind als Einzelspuren sichtbar.
\end{abstract}

Die Irrläufer können verschiedene Ursachen haben:

- Streuung an einem der strahlbegrenzenden Schlitze

- Streuung am Restgas im Strahlrohr

- Löchrigkeit der Objektfolie oder unscharfe Begrenzung der Objektbohrung

- fehlerhafte Abbildung durch Aberrationen

Eine mögliche Ursache ist die Streuung am Energie-Ausgangschlitz (Schlitz 43). Hochenergetische Ionen können in das Schlitzmaterial eindringen und durch Vielfachstreuung abgelenkt werden, so dass sie es mit verringertem Impuls und geänderter Bahn wieder verlassen. Zur Verringerung dieses Effektes wurden unter

\footnotetext{
${ }^{2}$ Dieser Begriff wurde gewählt, um Verwechslungen mit dem in 4.1 erwähnten in der Aperturfolie gestreuten Strahl und mit den an der Fensterfolie gestreuten Ionen zu vermeiden.
} 
anderem spezielle Schlitzbacken vorgeschlagen [54]. An der PTB Anlage werden herkömmliche, rechteckige Wolframschlitzbacken von einem Millimeter Stärke verwendet, deren Oberfläche auf eine Rauhigkeit von weniger als einem Mikrometer geläppt wurde.

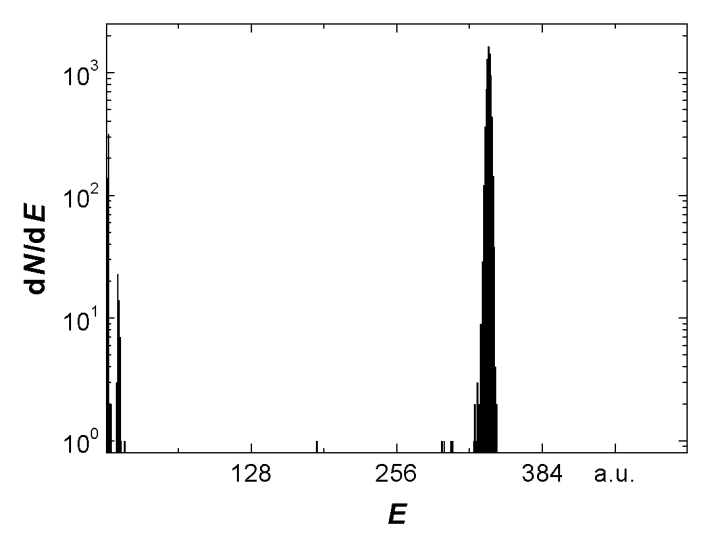

Abbildung 6.28: Energiespektrum von $3 \mathrm{MeV}$ Protonen bei einer Schlitzweite von $0,5 \mathrm{~mm}$, aufgenommen mit einem SiliziumHalbleiterdetektor..

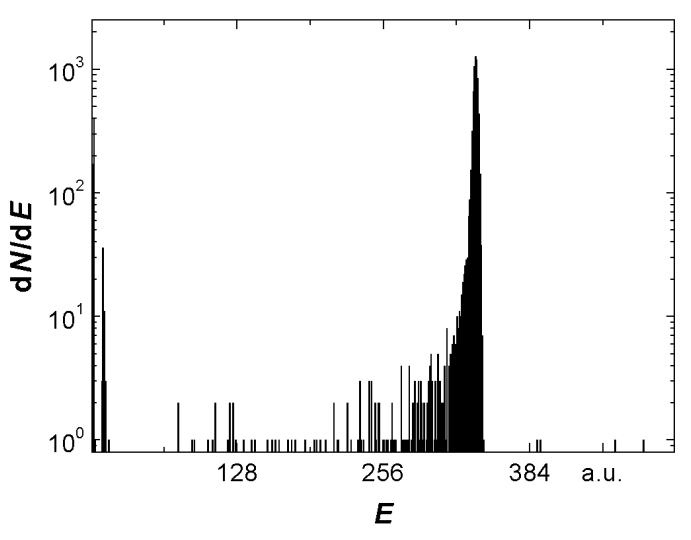

Abbildung 6.29: Energiespektrum von $3 \mathrm{MeV}$ Protonen bei einer minimalen Schlitzweite nahe $0,0 \mathrm{~mm}$.

Die Hypothese, dass bei verschiedenen Schlitzweiten ein unterschiedlicher Anteil von Irrläufern erzeugt wird, konnte experimentell bestätigt werden. Es wurden Messungen durchgeführt, bei denen das Energiespektrum der Teilchen unmittelbar hinter dem Analysiersystem in Abhängigkeit von der Schlitzweite aufgenommen wurde (Abb. 6.28 und 6.29). Dort ist der erhöhte Streuanteil bei geringer Schlitzweite deutlich sichtbar.

Aufgrund der Geometrie wurde bei diesen Messungen ein erheblicher Anteil der nicht vollständig im Schlitz gestoppten Ionen nachgewiesen. Die nachfolgenden ionenoptischen Elemente wie Anti-Streu-Schlitz, mikrofokussierendes Dublett und Vakuumfenster reduzieren diesen Anteil, und nur Irrläufer mit sehr geringer Abweichung werden durchgelassen. Diese geringen Abweichungen können aber durch die Abbildung der Quadrupole eine deutliche Ortsabweichung am Fokuspunkt bewirken.

Bei Messungen am Fokuspunkt konnte bisher keine Abhängigkeit des Irrläuferanteils von verschiedenen Schlitzeinstellungen beobachtet werden. Bei Messungen mit unterschiedlichen Schlitzweiten an Schlitz 43 wurde stets ein Anteil von $2 \%$ bis $5 \%$ von Irrläufern beobachtet. Zur endgültigen Klärung sind noch weitere Messungen notwendig. 


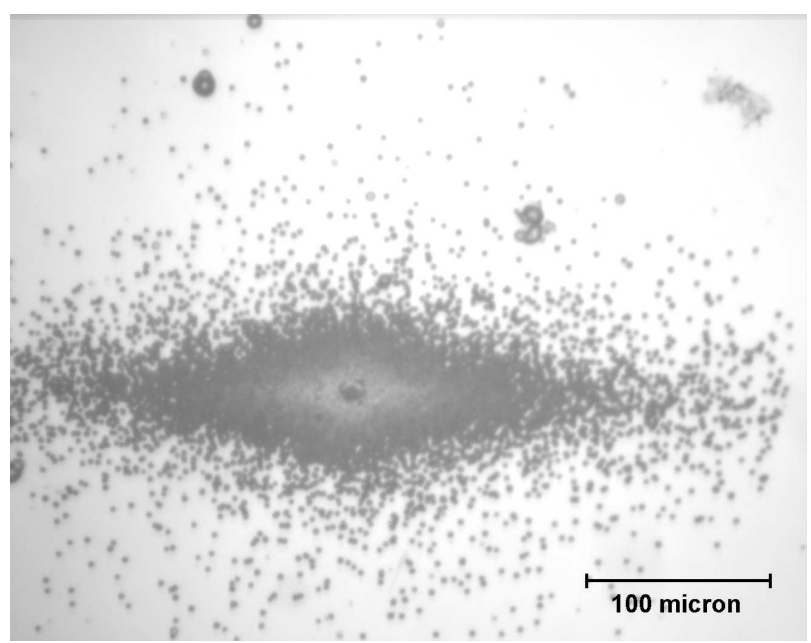

Abbildung 6.30: Bestrahlung von CR39 mit sehr hohen Intensitäten. Die Irrläufer sind auf eine Rautenfläche begrenzt.

Es wurde festgestellt, dass die Irrläufer räumlich nicht gleichverteilt oder normalverteilt auftreten, sondern auf eine Rautenfläche begrenzt sind (s. Abb. 6.30). Berechnungen legen nahe, dass die Raute ein ionenoptisches Abbild des AntiStreu-Schlitzes ist. Die Größe der Raute schließt Abbildungsfehler aus, selbst wenn man kombinierte Effekte von intrinsischen und parasitären Abberationen zugrunde legt, die bei der Berechnung der Strahloptik nicht berücksichtigt werden. Eine fehlerhafte Funktion der Objektblende konnte ebenfalls weitgehend ausgeschlossen werden, da der Irrläuferanteil bei der Titanfolie $(4 \mu \mathrm{m})$ der gleiche ist wie bei der Molybdänfolie $(5 \mu \mathrm{m})$ und zudem nicht von der Größe des Objektdurchmessers abhängt.

Die experimentellen Befunde stützen die Hypothese, dass die Irrläufer durch Streuung im Restgas verursacht werden. Wie die Abschätzung in Kapitel 3.3 zeigt, ist der Anteil der mit dem Restgas wechselwirkenden Ionen in der richtigen Größenordnung. Eine Berechnung des Effektes auf die optische Abbildung ist aber schwierig, da die Wirkung einer Streuung an einem Restgasmolekül von der Position im Strahlführungssystem abhängt.

Der Anteil der Irrläufer kann durch die Verwendung von Anti-Streu-Schlitzen verringert werden. Diese werden so eingestellt, dass sie den Soll-Strahl nicht einengen. Die Irrläufer, die ein viel größeres Phasenraumvolumen einnehmen, werden aber wirkungsvoll reduziert. Zur Zeit sind feste Anti-Streu-Schlitze mit einer Weite von $4 \mathrm{~mm}$ unmittelbar über dem zweiten Dublett installiert. Der Umbau dieser Schlitze ist zur Zeit in Arbeit (vgl. Abschnitt 7.2). 


\section{Kapitel 7}

\section{Zusammenfassung und Ausblick}

\subsection{Status der Anlage}

Die bisherigen Messungen zeigen, dass die Konzeption der Anlage und seiner Komponenten den experimentellen Anforderungen voll gerecht wird. Die Strahlführung erreicht die berechneten Abbildungseigenschaften. Die Korrektheit der ionenoptischen Berechnungen ist mithilfe des Folienstrahls in 1. Ordnung nachgewiesen worden. Das Verfahren, zur Strahljustierung den Folienstrahl zu verwenden, hat sich als praktikabel und sehr nützlich erwiesen. Die Schlitzstrommessungen dienen als wesentliche Hilfe bei der Justierung des Strahls. Die Diagnose der Abbildungseigenschaften mit dem YAG-Szintillators und der CCDKamera konnte bisher bei allen Randbedingungen problemlos durchgeführt werden.

Die angestrebten Leistungsmerkmale (s. Tabelle 1.2) konnten in allen wesentlichen Punkten erfüllt werden. Je nach Energie und Teilchensorte wird in Einzelteilchenexperimenten eine horizontale und vertikale Strahlbreite (Standardabweichung) zwischen $1 \mu \mathrm{m}$ und $2 \mu \mathrm{m}$ routinemäßig erreicht. Die Ortsgenauigkeit des Ionenstrahls (Strahlbreite und Konstanz der Strahlposition) ist somit für gezielte Zellkernbestrahlungen vollkommen ausreichend. Die Positionierung der Proben mithilfe des Mikropositioniertisches ist zur Zeit der limitierende Faktor in der Ortsauflösung. Ohne Korrekturen beträgt dessen Genauigkeit ca. $\pm 3 \mu \mathrm{m}$. Da der Mikropositioniertisch inzwischen kalibriert worden ist, können die Fehler in Zukunft korrigiert werden. Rechnerische Abschätzungen lassen erwarten, dass der Fehler dann auf $0,5 \mu m$ bis 1,0 $\mu m$ verringert werden kann. Eventuell muss ein Messsystem installiert werden, das die exakten Koordinaten während einer jeden Messung kontrolliert.

Der Nachweis einzelner Ionen mittels Szintillatorfolie und Photomultiplier funktioniert mit hoher Zuverlässigkeit. Bisher wurden für die experimentellen Tests 
vorwiegend $3 \mathrm{MeV}$ Protonen und $20 \mathrm{MeV} \alpha$-Teilchen verwendet, und hier stimmten die experimentellen Befunde gut mit den Vorhersagen für die Szintillationslichtausbeute und der resultierenden Detektionseffizienz sowie der Strahlaufweitung nach Austritt aus dem Vakuum überein. Daher kann für die anderen Teilchenenergien ebenfalls eine zuverlässige Vorhersagbarkeit der experimentellen Resultate erwartet werden. Durch die Aufnahme verschiedener Spektren während einer Bestrahlung ist es möglich, die Zuverlässigkeit jeder Messung abzuschätzen.

Die Häufigkeit der Irrläufer beeinträchtigt die guten Ergebnisse bei Ortsauflösung und Detektionseffizienz. Der Anteil der Teilchen, die eine große Abweichung von der Sollposition aufweisen und somit zu den falsch-positiv Ereignissen gezählt werden müssen, liegt derzeit bei $2 \%$ bis $5 \%$. Für viele radiobiologische Experimente ist das aber tolerierbar.

Die Fähigkeit, Einzelteilchenexperimente durchzuführen, kann als gegeben angesehen werden. Die Reduzierung des Strahlstroms auf wenige Ionen pro Sekunde kann kontrolliert erreicht werden, und mit dem Deflektor und der nachfolgenden ionenoptischen Abbildung wird ein vollständiges An- und Ausschalten des Strahls innerhalb von $10 \mu s$ erreicht.

Der PTB Mikro-Ionenstrahl stellt weltweit ein einzigartiges Instument dar. Im Gegensatz zu anderen existierenden Mikro-Ionenstrahlen zur radiobiologischen Forschung bietet er ein weiten Energiebereich von Ionen, der radiobiologische Untersuchung mit ganz unterschiedlichen Strahlenqualitäten (LET: $3 \ldots 200 \mathrm{keV} / \mu \mathrm{m}$ ) erlaubt. Durch die Fokussierung der Ionen wird erreicht, dass die Zellen einer nahezu monoenergetischen Strahlung ausgesetzt werden, während bei den kollimierten Mikrostrahlen immer ein Untergrund von niederenergetischen Ionen mit besonders hohem LET auftritt, der die Ergebnisse verfälschen kann, wenn geringe Effekte gemessen werden sollen. Das Prinzip der Fokussierung bietet zudem weitere Verbesserungsmöglichkeiten bezüglich der Erhöhung der Ortsauflösung und der Geschwindigkeit der Zellbestrahlungen.

\subsection{Apparative Verbesserungen}

Es hat sich gezeigt, dass mit den bisher zur Verfügung stehenden Mitteln die wesentlichen Zielvorgaben des Mikro-Ionenstrahls erreicht werden. Dennoch ist eine Verbesserung einzelner Elemente sowie die Installation zusätzlicher Komponenten wünschenswert. 
In den bisherigen Messungen weist der Mikro-Ionenstrahl größere Aberrationen auf als berechnet. Als Ursache hierfür wird eine mechanische Fehljustierung des horizontalen Quadrupoldubletts angesehen, dessen Achse nicht mit der Sollbahn übereinstimmt. Eine genauere Justierung als $\pm 1 \mathrm{~mm}$ war bisher nicht möglich. Es wurde daher ein neuer Justierrahmen konstruiert, der mithilfe von Kreuzrollenführungen und mechanischen Messuhren eine genaue und reproduzierbare Positionierung dieses Quadrupoldubletts mit einer Genauigkeit von besser als $50 \mu m$ erlaubt. Diese Komponente ist bereits installiert worden.

Die präzise Justierung des horizontalen Quadrupoldubletts verlangt nach eindeutigen Kriterien. Die Effekte auf den Ionenstrahl selbst, wie z.B. Größe der Aberrationen oder Positionsveränderung des Strahls bei Änderung der Quadrupolstärke (Ablenk-Effekt), können hier wichtige Hilfsmittel sein. Daher ist eine Messung der Strahllage und des Strahlprofils im horizontalen Teil der Strahlführung, z.B. bei Schlitz 42, sinnvoll. Möglicherweise würde es reichen, hier den Folienstrahl zu diagnostizieren. Ein Konzept für die Erweiterung der Strahldiagnose wird noch erarbeitet. Zu einer Verbesserung der Strahldiagnose könnte auch eine leichte Modifikation der Schlitze beitragen. Der isolierte Aufbau der Schlitzbacken und andere Instrumentierungsdurchführungen sollten in der Lage sein, die Zuverlässigkeit des Signals im Bereich unterhalb von $10 p A$ zu erhöhen. Eine Modifikation der Integratoren wird zudem den Messbereich der Elektronik nach oben bis ca. $1 \mu A$ ausdehnen.

In den Einzelteilchenexperimenten wurde bisher ein Anteil der Irrläufer von $2 \%$ bis $5 \%$ registriert. Deren Ursache ist zwar noch nicht geklärt, man kann aber annehmen, dass ihr Anteil sich durch die Installation von einstellbaren AntiStreu-Schlitzen reduzieren lässt. Bisher kommt ein mechanisch fixierter AntiStreu-Schlitz zum Einsatz, der eine Weite von $4 \mathrm{~mm}$ aufweist. Dieser schränkt im Vergleich zum Strahlrohrdurchmesser von $15 \mathrm{~mm}$ das verfügbare Emittanzvolumen ein, aber seine Weite wurde so gewählt, dass er auch für den Folienstrahl eine erhebliche Transmission zulässt. Seine wesentliche Funktion ist, die Einstellung der Korrekturmagnete zu ermöglichen, so dass der Strahl an der Stelle des mikrofokussierenden Dubletts eine zentrale Position hat. Bei Einzelteilchenexperimenten mit geringen Strahldivergenzen ist eine Verengung des Anti-Streu-Schlitzes auf $1 \mathrm{~mm}$ ohne Teilchenreduktion möglich. Deshalb wird der Anti-Streu-Schlitz in Zukunft ebenso wie die anderen Schlitze fahrbar sein. Unter der Annahme, dass die Irrläufer in der Nähe der Strahlachse gleichverteilt sind, ist ihre Anzahl proportional zu den Weiten in beiden Richtungen, und ließe sich somit um 
einen Faktor 16 verringern. Wenn sich die Streuung an Restgasmolekülen als die Ursache der Irrläufer herausstellen sollte, könnte ihr Anteil zudem durch eine Reduzierung des Restdruckes im Strahlführungssystem weiter verringert werden.

Der Einzelteilchennachweis mit der Szintillatorfolie liefert bereits zufriedenstellende Ergebnisse, aber apparative Modifikationen könnten in Zukunft die Fehler noch verringern. Zur Zeit wird mit einer recht einfachen Geometrie gearbeitet, in der ein einzelner Photomultiplier hinter der Probe angebracht ist. Eine Auslese des Lichtsignals durch die Kanten der Szintillatorfolie könnte das Verfahren auch für undurchsichtige Proben anwendbar machen. Eine Verwendung mehrerer Photomultiplier im Koinzidenzmodus oder die Verwendung von Photomultipliern mit Sammellinsen könnte die benötigte Szintillatordicke reduzieren. Zudem könnte durch zusätzliche Verdunkelungsmaßnahmen die Anzahl der Restlichtphotonen reduziert werden.

Ein wesentlicher Parameter bei Einzelteilchen-Experimenten, der bisher wenig erwähnt wurde, ist die Geschwindigkeit, mit der Zellen oder andere Targets bestrahlt werden können. Diese ist derzeit durch die mechanische Prozedur der Positionierung jedes einzelnen Targets auf ca. 20 Positionen je Minute beschränkt. Eine gezielte Ablenkung des Strahls könnte eine mechanische Positionierung ersetzen und zudem um ein Vielfaches schneller sein. Es ist hierbei aber zu berücksichtigen, dass die Dimensionen des Vakuumaustrittsfensters der Auslenkung des Strahls Grenzen setzen.

\subsection{Weitere Anwendungsgebiete}

Neben den radiobiologischen Anwendungen kann der Mikro-Ionenstrahl für eine Anzahl weiterer Experimente genutzt werden. Durch die einfache Positionierung der Targets und die Nutzung des externen Strahls (außerhalb des Vakuums) sowie die Möglichkeit, Strahlparameter wie Strahlstrom und Strahlenergie in einem weiten Bereich zu variieren, ist die Anlage recht flexibel. Teilweise wurden für die im Folgenden erwähnten Anwendungen schon Vorversuche durchgeführt.

Neben lebenden Zellen zeigen auch elektronische Bauteile eine Reaktion auf ionisierende Strahlung. Es ist beobachtet worden, dass einzelne Ionen zum Umkippen des Zustandes eines binären Speichers (single-event upset) und zum Versagen von Halbleiterelementen (single-event latch up) führen können [55, 56]. Beim Einsatz 
von Computern im Weltraum, wo diese einer erheblichen Strahlenbelastung ausgesetzt sind, stellen diese Effekte ein ernsthaftes Problem dar. Weitere Anwendungen in der Materialuntersuchung oder -modifikation sind denkbar [57].

Wie bereits in Kapitel 3 erwähnt, finden Ionenstrahlen seit vielen Jahren Anwendung in der Materialforschung. Der PTB Mikro-Ionenstrahl ist für diese Anwendung zwar nicht optimiert, ließe sie aber doch zu. Für Experimente, die einen hohen Strahlstrom erfordern, muss eine Verringerung der räumlichen Auflösung in Kauf genommen werden. Ein wichtiger Vorteil des PTB-Mikro-Ionenstrahls sind die hohen Strahlenergien, die die Analyse dickerer Proben zulassen. Bei diesen wird die Auflösung ohnehin durch Kleinwinkelstreuung im Probenmaterial beeinträchtigt.

Die Fähigkeit, abgezählte ionisierende Teilchen zur Verfügung zu stellen, kann auch für metrologische Zwecke verwendet werden. Man kann z.B. die mittlere Energie zur Herstellung eines Elektron-Ion Paares in einem Medium, den sogenannten W-Wert, mit hoher Genauigkeit bestimmen. Die W-Werte sind grundlegende Parameter, die für die Dosimetrie mit Ionisationskammern wichtig sind. Erste Versuche zur W-Wert Bestimmung konnten bereits durchgeführt werden. Auch für andere metrologische Fragestellungen könnte der Mikro-Ionenstrahl interessant sein, da einzelne Teilchen die kleinste Quantelung ionisierender Strahlung darstellen.

Ein weiteres Anwendungsgebiet, das bereits konkret geworden ist, ist die Aktivierung von Materialien zur Verschleißanalyse. Hierbei kann eine Probe mit dem Mikro-Ionenstrahl radioaktiv markiert werden. In Stahl können z.B. die Isotope ${ }^{56} \mathrm{Co}\left(T_{1 / 2}=77\right.$ Tage $)$ und ${ }^{57} \mathrm{Co}\left(T_{1 / 2}=272\right.$ Tage $)$ durch hochenergetische Protonen erzeugt werden. Durch die hohe Ortsauflösung können Bereiche von gewünschter Form und Größe aktiviert werden, und durch Energievariation kann die Tiefe der aktivierten Schicht eingestellt werden. Die Messung der Aktivität vor und nach mechanischer Beanspruchung ermöglicht dann die Quantifizierung des Abriebs an einem Probestück. Die Aktivität sollte gering gehalten werden, so dass die Strahlenbelastung unterhalb der gesetzlich zulässigen Grenzwerte liegt. Zur Aktivierung ist ein vergleichsweise hoher integrierter Strom notwendig $(\approx 1 \mu C)$. Bei geringer Ortsaufösung $(\approx 0,1 \mathrm{~mm})$ liegen die Bestrahlungszeiten aber in einem akzeptablen Bereich (Minuten bis Stunden).

Neben den genannten sind sicher noch eine Reihe weiterer Anwendungsgebiete denkbar. Nach der Elektronik sind zunehmend auch andere Technologien im 
Begriff in mikroskopische Bereiche vorzudringen, wie z.B. die Mechanik und die Sensorik. 


\section{Literaturverzeichnis}

[1] International Commission On Radiation Units and Measurements Radiation Quantities and Units

ICRU report 33, (1980)

[2] International Commission On Radiation Units and Measurements The Quality Factor in Radiation Protection

ICRU report 40, (1986)

[3] International Commission On Radiation Units and Measurements Linear Energy Transfer ICRU report 16, (1970)

[4] W. R. Leo

Techniques for Nuclear and Particle Physics Experiments Springer-Verlag, Berlin 1994

[5] E.R. Bartels, D. Harder The Microdosimetric Regulations of Nanometric Regions Radiation Protection Dosimetry, Vol. 31 No. 1/4 pp. 211 -215 (1990)

[6] Bundesumwelministerium Strahlenschutzverordnung http://www.bmu.de/download/dateien/strahlenschutzvo.pdf

[7] Roesch, W.C. (Hrsg.)

US-Japan Joint Reassessment of Atomic Bomb Radiation Dosimetry in Hiroshima and Nagasaki - Final Report.

Radiation Effects Research Foundation (RERF), 1987

[8] Ellet W.H., Samet, J.M.

Epidemiological studies on uranium miners and other groups exposed to radon

In: Low Dose Radiation: Biological Bases of Risk Estimation. Editors: K.F. Baverstock \& J.W. Stather

Taylor \& Francis, London 1989

[9] D.A. Pierce and M. Vaeth

Cancer Risk Estimation from A-Bomb Survivors: Extrapolation to Low Doses, Use of Relative Risk Models and Other Uncertainties 
In: Low Dose Radiation: Biological Bases of Risk Estimation. Editors: K.F. Baverstock \& J.W. Stather

Taylor \& Francis, London 1989

[10] Rossi H.H., Kellerer A.M.

The Validity of Risk Estimates of Leukemia Incidence Based on Japanese Data

Radiation Research 58, 131-140 (1974).

[11] Bundesamt für Strahlenschutz

Umweltradioaktivität in der Bundesrepublik Deutschland 1996 und 1997 Daten und Bewertung -

BfS-SCHR-21/00

[12] Bundesamt für Strahlenschutz

1. Bericht des Strahlenschutzregisters des BfS mit Daten des ÜberwachungSjahrs 1998

BfS-ISH-188/00

[13] C.R. Geard et. al.

Microbeam mediated cellular effects: single $\alpha$ Particle induced chromosomal damage, cell cycle delay, mutation and oncogenic transformation

Microdosimetry, An Interdisciplinary Approach, Edited by D.T. Goodhead, P. O’Neal, H. Menzel (1997)

[14] Li-Jun Wu et al.

Targetted Cytoplasmic Irradiation with Alpha Particles induces Mutations in Mammalian Cells

Proc. Natl. Acad. Sci. USA, Vol. 96, pp. 4959 - 4964, April 1999, Cell Biology

[15] Sheldon Wolff et al.

The adaptive response of human lymphocytes to very low dose of ionising radiation: A case of induced chromosomal repair with the induction of specific proteins.

In: Low Dose Radiation: Biological Bases of Risk Estimation. Editors: K.F. Baverstock \& J.W. Stather

Taylor \& Francis, London 1989

[16] S.G. Sawant et al.

The Bystander Effect in Radiation Oncogenesis: I. Transformation in C3H 
$10 \mathrm{T1} / 2$ Cells In Vitro can be Initiated in the Unirradiated Neighbors of Irradiated Cells

Radiation Research 155, 397 - 401 (2001)

[17] K. Prise et al.

Studies of bystander effects in human fibroblasts using a charged particle microbeam

Int. Journal of Radiation Biology 1998, Vol.74, 387-395

[18] H. Magasawa and J.Little

Induction of Sister Chromatid Exchanges by Extremely Low Doses of $\alpha-$ Particles

Cancer Research 52, 6394-6396, (1992)

[19] ICRP Veröffentlichungen 60

Empfehlungen der Internationalen Strahlenschutzkommission 1990

Bundesamt für Strahlenschutz der Bundesrepublik Deutschland (Hrsg.)

Gustav Fischer Verlag, Stuttgart - Jena - New York, 1993

[20] H.J. Brede et al.

The Braunschweig Accelerator Facility for Fast Neutron Research. I. Building Design and Accelerator

Nucl. Instr. and Meth. 69 (1980) 349-358

[21] R.E. Zirkle \& W. Bloom

Irradiation of Parts of Individual Cells

Science, 117, 487-493, 1953

[22] L.Braby

Microdosimetry and Microbeam Irradiation

Microdosimetry, An Interdisciplinary Approach, Edited by D.T. Goodhead, P. O’Neal, H. Menzel (1997)

[23] C.R. Geard et. al.

Single-particle irradiation of mammalian cells at the Radiobiological Research Accelerator Facility: induction of chromosomal changes

Nucl. Inst. Meth. Phys. Res. B 54, 411-416 (1991).

[24] M. Folkard et al.

A charged-particle microbeam: I. Development of an experimental system 
for targetting cells individually with counted particles.

Int. Journal of Radiation Biology 1997, Vol.72, No. 4, 375-385

[25] M. Folkard et al.

A charged-particle microbeam: II. A single-particle micro-collimation and detection system.

Int. Journal of Radiation Biology 1997, Vol.72, No. 4, 387-395

[26] Diverse Autoren

5th International Workshop on Microbeam Probes Of Cellular Radiation Response. May 26 - 27, 2001, Stresa, Italy.

Programme and Abstracts. (werden in Radiation Research veröffentlicht)

[27] K.L. Brown

A First- and Second-Order Matrix Theory for the Design of Beam Transport Systems and Charged Particle Spectrometers

SLAC Report-75

[28] F. Hinterberger

Physik der Teilchenbeschleuniger

Springer Verlag, Berlin Heidelberg New York 1997

[29] K. Wille

Physik der Teilchenbeschleuniger und Synchrotronstrahlungsquellen

B.G. Teubner, Stuttgart 1992

[30] G.J.F. Legge

A History of Ion Microbeams

Nucl. Inst. Meth. Phys. Res. B 130, 9 (1997).

[31] G.W. Grime and F. Watt

Beam Optics Of Quadrupole Probe Forming Systems

A. Hilger Ltd., Bristol 1984

[32] G.W. Grime and F. Watt (Editors)

Principles and Applications of High-Energy Ion Microbeams

A. Hilger Ltd., Bristol 1987

[33] K.L: Malmquist

Analytical Techniques in Nuclear Microprobes

Nucl. Inst. Meth. Phys. Res. B 104, 138 (1995). 
[34] S. Ya. Yavor et al.

Achromatic Quadrupole Lenses

Nucl. Instr. and Meth. 26 (1964) 13-17

[35] F. Kohlrausch

Praktische Physik

VEB Deutscher Verlag der Wissenschaften, Berlin 1962

[36] Christen, Meyer

Grundlagen der allgemeinen und anorganischen Chemie

Salle und Sauerländer, Frankfurt (Main), 1997

[37] K.L. Brown, D.C. Carey, Ch. Iselin and F. Rothacker

TRANSPORT A Computer Program for Designing Charged Particle Beam Transport Systems

CERN 80-04

[38] D.C. Carey, K.L. Brown, Ch. Iselin TURTLE With MAD Input (Trace Unlimited Rays Through Lumped Elements) A Computer Program for Simulating Charged Particle Beam Transport Systems and DECAY TURTLE Including Decay Calculations Fermilab-Pub-99/232 (1999)

[39] U. Dötsch and A. D. Wieck Nanodevices produced with focused ion beams. Nucl. Inst. Meth. Phys. Res. B 139, 12 (1998).

[40] Particle Data Group Particle Properties Data Booklet 1992

[41] J.F. Ziegler

The Stopping and Range of Ions in Matter; Instruction Manual www.research.ibm.com/ionbeams/SRIM/SRIMA.HTM

[42] Burr-Brown IC Data Book, Linear Products Burr-Brown Int. GmbH, 1995

[43] M. Moszyński et al. Properties of the YAG:Ce scintillator Nucl. Instr. and Meth. A, 345 (1994) 461-467 
[44] Diagnostics Instruments, Inc.

Spot Camera Product Information

www.diaginc.com

[45] Hamamatsu Photonics

Photomultiplier Tubes and Assemblies

Product Catalog 1998

[46] Hamamatsu Photonics

Metal Package Photomultiplier Tube R7400U Series

Product Infomation Sheet 1999

[47] Bicron, Saint-Gobain Industrial Ceramics, Inc.

Organic Scintillators, Related Materials and Detectors

Product Catalog 1997

[48] R. Madey et al.

The Response of NE-228A, NE-228, NE-224 and NE-102 Scintillators to Protons from 2.43 to $19.55 \mathrm{MeV}$.

Nucl. Instr. and Meth. 151 (1978) 445-450

[49] G. Dietze und H. Klein

NRESP4 and NEFF4 Monte Carlo Codes for the Calculation of Neutron Response Functions and Detection Efficiencies for NE-213 Scintillation Detectors

PTB Bericht, PTB-ND-22, (1982)

[50] R. L. Craun and D. L. Smith Madey et al.

Analysis of Response Data for Several Organic Scintillators

Nucl. Instr. and Meth. 80 (1970) 239-244

[51] Fa. Märzhäuser Wetzlar

Produkt Katalog 1999 / 2000

[52] B.G. Cartwright et al.

A Nuclear Track Recording Polymer of Unique Sensitivity and Resolution Nucl. Instr. and Meth. 153 (1978) 457-460

[53] M. Luszik-Bhadra et al.

Microdosimetric Investigations in a Proton Therapy Beam with Sequentially 
Etched CR-39 Track Detectors

Radiation Protection Dosimetry Vol. 66 Nos. 1-4, pp. 353-358 (1996)

[54] R. Nobiling et al.

Collimation of Ion Beams to Micrometer Dimensions

Nucl. Inst. Meth. 130, 325-334 (1975)

[55] B.E. Fischer et al.

An example what you can miss in single-event-effect testing, when you do not have a microprobe

Nucl. Inst. Meth. Phys. Res. B 158, 245-249 (1999).

[56] K.M. Horn et al.

Nuclear Microprobe Imaging of Single-Event Upsets

IEEE Tr.NS Vol.39, No.1, (1992)

[57] B.E. Fischer and S. Metzger

Single-Ion Micromechanics

MRS Bulletin, Vol.25, No.2, (2000), Materials Research Society 


\section{Danksagung}

Diese Arbeit, bzw das, was sie beschreibt, ist selbstverständlich nicht das Werk eines einzelnen, sondern konnte nur entstehen, weil viele Menschen mit Ihren Kenntnissen und Fähigkeiten dazu beigetragen haben. Bei einigen von Ihnen möchte ich mich an dieser Stelle bedanken.

Bei Herrn Prof. Dr. Frankenberg und Herrn Dr. Brede, die das Projekt PTBMikro-Ionenstrahl initiiert haben, bedanke ich mich für die interessante Aufgabenstellung und die gute und freundliche Zusammenarbeit, mit der sie mich stets unterstützt und ermutigt haben, ohne mich in meinen Freiheiten einzuschränken.

Ich danke Herrn Prof. Dr. Schmahl von der Georg-August-Universität Göttingen dafür, dass er die Aufgabe übernommen hat, diese Arbeit zu betreuen und als Referent zu begutachten.

Herrn Prof. Dr. D. Harder, der als Kurator der PTB das Projekt zudem auf politischer Ebene unterstützt hat, verdanke ich einige wichtige Anregungen und viele fruchtbare Diskussionen insbesondere zum Thema Strahloptik.

Herrn Prof. Dr. W. Wieck von der Universität Bochum danke ich für das kleine aber wesentliche Detail, dass er an seinem fokussierten Ionenstrahl die Objektblenden in völlig unkomplizierter Kooperation hergestellt hat

Außerdem danke ich Prof. Dr. B. Michael und Dr. M. Folkard vom Gray Cancer Institute in London, die mir mit einem dreiwöchigen Aufenthalt an ihrem Institut Gelegenheit gegeben haben, ihren Mikrostrahl kennenzulernen und von ihren Erfahrungen zu profitieren.

Da es sich in diesem Fall um eine experimentelle Arbeit handelt, bei der eine große Anzahl Komponenten entwickelt oder installiert wurde, schulde ich insbesondere den technischen Mitarbeitern des Fachlaboratoriums Neutronenmetrologie der PTB großen Dank.

Von diesen möchte ich Wolfgang Beverung besonders hervorheben, der als Betriebsingenieur nicht nur für den Beschleunigerbetrieb insgesamt verantwortlich ist, sondern auch viele wertvolle Beiträge bei den elektronischen Komponenten und bei deren Computeransteuerung geliefert hat. 
Den Beschleuniger-Operateuren Oliver Döhr, Helmut Eggestein, Thomas Heldt und Manfred Hoffmann gilt ebenfalls mein großer Dank. Sie haben ihren Sachverstand und ihre Erfahrungen an vielen Stellen mit großem Engagement eingebracht und waren dabei auch immer offen und flexibel, neue Herausforderungen anzunehmen und eingetretene Pfade zu verlassen.

Für die Realisierung der mechanischen Elemente bedanke ich mich bei den Mitarbeitern der Werkstatt des Labors, insbesondere bei Andreas Eckert und Dietrich Pink. Zudem gilt mein Dank den Mitarbeitern der PTB-Feinmechanikwerkstatt.

Für die Gestaltung vieler Grafiken und Kurven danke ich Helga Beldy und Elke Heinemann.

Außerdem möchte ich unserer Verwaltungsangestellten Brigitte Heise danken, die es geschafft hat, dass ich in drei Jahren PTB nicht ein einziges Formular korrekt ausfüllen musste.

Außer den genannten haben mich noch viele andere Mitarbeiter im Fachloboratorium und in der PTB in meiner Arbeit unterstützt. Auch ihnen gilt mein Dank.

Diese Arbeit wurde durch die Europäische Komission finanziell unterstützt. 


\title{
Lebenslauf
}

\author{
Name: \\ Klaus-Dieter Greif \\ Geburtsdatum: \\ 15.11.1967 \\ Geburtsort: \\ Dinslaken \\ Familienstand: \\ Ledig \\ Staatsangehörigkeit: \\ Deutsch \\ Eltern: \\ Agnes Greif, geb. Rohling, Kinderkrankenschwester \\ Dieter Greif, Maurer \\ Geschwister: \\ Annette Greif, 36 Jahre \\ Thomas Greif, 33 Jahre

$\begin{array}{lll}1974- & -1978 & \text { Grundschule Hünxe } \\ 1978- & -1987 & \text { Gymnasium Voerde / Abitur } \\ 08 / 87- & 03 / 89 & \text { Zivildienst im Pflegebereich, Ev. Krankenhaus Wesel } \\ 04 / 89- & 07 / 89 & \text { Weiterbeschäftigung als Pfleger, Ev. Krankenhaus Wesel } \\ 08 / 89-\quad 09 / 89 & \text { Studienvorbereitendes Praktikum: } \\ & & \text { Altbausanierung in Berlin, Fa. Bauer \& Melchert, Voerde } \\ 10 / 89-09 / 90 & \text { Studium der Architektur, Universität Dortmund } \\ \text { ab 10/90 } & & \text { Studium der Physik (Diplom), Universität Dortmund } \\ 10 / 92 & & \text { Vordiplom } \\ 03 / 96 & & \text { Diplom-Physiker-Hauptprüfung } \\ 04 / 96-03 / 97 & \text { Diplomarbeit: Aufbau und Test eines Profilmonitors } \\ & & \text { für den DELTA-Linearbeschleuniger am Institut } \\ & \text { für Beschleunigerphysik und Synchrotronstrahlung } \\ & \text { der Universität Dortmund } \\ 03 / 97 & \text { Abschluss des Studiums mit Diplom } & \text { Arbeitssuchend } \\ 04 / 97-05 / 98 & \text { Beschäftigt an der PTB, Entwicklung und Erprobung } \\ \text { seit 06/98 } & \text { des PTB-Mikro-Ionenstrahls }\end{array}$

